\title{
Experimental and Theoretical Study of The Electronic Structure of Single-crystal $\mathrm{BaBiO}_{3}$

\author{
by
}

Shadi Balandeh

B.Sc., Sharif University of Technology, 2011

M.Sc., The University of British Columbia, 2013

A THESIS SUBMITTED IN PARTIAL FULFILLMENT OF

THE REQUIREMENTS FOR THE DEGREE OF

DOCTOR OF PHILOSOPHY

in

The Faculty of Graduate and Postdoctoral Studies

(Physics)

THE UNIVERSITY OF BRITISH COLUMBIA

(Vancouver)

August 2018

(c) Shadi Balandeh 2018 
The following individuals certify that they have read, and recommend to the Faculty of Graduate and Postdoctoral Studies for acceptance, the dissertation entitled:

Experimental and Theoretical Study of The Electronic Structure of Single-crystal $\mathrm{BaBiO}_{3}$

submitted by Shadi Balandeh in partial fulfillment of the requirements for the degree of Doctor of Philosophy in Physics.

\section{Examining Committee:}

Supervisor: Prof. George A. Sawatzky

Supervisory Committee Member: Prof. Doug Bonn

Supervisory Committee Member: Prof. Mona Berciu

Supervisory Committee Member: Prof. Mark Van Raamsdonk

University Examiner: Prof. Srikantha Phani

University Examiner: Prof. Sarah Burke 


\section{Abstract}

Hole doped bismuth perovskite is one of the rare examples of a threedimensional high transition temperature superconducting oxide $\left(T_{c}=34\right.$ $\mathrm{K})$ without a transition metal cation. The undoped compound, $\mathrm{BaBiO}_{3}$, also shows closely interlinked electronic and structural phase transitions and a controversial insulating mechanism. Understanding the electronic structure of the parent compound, $\mathrm{BaBiO}_{3}$, can give valuable insight into both its superconducting mechanism, in particular, and into the physics of the perovskites family, in general.

In this work, we first grow high-quality single crystals of $\mathrm{BaBiO}_{3}$ by congruent melting technique and characterize the crystals with x-ray diffraction, $\mathrm{x}$-ray photoemission, and transport properties measurements.

We then investigate the electronic structure of the material from both theoretical and experimental perspectives. Experimentally, we study the crystals through x-ray absorption, x-ray emission, and photoelectron spectroscopies. X-ray spectroscopy verifies the results of density functional theory (DFT) regarding the overall band structure featuring strong $\mathrm{O} 2 p$ character of the empty anti-bonding combination of the hybridized $\mathrm{Bi} 6 s$ and O $2 p$ states. We also develop a routine to successfully clean the sample's surface in order to achieve the intrinsic O $1 s$ x-ray photoemission spectrum.

Finally, we employ the "exact diagonalization single cluster configuration interaction" method to investigate the ground state electronic structure of the material and to understand the measured $\mathrm{O} 1 s$ x-ray photoemission result. From the analysis of the core level line shapes, we conclude that the dominant $\mathrm{O} 2 p$-Bi $6 s$ hybridization energy scale determines the low energy scale electronic structure. We also find that for a wide range of electronic parameters, the holes reside primarily on the ligand oxygen anions rather than the bismuth cation consistent with the band structure calculation but contrary to what is traditionally assumed for this material. This analysis provides further insight into the importance of self-doped oxygen $2 p$ states in this high $T_{c}$ family of oxides. 


\section{Lay Summary}

In this work, we study the electronic structure of $\mathrm{BaBiO}_{3}$ which is the parent compound of a family of materials with unusual physical properties including superconductivity. To study this, high-quality single crystals are required the growth of which was the first task of this project. We then used several $\mathrm{x}$-ray spectroscopy techniques sensitive to the electronic structure and used a model calculation to interpret the experimental findings.

It is generally assumed that in $\mathrm{BaBiO}_{3}$ the oxygen does not play a prominent role in determining the physical properties of the material such as electrical conductivity and magnetic susceptibility. However, we find evidence to show otherwise. We show it is, in fact, the oxygen electronic states that dictate these interesting physical and electronic behaviours. 


\section{Preface}

This thesis is mainly based on one published paper [Experimental and theoretical study of the electronic structure of single-crystal $\mathrm{BaBiO}_{3}[1]$ by Shadi Balandeh, Robert J. Green, Kateryna Foyevtsova, Shun Chi, Oleksandr Foyevtsov, Fengmiao Li, and George A. Sawatzky, Phys. Rev. B 96, 165127 - Published 13 October 2017 ] for which I was responsible for all major areas of crystal growth, data collection, analysis, creating the plots and manuscript composition under supervision and guidance of my supervisor, Prof. Sawatzky, and with assistance from the other co-authors. Dr. Robert Green, in particular, played an essential role in aiding me in doing the experiments at the Canadian Light Source (CLS) and also in the use of the Quanty program for the computational results of the chapter 5.

For this work, I have carried out the experiments partly at the Stewart Blusson Quantum Matter Institute at the University of British Columbia and partly at the Canadian Light Source (CLS) research facility. In chapter 2 , the reciprocal map x-ray diffraction and resistivity measurements have been carried out by Dr. Oleksandr Foyevtsov. In chapter 3 , the Density Functional Calculations have been done by Dr. Kateryna Foyevtsova. The rest of the experiments and calculations have been done by me. In chapter 5, the computations have been done via Quanty program developed by M. Haverkort et al. 


\section{Table of Contents}

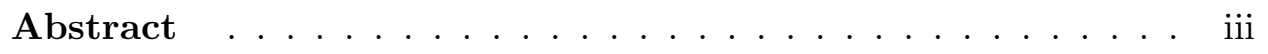

Lay Summary ...................... iv

Preface $\ldots \ldots \ldots \ldots \ldots \ldots \ldots \ldots \ldots$

Table of Contents .................. . . .

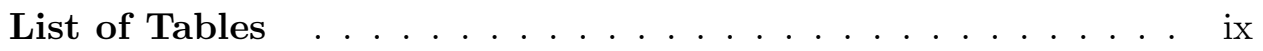

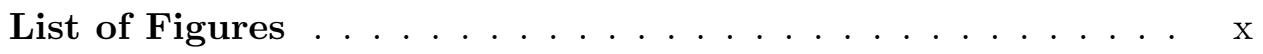

Acknowledgements ....................... xvi

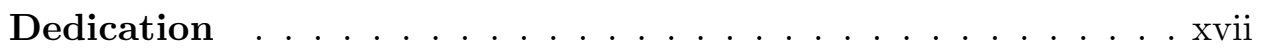

1 Bismuthates ...................... 1

1.1 Electronic structure ................ 1

1.1.1 Bismuthates and Nickelates .......... 5

1.2 Crystal Structure . . . . . . . . . . . . . 7

1.3 Superconductivity . . . . . . . . . . . . 8

1.3.1 Bismuthates and Cuprates .......... 9

$2 \mathrm{BaBiO}_{3}$ Single Crystal Growth $\ldots \ldots \ldots_{12}$

2.1 Introduction . . . . . . . . . . . . . . 12

2.2 Single Crystal Growth Procedure . . . . . . . . . . 15

2.3 Characterization . . . . . . . . . . . . . . . 27 27

2.3.1 X-ray Photoemission Spectroscopy . . . . . . . . 27

2.3 .2 X Ray Diffraction ... . . . . . . . . . 33

2.3 .3 Resistivity . . . . . . . . . . . . . 42

2.4 Conclusion . . . . . . . . . . . . . . . . 44 


\section{X-ray Absorption and Resonant X-ray Emission Spectroscopy} 45

3.1 Introduction . . . . . . . . . . . . . . 45

3.1.1 Theory ....................... 47

3.1.2 Detection Techniques ............. 51

3.1.3 REIXS Beam-line at CLS . . . . . . . . . . 55

3.2 Oxygen K-edge XAS Results . . . . . . . . . . . . . . 55

3.3 Oxygen K-edge RXES Results . . . . . . . . . . . . . 59

3.4 Theoretical Interpretation of The Experiment . . . . . . . 60

3.5 Conclusion . . . . . . . . . . . . . . 66

4 X-ray Photoemission . . . . . . . . . . . . . . 6 67

4.1 Introduction . . . . . . . . . . . . . . . 67

4.1.1 XPS detection technique . . . . . . . . . 70

4.1 .2 Theory . . . . . . . . . . . . . 70

4.1.3 XPS, core levels and satellites . . . . . . . . 72

4.2 XPS Results . . . . . . . . . . . . . . . 773

4.3 Conclusion . . . . . . . . . . . . . . . 80

5 Exact Diagonalization Cluster Model . . . . . . . . . 81

5.1 Introduction . . . . . . . . . . . . . . . . . . 81

5.1 .1 Atomic Model . . . . . . . . . . . . . 82

5.1 .2 Crystal Field Model . . . . . . . . . . . . . . 84

5.1 .3 Ligand Field Model . . . . . . . . . . . . . . . 88

5.2 Computations . . . . . . . . . . . . . . . . 91

5.2 .1 Cluster ...................... . . . 91

5.2 .2 Model Hamiltonian . . . . . . . . . . . . . . 91

5.3 Ground-State Spin Calculation . . . . . . . . . . . . 96

5.4 Hole Distribution Calculation . . . . . . . . . . . . . 97

5.5 XPS calculation . . . . . . . . . . . . . . . . 100

5.6 Conclusion . . . . . . . . . . . . . . . . . . 103

6 Summary and Future Work . . . . . . . . . . . . . 104

6.1 Summary . . . . . . . . . . . . . . . . . . 104

6.2 Future Works . . . . . . . . . . . . . . . . . 105

6.2.1 Further Characterization of the Single Crystals . . . 105

6.2.2 Electron Doped Material . . . . . . . . . . . 105

6.2.3 Resonant Photoemission Measurements . . . . . . 106

6.2.4 Pair Distribution Function Measurements . . . . . . . 106 
Table of Contents

Bibliography . . . . . . . . . . . . . . . 107

Appendices

A $\mathrm{BaBiO}_{3}$ Bragg Reflections $\ldots \ldots \ldots_{117}$ 


\section{List of Tables}

$2.1 \mathrm{BaBiO}_{3}$ lattice parameters in $I 2 / m$ symmetry taken from Ref. [2] and used to simulate the powder diffraction pattern in Fig.

$2.7 \ldots \ldots \ldots \ldots \ldots \ldots \ldots \ldots$

2.2 The atomic ratios of the grown single crystal given by the XPS measurement (Fig. 2.14 ). The accuracy of the results derived from this technique (described in the text) is within

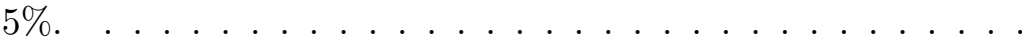

$2.3 \mathrm{BaBiO}_{3}$ single crystal lattice parameters measured at temperatures $90 \mathrm{~K}$ and $210 \mathrm{~K}$. . . . . . . . . . . . . . 35

2.4 The $\mathrm{R}$ value for different Bragg reflection pairs. $\mathrm{R}$ is defined in Eq. 2.19 and is indicative of contribution from different crystal domains. This result implies that the given crystal was $13 \%$ twinned. . . . . . . . . . . . . .

4.1 The relation between quantum numbers, atomic notation and $\mathrm{x}$-ray notation for the first few energy levels [3. $j$ is the total angular momentum quantum number which takes $|\ell-s| \leq$ $j \leq|\ell+s|$ values where $s=1 / 2$ for electrons. . . . . . . . 69 


\section{List of Figures}

1.1 Top: Density of states of a compressed and expanded $\mathrm{BiO}_{6}$ octahedron projected on to the $\mathrm{Bi}-6 s$ orbital and combinations of the $\mathrm{O}-p \sigma$ orbitals for $\mathrm{SrBiO}_{3}$ structure from Ref. [4]'s DFT(LDA) calculations [4, 5]. Bottom: The octahedron with O- $p \sigma$ orbitals at the corners hybridized through nearest-neighbor hopping integrals of $-t$ and the corresponding eigenstates in the table for different symmetries [4] . . .

$1.2 \mathrm{DFT}(\mathrm{LDA})$ band structures of $\mathrm{BaBiO}_{3}$ and $\mathrm{SrBiO}_{3}$ calculated with a supercell of dimensions $\sqrt{2} a \times \sqrt{2} a \times \sqrt{2} a$. The band structures are almost identical around the Fermi energy except for a larger gap in $\mathrm{SrBiO}_{3}$ due to its more prominent structural distortions. The Fermi energy is set to zero [4]. . .

1.3 a: Mott-Hubbard insulators where the gap is determined by the Coulomb energy $U$ versus c: charge-transfer insulators where the gap is determined by $\Delta$ which is smaller than $U$. b: charge fluctuations corresponding to the energies $\Delta$ and $U$. The blue and red orbitals represent the ligand oxygen $p$, and the transition metal $d$ orbitals, respectively $[5,6]$. . . .

1.4 The schematic illustration of the perovskite nickelate (on the left) and bismuthate (on the right) electronic structures. In the bismuthate, the deep Bi- $6 s$ states with the $A_{1 g}$ symmetry hybridize strongly with the combination of the $\mathrm{O}_{2} p$ states with the same symmetry. Consequently, the state of $A_{1 g}$ symmetry and mainly $\mathrm{O}_{2} p$ character are pushed above the Fermi level. In the nickelate, the situation is similar but the cation states are the Ni-3d states with the $E_{g}$ symmetry which again mix with the $\mathrm{O}_{2} p$ states with the same symmetry [5]. . . . 
1.5 The crystal structure of perovskite $\mathrm{BaBiO}_{3}$. The green balls at the corners of the octahedra represent the oxygen anions. The black and white balls at the centers of the octahedra represent the bismuth cations in expanded and collapsed octahedra, respectively [4]. . . . . . . . . . . . . 7

1.6 Summary of $\mathrm{Ba}_{x} \mathrm{~K}_{1-x} \mathrm{BiO}_{3}$ properties taken from Ref. [7]. . 9

1.7 The phase diagrams of top: $\mathrm{Ba}_{1-x} \mathrm{~K}_{x} \mathrm{BiO}_{3}$ and bottom: $\mathrm{BaBi}_{1-x} \mathrm{~Pb}_{x} \mathrm{O}_{3}$ compounds. Due to the lack of phase purity for $\mathrm{BaBi}_{1-x} \mathrm{~Pb}_{x} \mathrm{O}_{3}$ samples and hence the non-conclusive results the $\mathrm{BaBi}_{1-x} \mathrm{~Pb}_{x} \mathrm{O}_{3}$ phase diagram is perhaps only qualitatively correct [7]. . . . 10

2.1 An overview of the most used crystal-growth techniques $[8$. These techniques fall into four main categories: solid-state growth, vapour phase growth, melt growth and solution growth. In this work a combination of solid-state+melt growth was ultimately used to grow $\mathrm{BaBiO}_{3}$ crystals. . . . . . . . . 14

2.2 Temperature-steps for the $\mathrm{BaBiO}_{3}$ single crystal growth as given by Ref. [9]. (The highest temperature of $1010^{\circ} \mathrm{C}$ in the plot has to be an error. Based on the text and the $\mathrm{BaBiO}_{3}$ melting temperature of $1040^{\circ} \mathrm{C}$, it has to be $1100^{\circ} \mathrm{C}$.) . . . 17

2.3 First growth attempt and its Laue-gram. The result was not single crystal. (unsuccessful) . . . . . . . . . . 18

2.4 The construction of the furnace (top) with the insulating ceramic disk (bottom) to introduce a temperature gradient along the crucible for the second attempt. . . . . . . . . 19

2.5 Second growth attempt and its Laue-gram. Here the previous procedure was repeated but a temperature gradient was also introduced along the crucible. The result was not single crystal. (unsuccessful) . . . . . . . . . . . . .

2.6 Third growth attempt and its Laue-gram. Here the previous procedure was repeated but the calcined powders was ground and homogenized before the melt. The result was golden brown as expected for $\mathrm{BaBiO}_{3}$ but not single crystal. (unsuccessful) ........................ 21

2.7 Simulated (top) and measured (bottom) $\mathrm{BaBiO}_{3}$ powder diffraction patterns with $\mathrm{Cu}-\mathrm{K} \alpha \mathrm{X}$-ray source. . . . . . . . 2 23

2.8 The thermal process for the $\mathrm{BaBiO}_{3}$ singe crystal melting growth. The red arrow indicates the slow cooling step from above the melting point to the below. . . . . . . . 24 
2.9 Fourth growth attempt and its Laue-gram. The melting procedure was performed on $\mathrm{BaBiO}_{3}$ powder which had been synthesized in advance through solid-state reaction. The result was $1 \times 1 \mathrm{~mm}^{2}$ plates of $\mathrm{BaBiO}_{3}$ single crystals. ( successful). 25

2.10 Fifth (final) growth attempt. Increasing the melt surface area by using a wider crucible ( $25 \mathrm{~mm}$ OD) resulted in growing bigger single crystals. (successful) . . . . . . . . . .

2.11 X-ray back-reflection Laue-gram of the grown crystal with the $\mathrm{x}$-rays oriented along the [100] crystallographic direction in a cubic unit cell. . . . . . . . . . . . . . .

2.12 A carbon $1 s$ x-ray photoemission spectrum with the background signal corrected using the top: linear and bottom: Shirley algorithms. The figures are taken from Ref. [10] and they have been modified slightly by adding the labels. . . . .

2.13 Omicron Multi-probe MXPS system at the Surface Science Facility of the REIXS beam-line at the Canadian Light Source (CLS) . . . . . . . . . . . . . . .

2.14 X-ray photoemission spectrum of $\mathrm{BaBiO}_{3}$ single crystal at

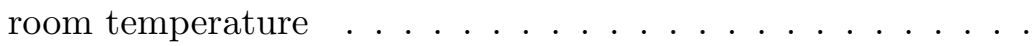

2.15 A schematic representation of a two-dimensional crystal lattice. An incident beam hitting the crystal undergoes a constructive (in phase) diffraction when the path difference is equal to an integral multiple of the wavelength: $n \lambda=2 d \sin \theta$. (Bragg's law) . . . . . . . . . . . . . 33

2.16 X-Ray Diffraction pattern on a grown $\mathrm{BaBiO}_{3}$ single crystal with the "as grown" surface facing. . . . . . . . . . 36

2.17 Schematic illustration of the coplanar diffraction geometry. $n$ is the normal to the sample surface and $n_{\phi}$ is the normal to the diffracting lattice planes. $\alpha_{i}$ and $\alpha_{f}$ are the angles of incident and exit. $\omega$ is the angle between the incident beam and the diffracting lattice planes, $\omega=\alpha_{i}+\phi . \quad \ldots \ldots \ldots$. . .

2.18 Reciprocal space maps of crystal $\mathrm{BaBiO}_{3}$ top: around (110) and (002) and bottom: around (220) and (004) Bragg diffractions. The axes are in reciprocal lattice unit (r.l.u) which is $\frac{\lambda}{2 d_{h k l}}$ where $\lambda=1.54059 \AA . \quad \ldots \ldots \ldots \ldots \ldots$. . . . . . . .

2.19 Reciprocal space maps of crystal $\mathrm{BaBiO}_{3}$ top: around (330) and (006) and bottom: around (440) and (008) Bragg diffractions. The axes are in reciprocal lattice unit (r.l.u) which is $\frac{\lambda}{2 d_{h k l}}$ where $\lambda=1.54059 \AA . \quad \ldots \ldots \ldots \ldots 1$ 
2.20 Schematic representation of the 4-probe resistivity measurement using a lock-in amplifier. . . . . . . . . . . . . 43

2.21 Resistivity measurement of $\mathrm{BaBiO}_{3}$ crystal. An abrupt change in slope, i.e. activation energy $\left(E_{a}\right)$, happens at $T=233 \mathrm{~K}$.

3.1 The three main regions of an x-ray absorption spectrum [11]. 47

3.2 The schematic representation of $\mathrm{x}$-ray photoemission/absorption, normal and resonant emission processes. $[12] \ldots \ldots$. . . . 52

3.3 Some standard XAS detection techniques. The blue regions qualitatively represent the detection depths of each technique.

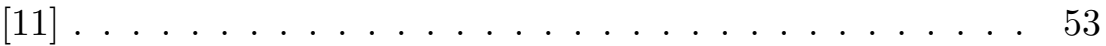

3.4 XAS machine at the REIXS end-station at CLS [13]. . . . . 55

3.5 Some specifications of the REIXS beam-line end-station at CLS [14]. . . . . . . . . . . . . . . . . . . 55

3.6 Oxygen K-edge XAS of single-crystal $\mathrm{BaBiO}_{3}$ top: measured at $20 \mathrm{~K}$ and $400 \mathrm{~K}$ with vertical polarization. bottom: with polarizations perpendicular (vertical) and parallel (horizontal) to the sample surface plane at room temperature. No significant temperature or polarization dependence is observed. 57

3.7 The reproducibility of the top: TEY and bottom: TFY signals are tested for the oxygen K-edge XAS of single-crystal $\mathrm{BaBiO}_{3}$. The measurements are repeated after 24 hours while sample was kept in vacuum. The TFY is found reproducible over time unlike the TEY. . . . . . . . . . . . . .

3.8 Main: oxygen K-edge resonant x-ray emission spectra on a single crystal of $\mathrm{BaBiO}_{3}$ at three different Resonant Incident Energies (RIE). Inset: oxygen K-edge XAS spectrum. The red dots show the resonant incident energies used for the RXES measurement. . . . . . . . . . . . . . .

3.9 Oxygen K-edge RXES and XAS spectra measured on a $\mathrm{BaBiO}_{3}$ single crystal and the DFT(LDA) oxygen $p$ projected density of states of $\mathrm{BaBiO}_{3}$. The energy has been shifted by -528.5 $\mathrm{eV}$ for the experimental axis to facilitate comparison with the calculation. . . . . . . . . . . . .

3.10 Oxygen K-edge XAS compared to oxygen $p$ projected density of unoccupied states from top: DFT(LDA). middle: DFT(LDA) and the XSPEC package which takes into account the transition probabilities in calculating the intensity. bottom: DFT (LDA) on a $2 \times 2 \times 2$ supercell with a core-hole. . . . . . 65 65 
4.1 XPS spectrum of lead illustrating schematically how exciting electrons from each orbital can give rise to a distinct peak in the spectrum [3]. Those electrons that are excited and leave the surface without an energy loss produce the characteristic peaks and those that undergo inelastic scattering and energy loss processes contribute to the background. . . . . . . 68

4.2 Schematic presentation of the core $1 s$ XPS process . . . . . 69

4.3 This shows how at a low electron take off angle (grazing) the surface sensitivity is enhanced and the thin oxide layer on the silicon is more dominant [15]. . . . . . . . . . . . 71

4.4 Core $1 s$ XPS spectra of elements with the atomic numbers of 3 to 9 . The binding energy increases with increasing the atomic number $[16,17] \ldots \ldots \ldots \ldots$

4.5 Oxygen $1 s$ XPS of $\mathrm{O}_{2}$ and $\mathrm{H}_{2} \mathrm{O}$ in their gas phase. $\mathrm{O}_{2}$ is paramagnetic with two unpaired electrons in its valence shell. The core-hole created through the XPS process can interact with these and gives two non-degenerate states observed as the two XPS lines. $\mathrm{H}_{2} \mathrm{O}$ is diamagnetic with all its valence electrons paired; therefore, it gives one single XPS peak. [17, 18] 74

4.6 Oxygen 1s and carbon 1s XPS spectra after our first attempt in cleaning the surface of the single crystal $\mathrm{BaBiO}_{3}$. . . . 76

4.7 Oxygen $1 s$ and carbon $1 s$ XPS spectra at different stages of our cleaning procedure on the single crystal $\mathrm{BaBiO}_{3}$. The removal of the carbon peak confirms the effectiveness of the procedure. . . . . . . . . . . . . .

4.8 Oxygen $1 s$ and carbon $1 s$ XPS spectra of single crystal $\mathrm{BaBiO}_{3}$ after the optimized cleaning procedure described in the text. The oxygen spectrum loses most of its structure with the progression of the cleaning but remains slightly asymmetric. . . .

$5.1 d$ orbitals in various crystal field symmetries. The specific symmetry of the ligands affect the energy levels of the metal ion $[1] . \ldots \ldots \ldots \ldots \ldots \ldots \ldots$

5.2 The arrangement of $d$ electrons in the ground state of an octahedral complex following the Hund's rules. $N$ is the number of distinct pairs of electrons with parallel spins. The crystal field potential here is the energy difference between the $t_{2 g}$ and the $e_{1 g}$ orbitals. . . . . . . . . . . 87 
5.3 A schematic representation of a: $\sigma$ and b: $\pi$ bonding and antibonding molecular orbitals of $p$ orbitals. The figure is taken from Ref. [19] and has been modified slightly by adding the signs. . . . . . . . . . . . . . . . . 90

5.4 The model single cluster of $\mathrm{BaBiO}_{3}$ with six oxygens at the corners and a bismuth atom at the center. . . . . . . 92

5.5 A schematic presentation of the involved hybridization terms in our model Hamiltonian. There are bismuth $s$ orbital at the centre and oxygen $p$ orbitals at the corners of the octahedron. a: $t_{O_{p}-B i_{6 s}}=\sqrt{6} t_{s p \sigma}$. b: $\sigma$ and $\pi$ oxygen $p$ hopping terms. c (and a): $t_{p p}=1 / 2\left(t_{p p \sigma}-t_{p p \pi}\right) . \mathrm{d}: t_{p p}^{\prime}=1 / 2\left(t_{p p \sigma}+t_{p p \pi}\right)[1] . \quad$. 94

5.6 The total spin of the ground state as a function of crystal field energy $\left(E_{C F}\right)$ and oxygen-bismuth hybridization energy $\left(t_{s p \sigma}\right)$ for $U=3 \mathrm{eV}$. The first arrow indicates that we need to turn on the $E_{C F}$, so that for $t_{s p \sigma}=0$ we get the correct triplet ground state for a bismuthless octahedron with two holes. The second arrow shows we have to increase the $t_{s p \sigma}$ energy so that we achieve the expected low spin ground state when the bismuth is present. . . . . . . . . . .

5.7 The average number of holes as a function of $a$ : $\mathrm{Bi} 6 s / \mathrm{O} p$ hybridization energy $\left(t_{s p \sigma}\right)$ for $\Delta=-4 \mathrm{eV}$. and $b$ : the charge transfer energy $\Delta$ for $t_{s p \sigma}=2.1 \mathrm{eV}$. For a large range of parameters, holes reside primarily on the ligand oxygens. . .

5.8 Calculated oxygen $1 s$ XPS spectra with all the hybridization terms set to zero for different core-hole potential $(Q)$ values. The curves are shifted along the "Intensity" axis. . . . . . . 101

5.9 Calculated oxygen $1 s$ XPS spectra for different Bi $6 s / \mathrm{O} p$ hybridization energies $\left(t_{s p \sigma}\right)$, for $t_{p p \pi}=-0.04 \mathrm{eV}, t_{p p \sigma}=0.63$ $\mathrm{eV}, U=3 \mathrm{eV}, Q=3.6 \mathrm{eV}$, and $E_{C F}=1 \mathrm{eV}$. Multiplets lose their intensities significantly with turning on the bismuthoxygen hybridization. The curves are shifted along the "Intensity" axis. . . . . . . . . . . . . . . . 102

A.1 The constructive Bragg reflections for monoclinic $\mathrm{BaBiO}_{3}$ with lattice parameters given in table 2.1 for $17.68^{\circ} \leq 2 \theta \leq$ $63.29^{\circ}$ calculated via Vesta program. To be continued on the next two pages. . . . . . . . . . . . . . . . . . 118 


\section{Acknowledgements}

First and foremost, I would like to express my sincere gratitude to my supervisor Professor George A. Sawatzky for his continuous support, patience, motivation, and immense knowledge. I could not have imagined having a better mentor and advisor for my Ph.D. study. His strong work ethic, neverending passion for knowledge, and his genuine care for the people around him have provided me with the best example of a great professional and human being.

Besides my supervisor, I would like to thank the rest of my thesis committee: Prof. Dough Bonn, Prof. Mona Berciu, and Prof. Mark Van Raamsdonk for serving as my committee members and for their insightful comments, encouragement and the stimulating discussions. I would like to, especially, thank Prof. Dough Bonn for giving me the opportunity to join the crystal growth laboratory and for his help and guidance through out the crystal growth project. I also thank Dr. Shun Chi for his tremendous help throughout the same project.

My sincere thanks also go to Dr. Robert Green without whom this work would not have seen the light. I would like to thank him for his continuous support and mentorship throughout the synchrotron x-ray measurements and also the exact diagonalization calculations. I sincerely thank Dr. Kateryna Foyevtsova for supporting the band structure calculation aspect of this work. I also thank Dr. Oleksandr Foyevtsov, Dr. Fengmiao Li, Dr. Ronny Sutarto, Dr. Ilya Elfimov, and Mr. Arash Khazraei for their precious support at different stages of this work.

And last but not least, I gratefully thank the Stewart Blusson quantum Matter Institute and the University of British Columbia for providing me with a vast number of resources throughout my study. 


\section{Dedication}

Dedicated to my husband, Ehsan, for his continuous love and patience, and to my family and friends for their encouragement and support. 


\section{Chapter 1}

\section{Bismuthates}

$\mathrm{BaBiO}_{3}$ was first made and introduced in $1963[20]$. It is a diamagnetic insulating perovskite compound with a reddish brown colour. From the beginning, its physics attracted attention due to its insulating mechanism which was believed to be the charge disproportionation of $\mathrm{Bi}^{+4}$ into $\mathrm{Bi}^{+3}$ and $\mathrm{Bi}^{+5}$. This description has been challenged recently both experimentally and theoretically.

In 1975, it was discovered that the material becomes superconducting with the critical temperature of $13 \mathrm{~K}$ when doped with holes by partially substituting bismuth with lead [21]. Later in 1988, a higher $T_{c}$ of $34 \mathrm{~K}$ was discovered for another hole doped compound by partially substituting barium with potassium [22]. It is one of the highest critical temperatures discovered for a three-dimensional compound without a transition metal ion. Despite this remarkable $T_{c}$, there have been many relatively quiet years regarding the bismuthate perhaps due to the revolutionary discovery of cuprates' superconductivity at about the same time in 1986 [23]. In this chapter, we review some of the key properties of the material and the main theories around them.

\subsection{Electronic structure}

From the beginning, it was generally believed that $\mathrm{BaBiO}_{3}$ is a valanceskipping compound with the true structure of $\mathrm{Ba}_{2} \mathrm{Bi}^{+3} \mathrm{Bi}^{+5} \mathrm{O}_{6}$. The main reasoning is that the nominal $\mathrm{Bi}^{+4}$ is not an energetically stable oxidation state for the bismuth atom. The electron configuration of bismuth is [Xe $4 f^{14} 5 d^{10} 6 s^{2} 6 p^{3}$; therefore, it would either lose its three $p$ electrons or the three $p$ plus its two $6 s$ electrons "skipping" the +4 state.

This conclusion was later supported by two important observations. First, the material was found to be insulating with a direct and an indirect gap of about $2 \mathrm{eV}$ and $0.25 \mathrm{eV}$, respectively [21, 24], and then in 1976 neutron diffraction found two distinct Bi-O bond lengths of $2.28 \AA$ and 2.12 $\AA[2,25]$. $\mathrm{BaBi}^{+4} \mathrm{O}_{3}$ has to be metallic due to the half-filled $6 s$ band. The above "charge-disproportionation" of bismuth cation into the +3 and +5 
states was able to explain the insulating behaviour and also the two different $\mathrm{Bi}-\mathrm{O}$ bond lengths.

However, the above charge-disproportionation picture has been questioned recently by several experiments as well as band structure calculations. Band structure calculations do not find a significant charge transfer between the two bismuth sites $4,26,27]$. X-ray photoemission measurements also don't give conclusive evidence for two different bismuth oxidation states [28-31]. Doubling the unit cell by the structural "bond-disproportionation" has been proposed as an alternative insulating mechanism by opening up a gap similar to the Peierls distortion mechanism but for a three-dimensional structure [4, 32, 33. In this picture, every other octahedron expands and collapses in a rock-salt pattern distortion.

The band structure calculation using density functional theory (DFT) shows how the strong hybridization between the deep $\mathrm{Bi} 6 s$ and the $\mathrm{O} 2 p$ bands pushes the $\mathrm{O} 2 p$ anti-bonding orbitals with $A_{1 g}$ symmetry above the Fermi energy [4]. Then due to the above bond- disproportionation distortion, pairs of oxygen holes form $A_{1 g}$ molecular orbitals around the Bi sites in the collapsed or short-bonded octahedra. Fig. 1.1 presents the density of states of $\mathrm{SrBiO}_{3}$ projected onto the $\mathrm{Bi}-6 s$ orbital and $A_{1 g}$ combinations of the O- $p_{\sigma}$ orbitals for the compressed and expanded $\mathrm{BiO}_{6}$ octahedra. The image has been reproduced by Ref. [5] from DFT calculation of Ref. [4]. As shown in Fig. 1.2 band structure calculation finds the electronic structure of $\mathrm{SrBiO}_{3}$ to be very similar to $\mathrm{BaBiO}_{3}$. The main difference is the larger gap in the $\mathrm{SrBiO}_{3}$ due to a larger structural distortion [4].

Fig. 1.1 shows that states of mainly O- $2 p$ character with $A_{1 g}$ symmetry generate a strong conduction band in the compressed octahedron. This band is much less prominent for the expanded octahedron. This demonstrates the bond-disproportion action which is due to the role of the oxygen $2 p$ holes and not the typically believed charge disproportionation of the bismuths.

In this picture, bismuths are all +3 and the other remaining hole resides on the three oxygen anions. This is a very different description electronically and hasn't yet been accepted unanimously by the community [7]. One objection is that the evidence for holes on the oxygens should clearly appear in experiments like $\mathrm{O} 1 s$ x-ray photoemission; however, the existing results have not been conclusive $[27,28,30,31,34$. In this work, we try to address this controversy by studying the electronic structure of oxygen anions experimentally with x-ray spectroscopy measurements and theoretically using exact diagonalization cluster calculations. 


\subsection{Electronic structure}
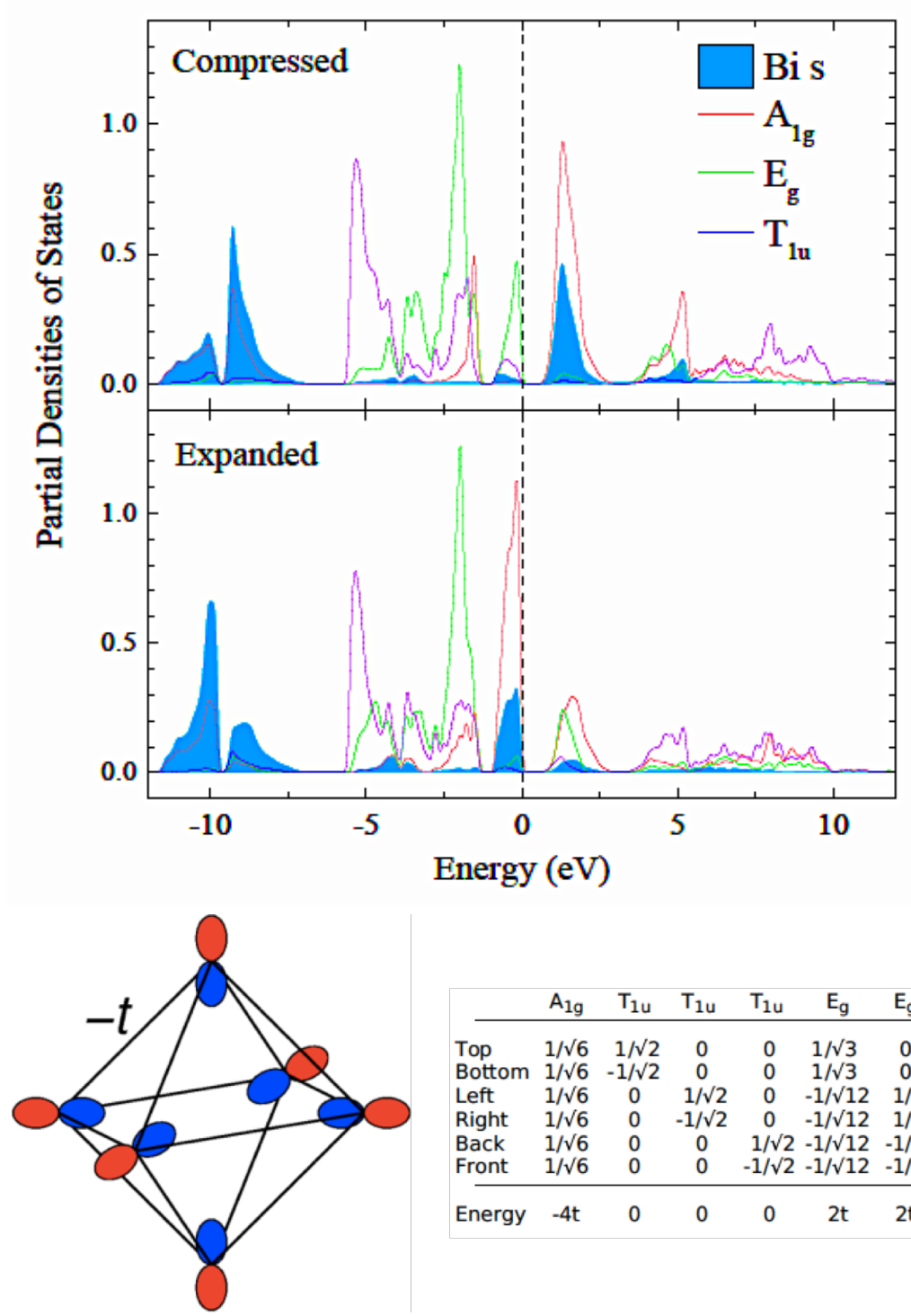

\begin{tabular}{lcccccc} 
& $\mathrm{A}_{1 \mathrm{~g}}$ & $\mathrm{~T}_{1 \mathrm{u}}$ & $\mathrm{T}_{1 \mathrm{u}}$ & $\mathrm{T}_{1 \mathrm{u}}$ & $\mathrm{E}_{\mathrm{g}}$ & $\mathrm{E}_{\mathrm{g}}$ \\
\hline Top & $1 / \sqrt{ } 6$ & $1 / \sqrt{ } 2$ & 0 & 0 & $1 / \sqrt{ } 3$ & 0 \\
Bottom & $1 / \sqrt{ } 6$ & $-1 / \sqrt{ } 2$ & 0 & 0 & $1 / \sqrt{ } 3$ & 0 \\
Left & $1 / \sqrt{ } 6$ & 0 & $1 / \sqrt{ } 2$ & 0 & $-1 / \sqrt{ } 12$ & $1 / 2$ \\
Right & $1 / \sqrt{ } 6$ & 0 & $-1 / \sqrt{ } 2$ & 0 & $-1 / \sqrt{ } 12$ & $1 / 2$ \\
Back & $1 / \sqrt{ } 6$ & 0 & 0 & $1 / \sqrt{ } 2$ & $-1 / \sqrt{ } 12$ & $-1 / 2$ \\
Front & $1 / \sqrt{ } 6$ & 0 & 0 & $-1 / \sqrt{ } 2$ & $-1 / \sqrt{ } 12$ & $-1 / 2$ \\
\hline Energy & $-4 \mathrm{t}$ & 0 & 0 & 0 & $2 \mathrm{t}$ & $2 \mathrm{t}$
\end{tabular}

Figure 1.1: Top: Density of states of a compressed and expanded $\mathrm{BiO}_{6}$ octahedron projected on to the $\mathrm{Bi}-6 s$ orbital and combinations of the $\mathrm{O}$ $p \sigma$ orbitals for $\mathrm{SrBiO}_{3}$ structure from Ref. [4]'s DFT(LDA) calculations [4, 5]. Bottom: The octahedron with O- $p \sigma$ orbitals at the corners hybridized through nearest-neighbor hopping integrals of $-t$ and the corresponding eigenstates in the table for different symmetries [4] . 


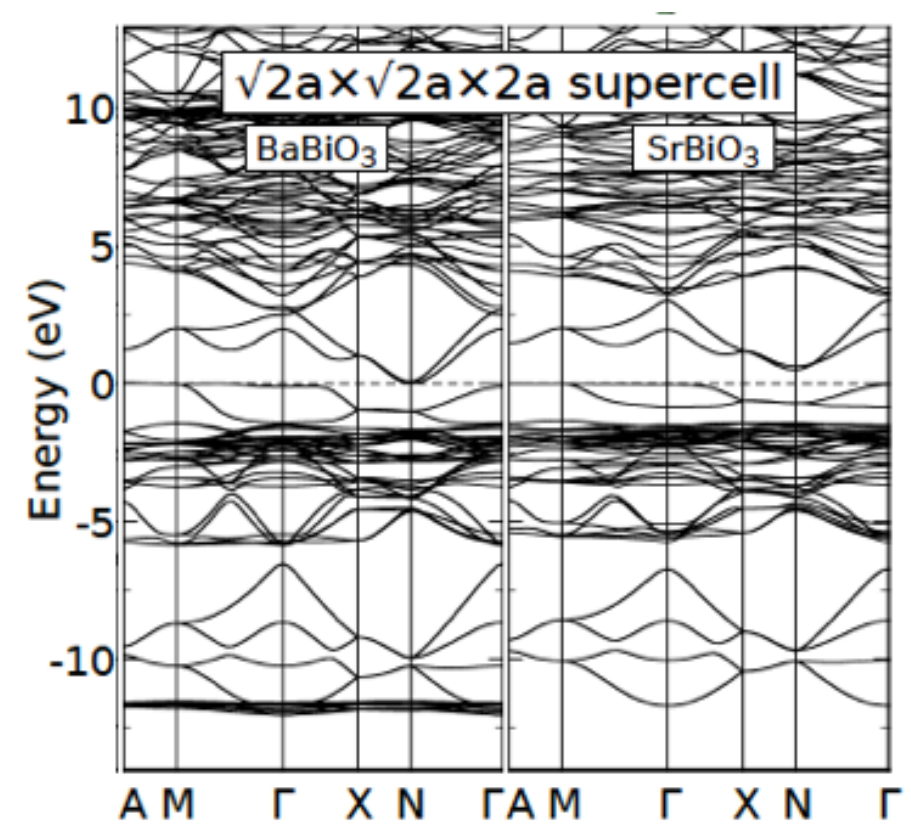

Figure 1.2: DFT(LDA) band structures of $\mathrm{BaBiO}_{3}$ and $\mathrm{SrBiO}_{3}$ calculated with a supercell of dimensions $\sqrt{2} a \times \sqrt{2} a \times \sqrt{2} a$. The band structures are almost identical around the Fermi energy except for a larger gap in $\mathrm{SrBiO}_{3}$ due to its more prominent structural distortions. The Fermi energy is set to zero [4]. 
(a)

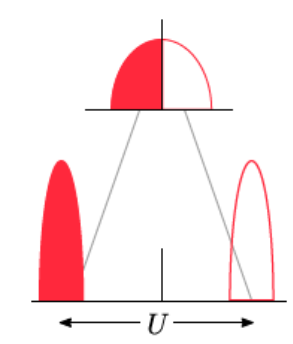

(b)

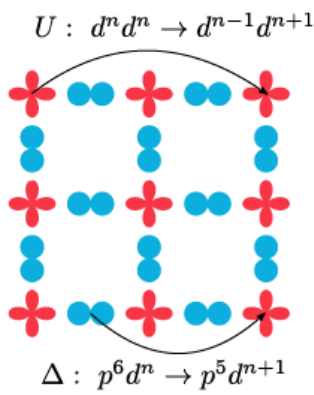

(c)

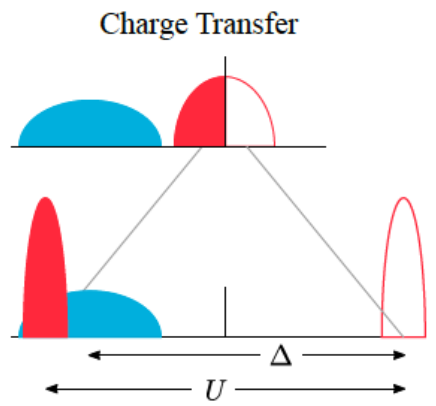

Figure 1.3: a: Mott-Hubbard insulators where the gap is determined by the Coulomb energy $U$ versus c: charge-transfer insulators where the gap is determined by $\Delta$ which is smaller than $U$. b: charge fluctuations corresponding to the energies $\Delta$ and $U$. The blue and red orbitals represent the ligand oxygen $p$, and the transition metal $d$ orbitals, respectively $[5,6$.

\subsubsection{Bismuthates and Nickelates}

The above breathing type lattice distortion is similar to the one in the nickelate perovskite where every other octahedron expands and compresses alternatively. Nickelate perovskite, $R \mathrm{NiO}_{3}, R=$ Rare earth, is known as a charge transfer insulator [35]. In correlated transition metal compounds, the charge transfer energy, $\Delta$, is defined as the energy required to move an electron from a ligand oxygen $2 p$ orbital to the cation $3 d$ orbital or, equivalently, the energy cost for the transition $p^{6} d^{n} \rightarrow p^{5} d^{n+1}$.

As opposed to the Mott-Hubbard insulators, charge transfer insulators have a smaller charge transfer energy than the Coulomb energy $U_{d d}$ and therefore the gap is determined by $\Delta$, not $U$. The distinction between these two types of insulators is schematically presented in Fig. 1.3. This was first introduced in the Zaanen-Sawatzky-Allen (ZSA) theory in 1985 [6]. In recent years, it has been shown that nickelates are in fact negative charge transfer insulators meaning that the $p^{5} d^{n+1}$ state has a lower energy than the $p^{6} d^{n}$ state, so the ground state is better described as $\underline{L} d^{n+1}$ where $\underline{L}$ denotes a hole on the ligands [6, 35, 36].

Even though the above description is focused on strongly correlated transition metal compounds, the non-strongly-correlated bismuthates show similar behaviours to the negative charge transfer compounds like nickelates. As mentioned above, both materials exhibit similar breathing type distortion 

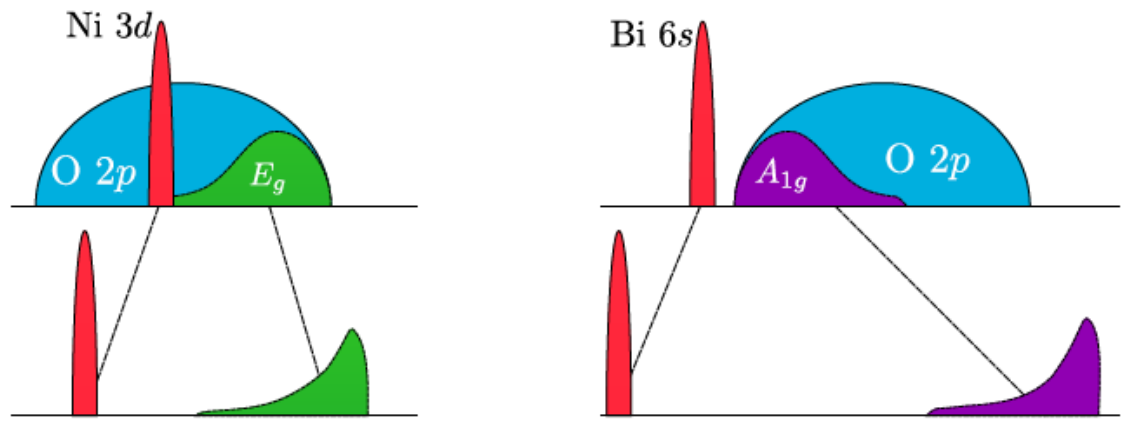

Figure 1.4: The schematic illustration of the perovskite nickelate (on the left) and bismuthate (on the right) electronic structures. In the bismuthate, the deep Bi- $6 s$ states with the $A_{1 g}$ symmetry hybridize strongly with the combination of the $\mathrm{O}_{2} p$ states with the same symmetry. Consequently, the state of $A_{1 g}$ symmetry and mainly $\mathrm{O}_{2} p$ character are pushed above the Fermi level. In the nickelate, the situation is similar but the cation states are the $\mathrm{Ni}-3 d$ states with the $E_{g}$ symmetry which again mix with the $\mathrm{O}_{2} p$ states with the same symmetry [5].

with alternating expanded and compressed octahedra responsible for their insulating phase.

In nickelates, the hybridization situation is also very similar to bismuthates except that in nickelates the $\mathrm{Ni}-d$ states are playing the role, so it is the $E_{g}$ symmetry of the molecular orbitals involved rather than the $A_{1 g}$ symmetry states. In other words, in nickelates, the $E_{g}$ symmetry Ni-d bands hybridize strongly with the $E_{g}$ symmetry oxygen $p$ states which results in the anti-bonding oxygen hole states with the $E_{g}$ symmetry. They then form molecular orbitals with the same symmetry around the short bonded $\mathrm{Ni}$ cations in the compressed octahedron [5] similar to what happens in the bismuthates. The schematic similarities of the electronic structure of these two perovskites are shown in Fig. 1.4. The fact that these two perovskites exhibit striking similarities even though they have very different cations demonstrates the importance of the role of oxygen in describing the electronic properties of perovskites. 


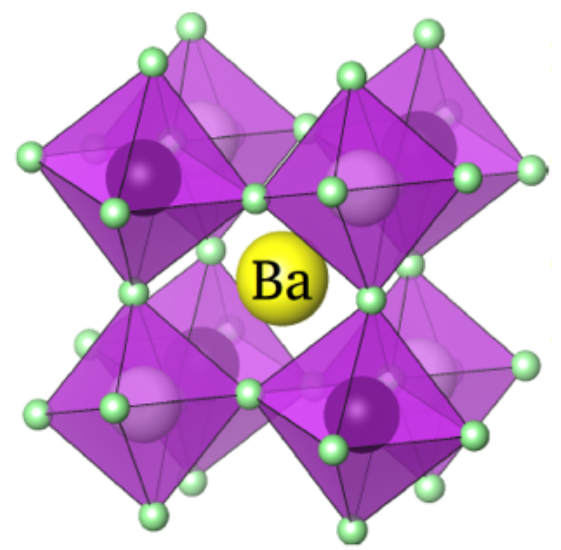

Figure 1.5: The crystal structure of perovskite $\mathrm{BaBiO}_{3}$. The green balls at the corners of the octahedra represent the oxygen anions. The black and white balls at the centers of the octahedra represent the bismuth cations in expanded and collapsed octahedra, respectively [4].

\subsection{Crystal Structure}

The structure of the perovskite $\mathrm{BaBiO}_{3}$ has been determined using x-ray and neutron powder diffraction techniques over temperatures from $4.2 \mathrm{~K}$ to $973 \mathrm{~K}[7,37$. where it goes through three main structural transitions. The material is monoclinic at temperatures from $4.2 \mathrm{~K}$ to $430 \mathrm{~K}$; however, the space group changes from $P 2 / m$ to $I 2 / m$ at about $140 \mathrm{~K}$. Then at about $430 \mathrm{~K}$, it transitions to a rhombohedral structure in the $R 3$ space group up until $820 \mathrm{~K}$ where it finally becomes cubic in the $P m 3 m$ space group. As mentioned before, the neutron diffraction also finds two different Bi-O bond lengths of $2.28 \AA$ and $2.12 \AA$ or two distinct, expanded and collapsed, octahedra in the material at room temperature 2,37 . The structure at room temperature is illustrated in Fig. 1.5.

For perovskite compounds, $\mathrm{ABO}_{3}$, the Goldschmidt tolerance factor $t$ is defined as:

$$
t=\frac{r_{A}+r_{0}}{\sqrt{2}\left(r_{B}+r_{0}\right)}
$$

where $r_{A}, r_{B}$, and $r_{O}$ are the radii of the cation $A$, cation $B$ and the oxygen anion, respectively. The deviation from 1 of $t$ signifies the amount of 
distortion in the lattice from an ideal cubic structure. The further apart the tolerance factor from 1 , the more distorted the structure is. The tolerance factor for $\mathrm{BaBiO}_{3}$ is less than 1 at about 0.9 which results in tilted octahedra and a distorted perovskite structure [38, 39].

\subsection{Superconductivity}

The fact that $\mathrm{BaBiO}_{3}$ is a diamagnetic insulator motivated people to look for an insulator-to-metal transition in $\mathrm{BaBi}_{x} \mathrm{~Pb}_{1-x} \mathrm{O}_{3}$ compounds since $\mathrm{BaPbO}_{3}$ was a well-known metal. This search, more than a decade later, in 1975, led to the discovery of superconductivity in the hole doped $\mathrm{BaBi}_{x} \mathrm{~Pb}_{1-x} \mathrm{O}_{3}$, for $x=0.05-0.3$, with the highest transition temperature of $T_{c}=13 \mathrm{~K}$ for $\mathrm{x}=0.3[21]$. It was the highest observed $T_{c}$ for a material with no transition metal until 1988 when interestingly the record broke by the same family of material, $\mathrm{Ba}_{x} \mathrm{~K}_{1-x} \mathrm{BiO}_{3}$, with the highest $T_{c}$ of $34 \mathrm{~K}$ for $\mathrm{x}=0.35$ [22]. This was a remarkable discovery mainly because it was the highest observed $T_{c}$ for a three-dimensional material with no transition metal and with a minimal electronic density of states around the Fermi level [40].

The phase diagrams of $\mathrm{Ba}_{x} \mathrm{~K}_{1-x} \mathrm{BiO}_{3}$ and $\mathrm{BaBi}_{x} \mathrm{~Pb}_{1-x} \mathrm{O}_{3}$ compounds are shown in Fig. 1.7 [7]. The lead doped compound generally has more complex and less understood phase relationships mainly due to the lack of phase purity at different $x$ values. In general, the tetragonal phase is identified as the superconducting phase for $x=0.05-0.3$ [37]. However, mixtures of tetragonal and orthorhombic structures are almost always detected for those values of $x[37,41]$. A recent ultrafast experiment also reports finding evidence for the coexistence of CDW order and superconductivity for the underdoped $\mathrm{BaBi}_{x} \mathrm{~Pb}_{1-x} \mathrm{O}_{3}$ composition 42 . This is especially notable since CDW correlations have also been recently observed for some underdoped cuprate materials [43, 44]. This gives more evidence for the possible commonalities between the physics of these two superconducting families.

Understanding the phase relationships of the potassium substituted compound, $\mathrm{Ba}_{x} \mathrm{~K}_{1-x} \mathrm{BiO}_{3}$, is less complicated thanks to the availability of the more high-quality single phase crystals. When the potassium is substituted for barium, the tolerance factor $t$ increases from $t=0.9$ for the pure compound because $\mathrm{K}^{1+}$ is larger than the $\mathrm{Ba}^{2+}$. Thus, the doped material gets less distorted and ultimately becomes cubic at room temperature. However, it was later observed that the structure at the superconducting temperatures is, in fact, tetragonal $(I 4 / \mathrm{mcm})$ with tilted octahedra [45]. The summary of $\mathrm{Ba}_{x} \mathrm{~K}_{1-x} \mathrm{BiO}_{3}$ properties is presented in table 1.6 . 


\begin{tabular}{|c|c|}
\hline Density of states $\left[N^{*}(0)\right]$ & $0.45-0.67$ \\
\hline $\begin{array}{l}\text { Symmetry of } \\
\text { superconducting state }\end{array}$ & s Wave \\
\hline Dimensionality & $3 \mathrm{D}$ \\
\hline Range of $T_{c}$ & $5-34 \mathrm{~K}$ \\
\hline Pressure dependence of $T_{c}$ & $-(9 \pm 02) \times 10^{-5} \mathrm{Kbar}^{-1}$ \\
\hline Specific heat jump at $T_{c}$ & $4-5 \mathrm{~mJ} \mathrm{~mol}^{-1} \mathrm{~K}^{-2}$ \\
\hline Energy gap & $2 \Delta(0)=4.0 \pm 0.1 \mathrm{kT}_{\mathrm{c}}(6.2 \mathrm{meV})$ \\
\hline Coherence length & $\sim 50 \AA$ \\
\hline London penetration depth & $5500 \pm 100 \AA$ \\
\hline Debye temperature & $\sim 210 \mathrm{~K}$ \\
\hline Resistivity & $2-45 \mathrm{~m}-\Omega-\mathrm{m}$ at $298 \mathrm{~K}$ \\
\hline Magnetic susceptibility & $\chi_{\text {Pauli }}=2.3 \times 10^{-5} \mathrm{emu} / \mathrm{mole}$ \\
\hline $\begin{array}{l}\text { Carrier concentration from } \\
\text { Hall data }\end{array}$ & $\begin{array}{l}\sim 10^{-21} / \mathrm{cm}^{3} \text { or } 0.3-0.6 / \text { unit cell } \\
\text { ( } \mathrm{T} \text { independent) }\end{array}$ \\
\hline Isotope-effect exponent. & 0.48 \\
\hline
\end{tabular}

Figure 1.6: Summary of $\mathrm{Ba}_{x} \mathrm{~K}_{1-x} \mathrm{BiO}_{3}$ properties taken from Ref. [7].

There have been some other superconducting compositions discovered in this family such as $\mathrm{Sr}_{x} \mathrm{~K}_{1-x} \mathrm{BiO}_{3}$ and $\mathrm{Ba}_{x} \mathrm{Rb}_{1-x} \mathrm{BiO}_{3}$ with the highest $T_{c}$ 's of $12 \mathrm{~K}$ and $29 \mathrm{~K}$ [46, 47], respectively. These are all $s$-wave superconductors and their superconductivity mechanism is generally attributed to the bipolaronic situation implicit in the said CDW or the charge/bond disproportionation picture 48 51].

\subsubsection{Bismuthates and Cuprates}

Bismuthate perovskites have some notable similarities with some other interesting electronic materials such as nickelates, which was discussed in the previous section, and also with the high- $T_{c}$ cuprate superconductors. Both these high- $T_{c}$ superconductors are described by strongly hybridized bands of the ligand $\mathrm{O} 2 p$ and the cation valence states about the Fermi level $(\mathrm{Cu}$ $3 d$ for the cuprates and $\mathrm{Bi} / \mathrm{Pb} 6 s$ for the bismuthates).

However, in cuprates the hybridization is mainly between the $\mathrm{Cu} 3 d\left(x^{2}-\right.$ $\left.y^{2}\right)$ and the oxygen $2 p$ states formed from the four ligand oxygen atoms, while in the bismuthate it is between the spherically symmetric $\mathrm{Bi} / \mathrm{Pb} 6 s$ states 


\subsection{Superconductivity}
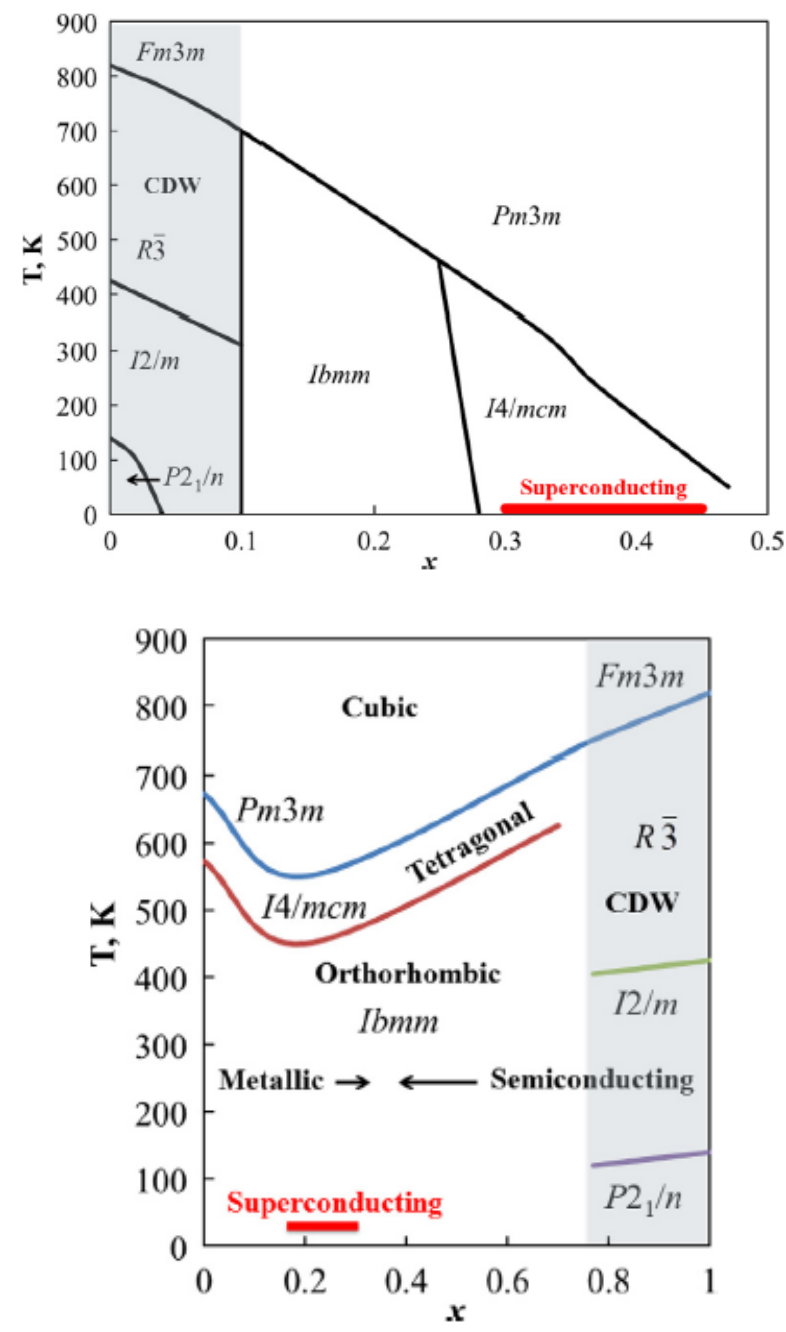

Figure 1.7: The phase diagrams of top: $\mathrm{Ba}_{1-x} \mathrm{~K}_{x} \mathrm{BiO}_{3}$ and bottom: $\mathrm{BaBi}_{1-x} \mathrm{~Pb}_{x} \mathrm{O}_{3}$ compounds. Due to the lack of phase purity for $\mathrm{BaBi}_{1-x} \mathrm{~Pb}_{x} \mathrm{O}_{3}$ samples and hence the non-conclusive results the $\mathrm{BaBi}_{1-x} \mathrm{~Pb}_{x} \mathrm{O}_{3}$ phase diagram is perhaps only qualitatively correct [7]. 
and the $2 p$ orbitals of the six ligand oxygens $[52,53$. In both cases, this strongly hybridized band splits and opens up a gap giving rise to an insulating state. However, while in the diamagnetic bismuthates it is due to the above CDW or bipolaronic type of ordering, in cuprates, the large on-site $d-d$ Coulomb repulsion causes the un-doped material with configuration $d^{9}(s=1 / 2)$ and a full $\mathrm{O} 2 p$ band (i.e. a positive charge transfer gap) to form local moments which at low temperatures form a stable antiferromagnetic ordered structure and a conductivity gap is determined by the positive charge transfer gap. Upon hole doping however the holes go into a linear combination of $\mathrm{O} 2 p$ orbitals of $d_{x^{2}-y^{2}}$ symmetry strongly hybridizing with the $\mathrm{Cu} 3 d$ orbital of the same symmetry and together these two holes (one on $\mathrm{Cu}$ and One on $\mathrm{O} 2 p$ ) form a singlet and form a metallic state.

The hole-doped cuprates and bismuthates both have a low density of states around the Fermi level compared to their superconducting critical temperatures [52]. Perhaps that is why the bismuthate superconductors have also been often categorized as "unconventional" or "possibly unconventional" superconductors [7]. However, a more recent modified calculation claims that they find a high $T_{c}$ consistent with their "correlation-enhanced electron-phonon coupling" model[51]. 


\section{Chapter 2}

\section{$\mathrm{BaBiO}_{3}$ Single Crystal Growth}

In this chapter, we discuss how we developed a routine to grow single crystals of $\mathrm{BaBiO}_{3}$ with sizes up to $3 \times 3 \times 1 \mathrm{~mm}^{3}$. We also present the results of the characterization techniques used to measure their chemical composition and their structural and transport properties.

\section{$2.1 \quad$ Introduction}

A single crystal or monocrystalline solid is typically defined as a material that maintains translational symmetry over macroscopic distances of bigger than $100 \mu \mathrm{m}$. It requires an absence of defects associated with grain boundaries. Because entropic effects favour the presence of such imperfections, perfect single crystals of reasonable macroscopic sizes are rare in nature, and are also challenging to make in the laboratory [54] [55].

Single crystals are preferred and sometimes necessary for structural determination or intrinsic property studies. For instance, in conductivity measurements, insulating grain boundaries can give rise to semiconducting properties for metallic oxides resulting in inconclusive outcomes. Even in our specific case of bismuthates, initially, the resistivity of the $\mathrm{Ba}_{x} \mathrm{~K}_{1-x} \mathrm{BiO}_{3}$ polycrystalline samples above $T_{c}$ found to decrease with increasing temperature. However later, resistivity measurements on the single crystal samples, showed the resistivity indeed increases with increasing the temperature as expected for a metal[7][56].

Many crystal growth techniques have been developed over the years. Each of these techniques has its advantages and disadvantages and is used based on the properties of the material and the application requirements. These techniques can be categorized into the four following main methods [8]:

- Solid State Growth: From a mixture of solids with no melting involved such as Strain Anneal and Sintering techniques. These techniques usu- 


\subsection{Introduction}

ally result in polycrystalline materials with at best tiny single crystal regions.

- Vapour Phase Growth: From solid to gas (vapour) to crystal. These deposition techniques can be made on a lattice matched single crystal substrate resulting in epitaxial thin film growth such as Pulsed Laser Deposition (PLD) and Molecular Beam Epitaxy (MBE) techniques. This method can also be used for growing bulk single crystals.

- Melt Growth: From solid to liquid to crystal. In these techniques, the polycrystalline material is heated in a non-reacting crucible to above its melting point and then is slowly cooled down to form crystals. Various additional steps can be taken to enhance the crystal formation and quality such as inserting a seed crystal into the melt and pulling and rotating the seed to form a crystallization centre, or employing a temperature gradient along the crucible which are known as the Czochralski and Bridgman techniques, respectively.

- Solution Growth: In these techniques, a saturated solution is prepared by dissolving the components of the desired crystal in a solvent at a temperature above the saturation temperature. Then the supersaturated solution is cooled down for the crystal to precipitate. Crystallization can happen by spontaneous nucleation or can be enhanced by using a seed.

In growing the bismuthate single crystal, the electrochemical method (solution growth) has often been used and proven successful in growing the superconducting $\mathrm{Ba}_{x} \mathrm{~K}_{1-x} \mathrm{BiO}_{3}$ crystals as big as $5 \times 4 \times 3 \mathrm{~mm}^{3}$ [57][58]. In this technique typically a mixture of $\mathrm{Ba}(\mathrm{OH})_{2} \cdot 8 \mathrm{H}_{2} \mathrm{O}$ and $\mathrm{Bi}_{2} \mathrm{O}_{3}$ powders is used in molten $\mathrm{KOH}$ where the weight ratio of barium hydroxide and the potassium hydroxide determines the amount of potassium substitution. However, due to the involved potassium, this technique is not suitable for growing pure $\mathrm{BaBiO}_{3}$ single crystal which is the material of our main interest in this study as the parent compound.

There are not as many studies on growing the parent $\mathrm{BaBiO}_{3}$ single crystals compared to its doped superconducting compounds. However, floating zone [59] and molten flux techniques [9] have been reported to be successful in growing such single crystals. The floating zone method requires specific equipment which was not available in our laboratory at the time. Moreover, the referred report [59] lacks any stoichiometry or detailed structural analysis to judge the quality of the resultant crystals. Therefore, we took the 


\subsection{Introduction}

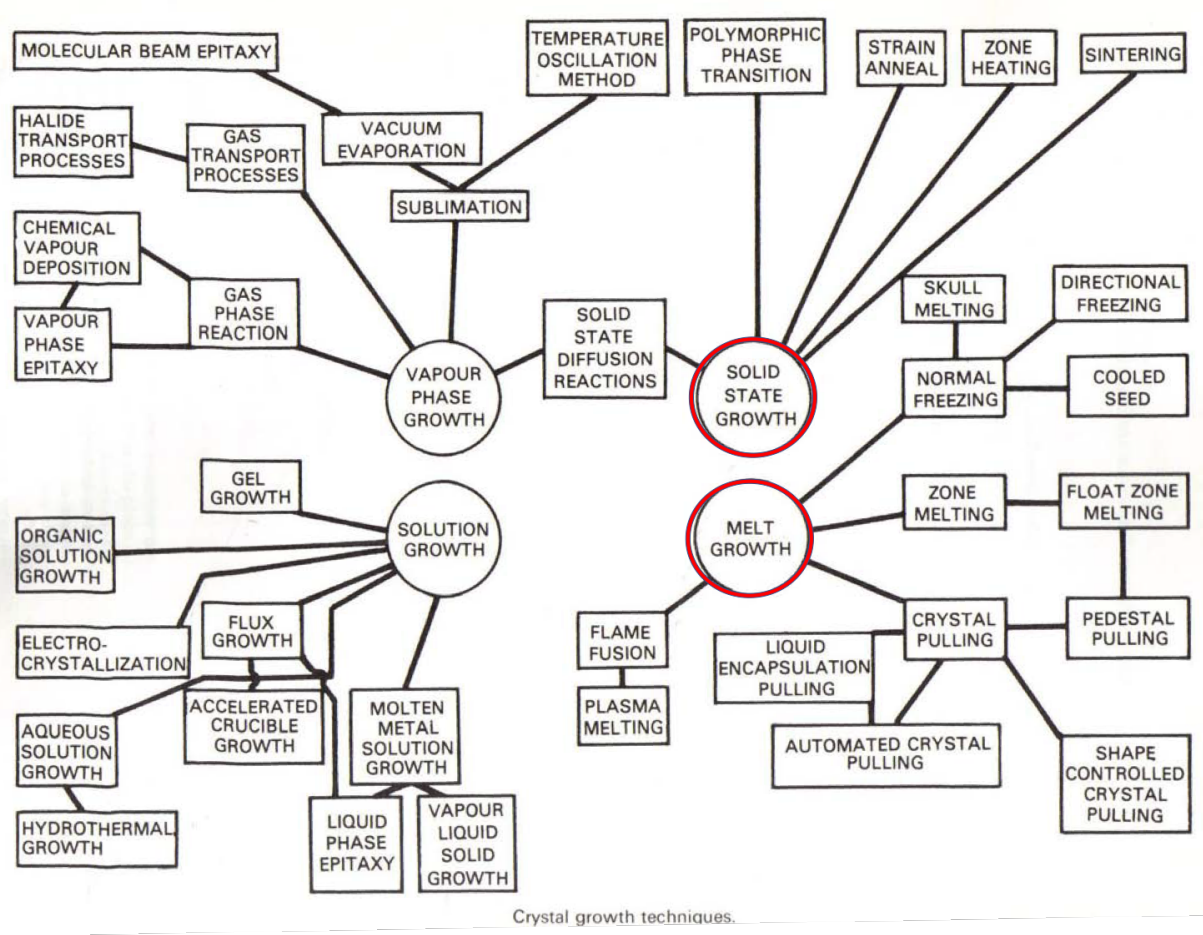

Figure 2.1: An overview of the most used crystal-growth techniques [8]. These techniques fall into four main categories: solid-state growth, vapour phase growth, melt growth and solution growth. In this work a combination of solid-state+melt growth was ultimately used to grow $\mathrm{BaBiO}_{3}$ crystals.

other approach of slow-cooling the molten mixture by I. Shiozaki and H. Ishii [9] as a guidance to start. As shown below, we realized this recipe does not give us our desired big high-quality single crystals either. Therefore we made some modifications to be able to achieve our desired result.

Growth from the melt by solidification is one of the oldest and also most used single crystal techniques for growing large single crystals. The first systematic study of such crystal growth was carried out by Tamman in 1899 . He tracked the rate of the crystal solidification of an augite composition from its melt. He found with decreasing the temperature of the melt, the crystallization rate increases to a maximum and then decreases. A more rigorous theory was developed later by Volmer and Marde in 1931 [60]. The melting technique is most useful and straightforward for materials that melt 
congruently.

Congruent melting, which is contrasted with the incongruent melting, is the thermal behaviour of intermediate compounds in a temperaturecomposition phase diagram. An intermediate compound is a combination of the end members of the phase diagram between the two solids. Congruent melting is when one solid phase transforms to another solid phase and a liquid phase both of the same chemical compositions as of the original composition.

Two main factors control the size and purity of the resultant single crystal: nucleation rate and growth rate. If nucleation is rapid compared to the overall growth, small single crystals or polycrystalline samples will result. On the other hand, if the nucleation is slow and the growth is fast large single crystals will form. However, note that defect concentration tends to increase with increasing the growth rate. Therefore, in order to get high-quality crystals with a reasonable size, both the growth rate and the nucleation rate should remain small.

There are several techniques within the melt-growth category to lower the nucleation rate by limiting the number of nucleation sites: slow cooling the melt, introducing a temperature gradient, introducing a seed crystal, and moving the crystal-melt interface [61]. In this work, we start by solidstate sintering in order to synthesize the starting polycrystalline $\mathrm{BaBiO}_{3}$, and then the single crystal is grown by slow cooling the congruent-melt. The development of this procedure is explained below.

\subsection{Single Crystal Growth Procedure}

\section{First Attempt}

$\mathrm{BaCO}_{3}(99.9 \%)$ and $\mathrm{Bi}_{2} \mathrm{O}_{3}(99.9 \%)$ powders were mixed in the stoichiometric molar ratio of $2: 1$ based on the Eq. $2.1^{1}$

The mixture was then ground thoroughly until a fine homogenous mixture was achieved.

According to Material Safety Data Sheet (MSDS), bismuth trioxide is "slightly hazardous in case of eye contact (irritant), of ingestion, of inhalation" and barium carbonate is "hazardous in case of skin contact (irritant),

\footnotetext{
${ }^{1}$ It has been suggested that adding a $10 \%$ excess barium to the starting mixture helps avoid self-strain in the crystals 9 . For the first three attempts, we used the two mixtures (the stoichiometric, and the Ba-rich) at two separate crucibles but with the other exact same conditions, we did not observe any difference, so we proceeded with the stoichiometric mixture.
} 
of eye contact (irritant), of ingestion, of inhalation". Therefore, proper safety measures have to be taken in handling and storing these chemical materials. The grinding must be performed in a cool well-ventilated area.

$$
2 \mathrm{BaCO}_{3}+\mathrm{Bi}_{2} \mathrm{O}_{3}+\frac{1}{2} \mathrm{O}_{2} \rightarrow 2 \mathrm{BaBiO}_{3}+2 \mathrm{CO}_{2}
$$

The resulting powder was calcined in still air in a furnace at $850^{\circ} \mathrm{C}$ for 5 hours in a $10 \mathrm{~mm}$ Outer Diameter (OD) alumina crucible. A home-made tube furnace with a Lindberg digital controller with the maximum available temperature of $1200^{\circ} \mathrm{C}$ was used. No temperature gradient over the length and width of the crucible was detected. Note, the furnace was calibrated using a K-type standard thermocouple before each attempt. All the temperatures mentioned here are the true temperatures after the calibration, not the read numbers.

The mixture was heated at the centre of the furnace to a temperature higher than the $\mathrm{BaBiO}_{3}$ melting point of $1040^{\circ} \mathrm{C}[62]$ and then was cooled down slowly with the rates given in Figure 2.2 following the reference 9 . As shown in Fig. 2.3, this attempt was not successful in growing $\mathrm{BaBiO}_{3}$ single crystals as the Laue diffraction result suggests.

In x-ray Laue diffraction technique, the sample is irradiated by an $\mathrm{x}$ ray beam of continuous wavelength ("white" x-ray) while held stationary. The crystal reflects beams for which the Bragg condition is satisfied and a constructive diffraction takes place. The diffraction pattern of a single crystal should be a series of spots with information about its orientation and symmetry. The Laue method is convenient for a rapid assessment of a grown crystal. 


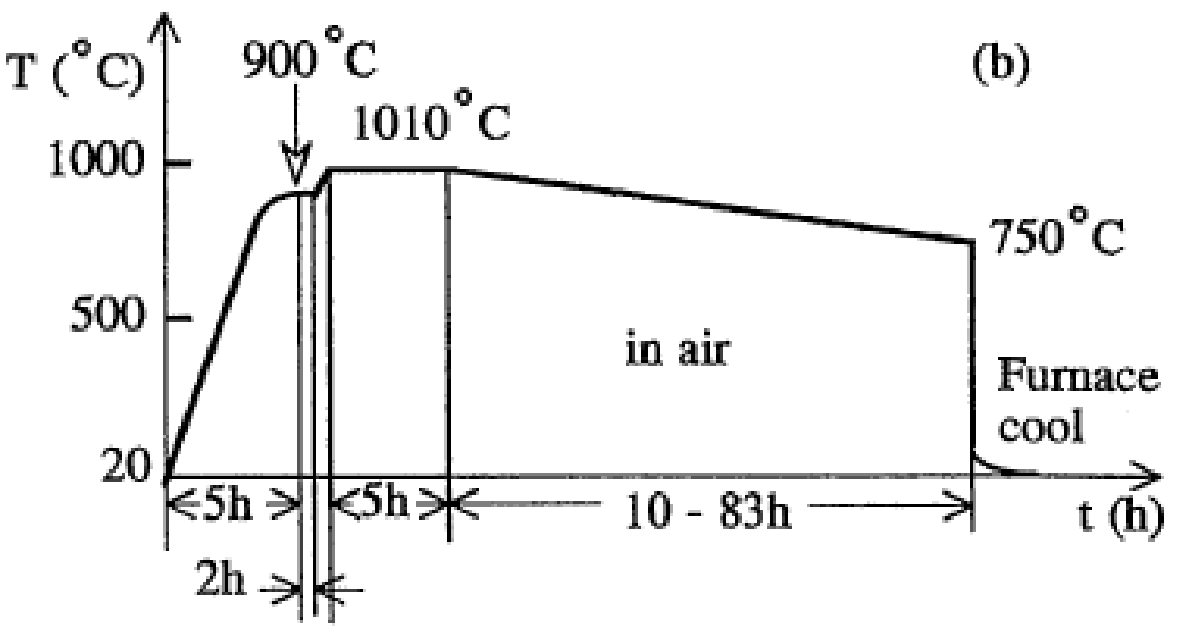

Figure 2.2: Temperature-steps for the $\mathrm{BaBiO}_{3}$ single crystal growth as given by Ref. [9]. (The highest temperature of $1010^{\circ} \mathrm{C}$ in the plot has to be an error. Based on the text and the $\mathrm{BaBiO}_{3}$ melting temperature of $1040^{\circ}$ $\mathrm{C}$, it has to be $1100^{\circ} \mathrm{C}$.) 

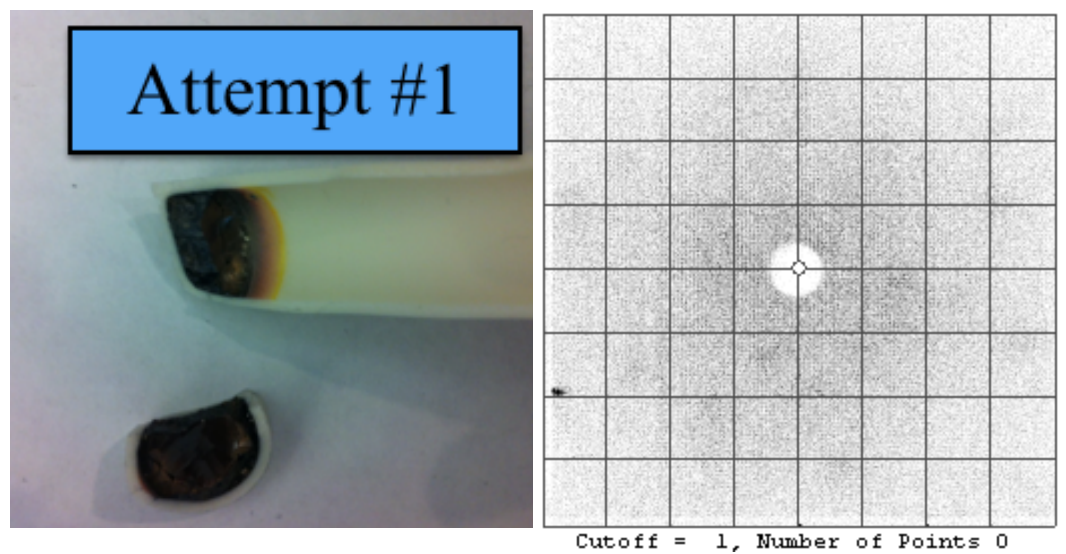

Figure 2.3: First growth attempt and its Laue-gram. The result was not single crystal. (unsuccessful)

\section{Second Attempt}

Trying to understand what the problem was in the last growth, we noticed in the reference paper [9] there was a temperature difference of about $3^{\circ} / \mathrm{cm}$ along the length of the crucible that was not included in our growth. Introducing a temperature gradient is, in general, a well-known part in many crystal growth techniques such as in Bridgman or Zone Melting methods. The idea is that when we start cooling down, the lower part of the melt with a lower temperature reaches the melting point sooner and therefore crystallizes earlier and then it can induce nucleation in the upper part of the melt.

Therefore, for the second attempt, we tried to apply such a gradient. In order to introduce this temperature gradient over the length of the crucible we designed and made a sloped insulating ceramic disk with a $7 \mathrm{~cm}$ outer thickness at the furnace wall and $1 \mathrm{~cm}$ inner thickness around the crucible. It is shown in Fig. 2.4. This way we achieved a temperature difference of about $9^{\circ} \mathrm{C}$ across the bottom two centimetres of the crucible. 

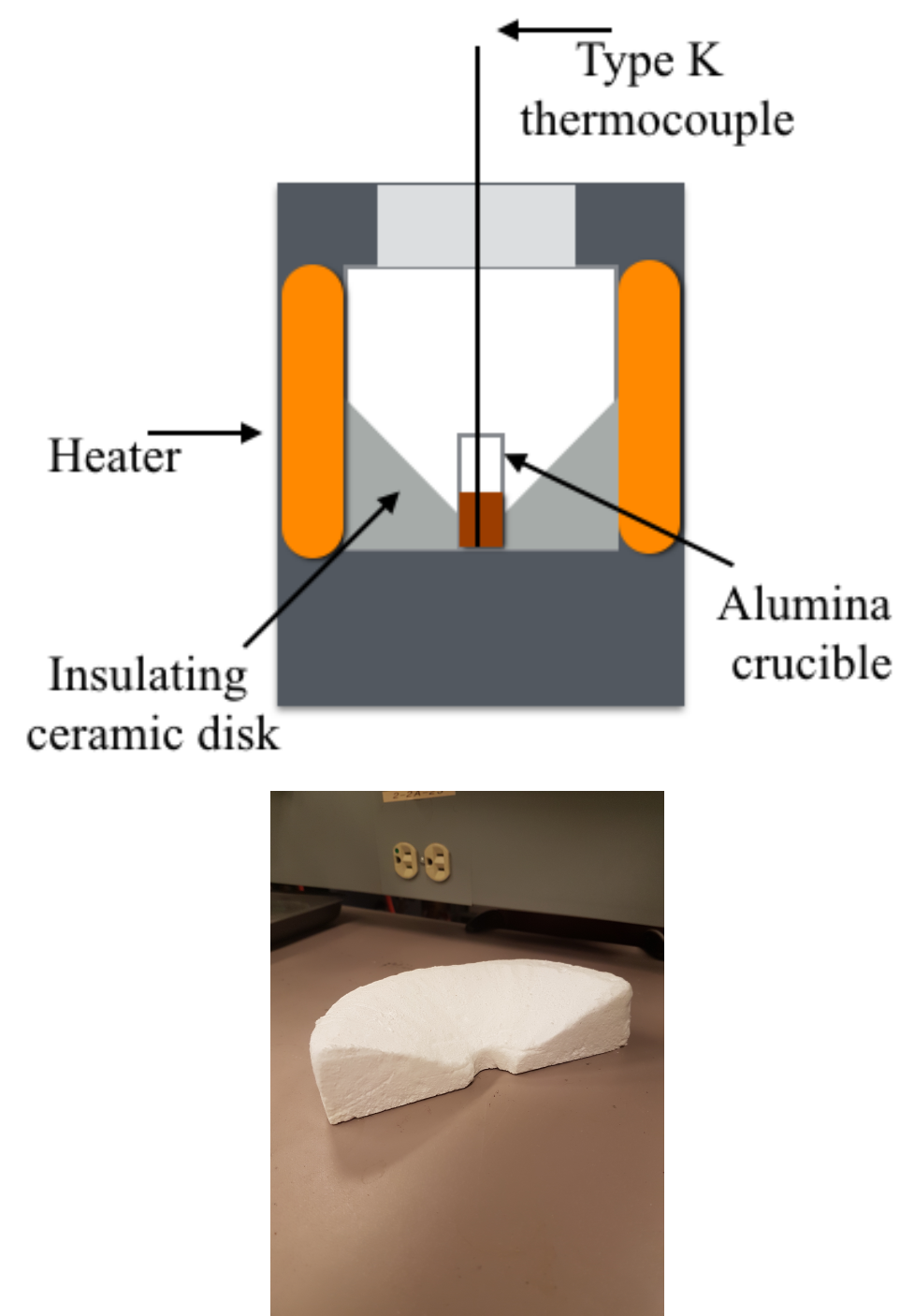

Figure 2.4: The construction of the furnace (top) with the insulating ceramic disk (bottom) to introduce a temperature gradient along the crucible for the second attempt.

The rest of the procedure was the same as the first try. As shown in Fig. 2.5, the growth was not successful either and the result was almost the same as the previous attempt. As realized later, that is because the crystal 
growth happens only at the top one to two millimetres of the melt due to the required air and oxygen exposure. Therefore, the temperature gradient does not help in this growth. The sloped disk was removed for the next attempts.

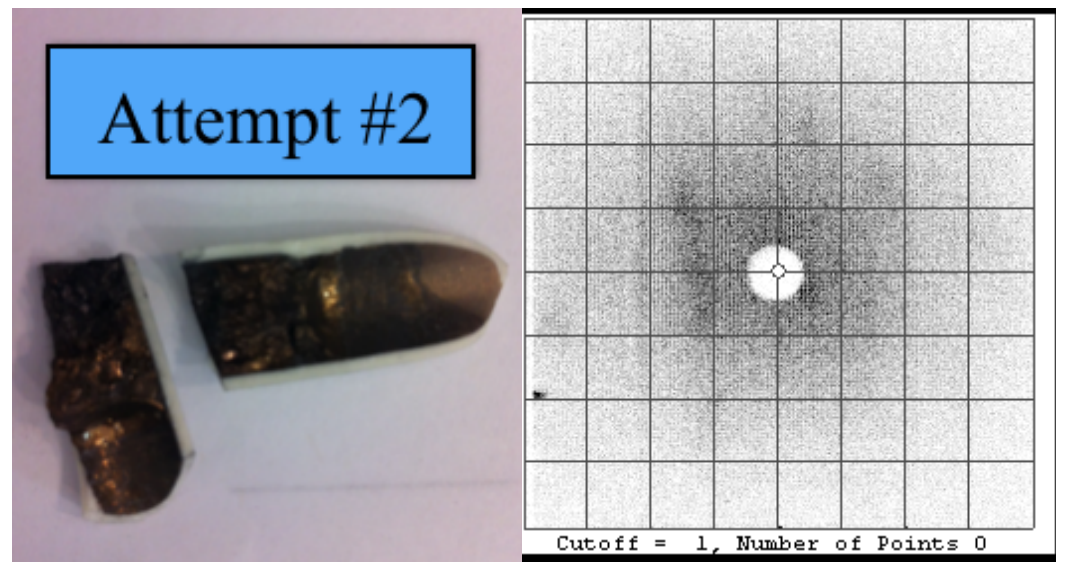

Figure 2.5: Second growth attempt and its Laue-gram. Here the previous procedure was repeated but a temperature gradient was also introduced along the crucible. The result was not single crystal. (unsuccessful)

\section{Third Attempt}

For this growth, we realized the calcined mixed powder does not look homogenous after being taken out of the furnace. It was dark grey with some shimmery brown patches. In order to start with a more homogeneous material we broke and ground the calcined tablet until it looked homogenous and then started the growth as before.

As shown in Fig. 2.6, the Laue pattern again was indicative of an unsuccessful growth in terms of growing a single crystal; however, the result was golden brown and shiny as $\mathrm{BaBiO}_{3}$ has to be 7,62 . We concluded that we are on the right path and perhaps we have to modify this "pre-reaction" step further. 

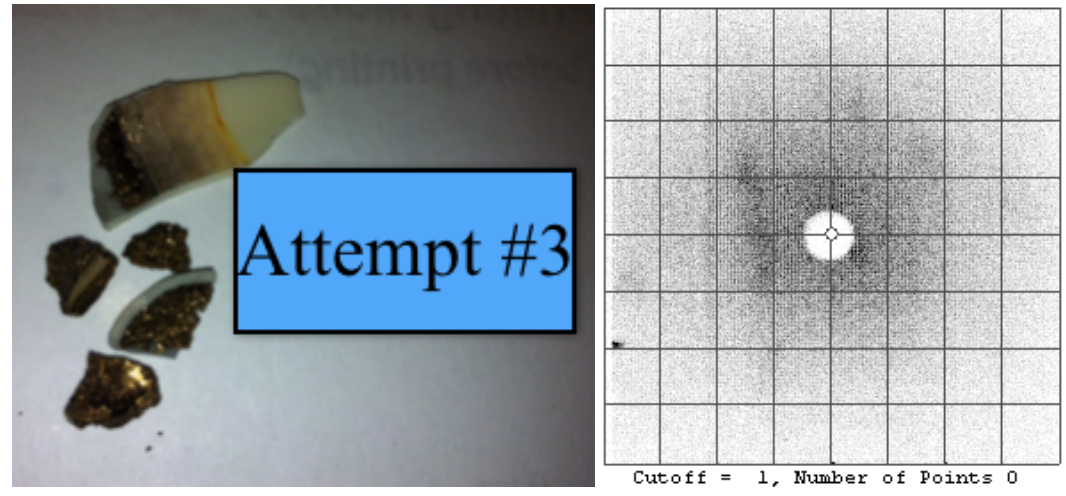

Figure 2.6: Third growth attempt and its Laue-gram. Here the previous procedure was repeated but the calcined powders was ground and homogenized before the melt. The result was golden brown as expected for $\mathrm{BaBiO}_{3}$ but not single crystal. (unsuccessful)

\section{Fourth Attempt}

From the previous attempt, we learned that the starting material has to be more homogeneous, and heating up the $\mathrm{Bi}_{2} \mathrm{O}_{3}$ and $\mathrm{BaCO}_{3}$ mixture at $800^{\circ}$ $\mathrm{C}$ for 5 hours, as suggested by the Ref. [9], does not give us that. Therefore, we decided to make sure we are starting with homogeneous single phase $\mathrm{BaBiO}_{3}$ powder before the melting step. It turned out this is a crucial step for a successful single crystal growth.

To achieve that, we again mixed powders of $\mathrm{BaCO}_{3}(99.9 \%)$ and $\mathrm{Bi}_{2} \mathrm{O}_{3}$ $(99.9 \%)$ in the stoichiometric molar ratio of $2: 1$ (10.000 $\mathrm{g}$ and $11.806 \mathrm{~g}$, respectively) and ground them patiently. The mixture then was sintered for 20 hours at $800^{\circ} \mathrm{C}$. Then we took out the calcined tablet, broke it and ground it thoroughly before putting it back in the furnace at $800^{\circ} \mathrm{C}$ for another 20 hours.

The final result did not look black and patchy anymore but all shimmery brown which was desired. It was again ground finely and was confirmed 
qualitatively by X-Ray Diffraction (XRD) test to be monoclinic $\mathrm{BaBiO}_{3}$ powder to make sure the solid-state reaction between the starting powders was successful prior to the melt procedure. A more detailed and quantitative analysis was intended to be carried out on the final crystals.

The measurement was performed using Philips X'pert X-ray diffractometer at Advanced Materials Process Engineering Laboratory (AMPEL), UBC. The XRD result is shown in Fig. 2.7 and is compared to the corresponding simulated pattern. The simulation has been done using the Vesta program with the lattice parameters given in table 2.1 from the Sleight and Cox paper $2 . \mathrm{BaBiO}_{3}$ powder XRD pattern is quite complicated with more than 100 peaks in the $20^{\circ}<2 \theta<60^{\circ}$ range. These peaks are given explicitly in Appendix $\mathrm{A}$ for the same calculation. $2 \theta$ is the angle between the incident beam and the diffracted beam. Physics of an XRD experiment is explained in more detail in section 2.3 .2 where the single crystal XRD results on the final crystals are presented.

\begin{tabular}{|c|c|c|c|c|c|}
\hline$a$ & $b$ & $c$ & $\alpha$ & $\beta$ & $\gamma$ \\
\hline $6.181 \AA$ & $6.136 \AA$ & $8.670 \AA$ & $90.000^{\circ}$ & $90.170^{\circ}$ & $90.000^{\circ}$ \\
\hline
\end{tabular}

Table 2.1: $\mathrm{BaBiO}_{3}$ lattice parameters in $I 2 / m$ symmetry taken from Ref. [2] and used to simulate the powder diffraction pattern in Fig. 2.7. 


\subsection{Single Crystal Growth Procedure}
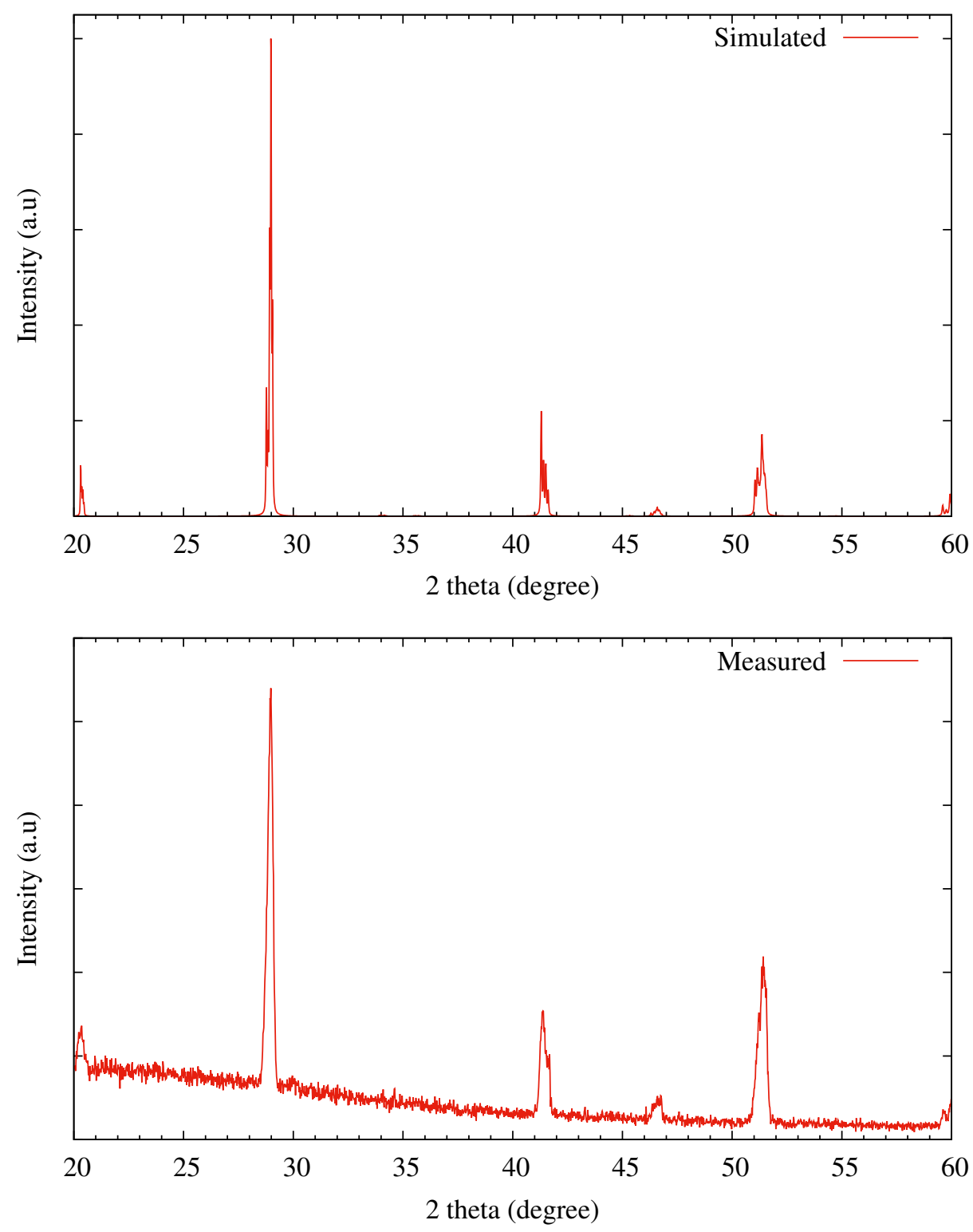

Figure 2.7: $\quad$ Simulated (top) and measured (bottom) $\mathrm{BaBiO}_{3}$ powder diffraction patterns with $\mathrm{Cu}-\mathrm{K} \alpha$ X-ray source.

After synthesizing the $\mathrm{BaBiO}_{3}$ powder, we started the melt growth with the following rates and temperatures: 


$$
\begin{aligned}
& 25^{\circ} \mathrm{C} \stackrel{180^{\circ} \mathrm{C} / h}{\Longrightarrow} 955^{\circ} \mathrm{C}(20 h) \stackrel{60^{\circ} \mathrm{C} / h}{\Longrightarrow} 1085^{\circ} \mathrm{C}(20 h) \\
& \stackrel{0.5^{\circ} \mathrm{C} / \mathrm{h}}{\Longrightarrow} 1015^{\circ} \mathrm{C}(10 h) \stackrel{300^{\circ} \mathrm{C} / h}{\Longrightarrow} 25^{\circ} \mathrm{C}
\end{aligned}
$$

The temperatures and steps were also slightly modified compared to the reference temperature profile [9]: The cooling rate was decreased from $4^{\circ} \mathrm{C} / \mathrm{h}$ between the temperatures $1100^{\circ} \mathrm{C}$ and $750^{\circ} \mathrm{C}$ to $0.5^{\circ} \mathrm{C} / \mathrm{h}$ between $1085^{\circ} \mathrm{C}$ to $1015^{\circ} \mathrm{C}$ and the stabilization constant-temperature intervals was increased in time in order to allow for growing bigger single crystal domains. The steps are shown in Fig. 2.8. This procedure takes about 190 hours in addition to the two 20-hour prior solid-state reactions.

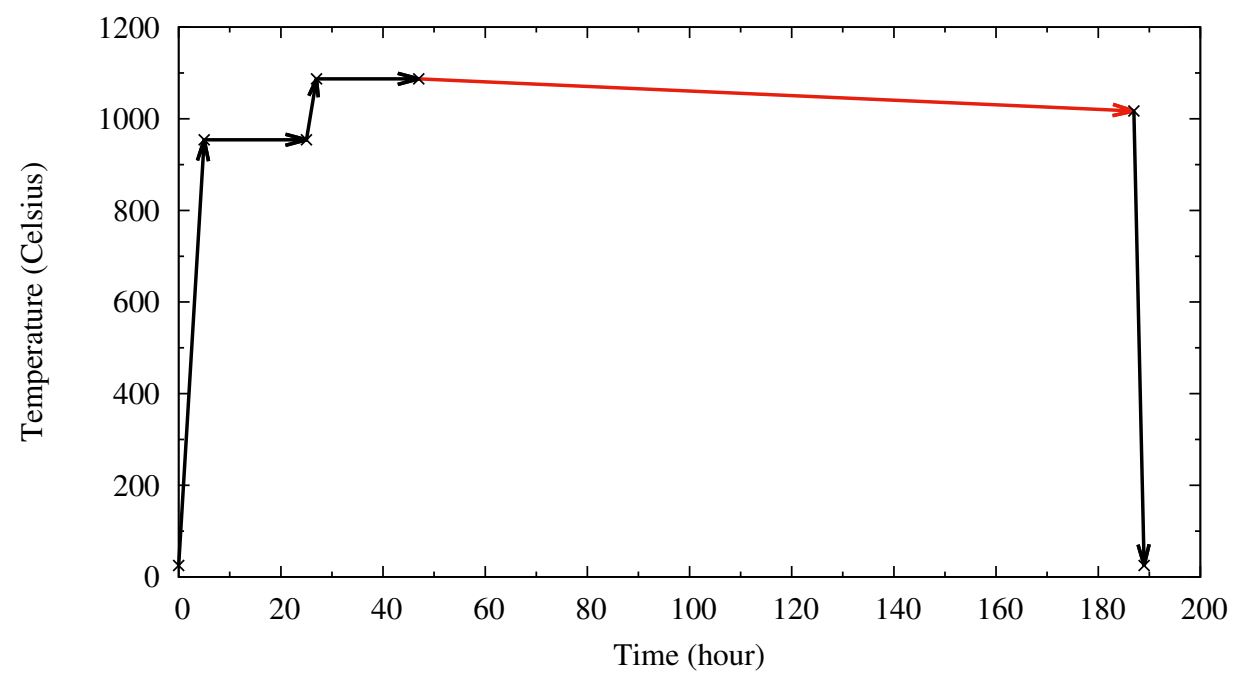

Figure 2.8: The thermal process for the $\mathrm{BaBiO}_{3}$ singe crystal melting growth. The red arrow indicates the slow cooling step from above the melting point to the below.

With the above procedure, we succeeded to grow $1 \times 1 \mathrm{~mm}^{2}$ plates of $\mathrm{BaBiO}_{3}$ single crystals from the surface as was confirmed by the Laue diffraction test. The result is shown in Fig. 2.9.

From the observation that the single crystal growth had only happened at the surface, we concluded that in order to get bigger single crystals we need to increase the surface area by using a wider crucible to enhance the oxygen exposure which was essential for a successful growth. This was the change we considered for the next growth. 

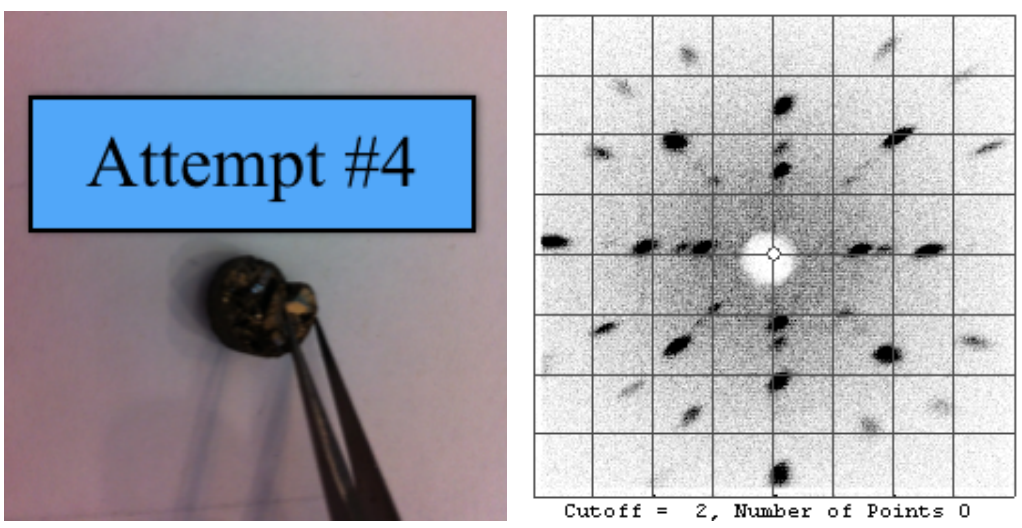

Figure 2.9: Fourth growth attempt and its Laue-gram. The melting procedure was performed on $\mathrm{BaBiO}_{3}$ powder which had been synthesized in advance through solid-state reaction. The result was $1 \times 1 \mathrm{~mm}^{2}$ plates of $\mathrm{BaBiO}_{3}$ single crystals. ( successful).

\section{Final Attempt}

We learned so far that in order to grow $\mathrm{BaBiO}_{3}$ single crystals through a melting or self-flux procedure we must start with the $\mathrm{BaBiO}_{3}$ powder. Moreover, to synthesize the powder, we should mix and grind stoichiometric ratio of $\mathrm{BaCO}_{3}$ and $\mathrm{Bi}_{2} \mathrm{O}_{3}$ powders and sinter the mixture twice at $800^{\circ} \mathrm{C}$ for 20 hours each. We need to grind the mixture thoroughly in between, too.

We also learned that having a temperature gradient does not help with the result since the crystals form on the surface. That is why this time, we followed the above procedure, as described in the previous attempt, and only changed the size of the crucible from a $10 \mathrm{~mm}$ OD to a $25 \mathrm{~mm}$ OD to increase the oxygen (air) exposure. As shown in Fig. 2.10, this way we managed to grow $\mathrm{BaBiO}_{3}$ single crystals of sizes up to $3 \times 3 \times 1 \mathrm{~mm}^{3}$. 


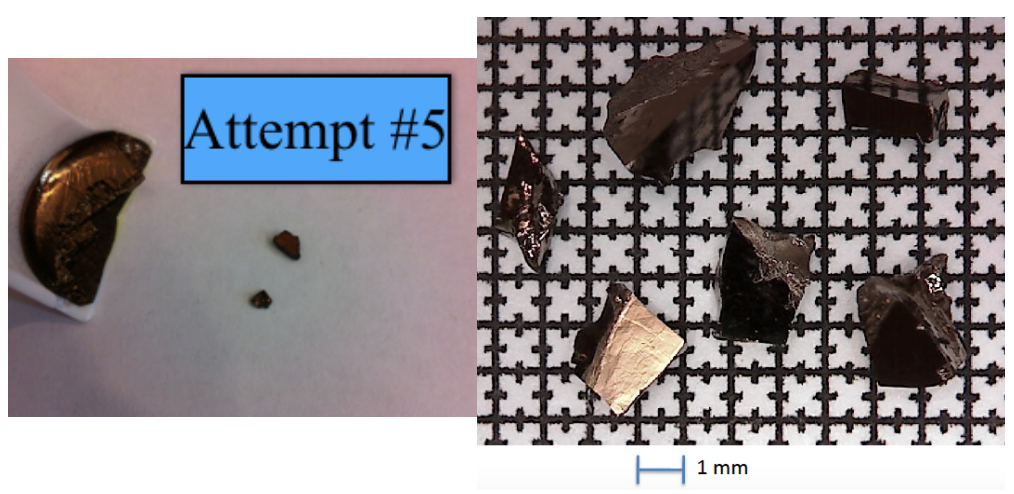

Figure 2.10: Fifth (final) growth attempt. Increasing the melt surface area by using a wider crucible (25 mm OD) resulted in growing bigger single crystals. (successful)
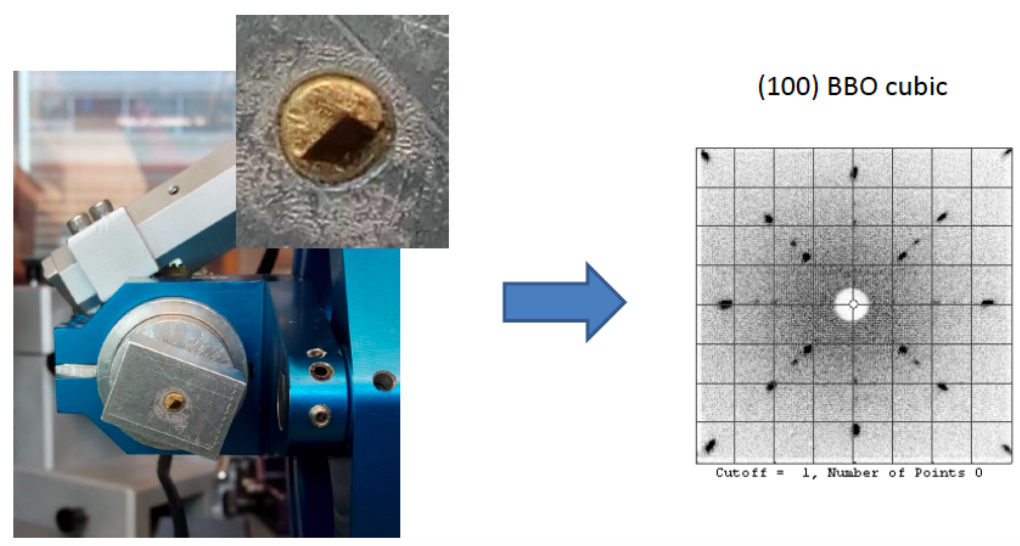

Figure 2.11: X-ray back-reflection Laue-gram of the grown crystal with the $\mathrm{x}$-rays oriented along the [100] crystallographic direction in a cubic unit cell. 


\subsection{Characterization}

Previously, it was explained how we developed a routine to grow $\mathrm{BaBiO}_{3}$ single crystals of sizes up to $3 \times 3 \times 1 \mathrm{~mm}^{3}$. The starting powder was confirmed by XRD to be $\mathrm{BaBiO}_{3}$, and the final crystals were confirmed by Laue diffraction to be single crystals. After these preliminary tests, we characterize the crystals in more detail using X-ray Photoemission Spectroscopy (XPS), single crystal XRD, and resistivity measurements to study the chemical composition, their crystal structure, and their transport properties, respectively.

\subsubsection{X-ray Photoemission Spectroscopy}

In order to find the chemical composition of the grown single crystals, we used XPS. Through XPS, we measure the number of electrons ejected from the surface when irradiated with a beam of x-rays. The measured spectrum includes a background signal and a set of resonance peaks. Each element in the few nanometers of the sample surface produces a set of peaks at characteristic binding energies corresponding to the electron configuration of the atom. The physics of this process is explained in more detail in chapter 4 .

In XPS when a sample is homogeneous the number of detected photoelectrons per second in a specific peak is determined by:

$$
I=n f \sigma \theta y \lambda A T
$$

where $n$ is the number of atoms of the element per volume unit, $f$ is the $\mathrm{x}$-ray flux, $\sigma$ is the photoelectric cross section for the given atomic orbital, $\theta$ is the angular efficiency factor, $y$ is the photoelectric process efficiency factor, $\lambda$ is the mean free path of the photoelectrons in the sample, $A$ is the area of the sample from which the photoelectrons are emitted, and $T$ is the detection efficiency factor.

$$
n=\frac{I}{f \sigma \theta y \lambda A T} \rightarrow \frac{n_{1}}{n_{2}}=\frac{I_{1} / S_{1}}{I_{2} / S_{2}}
$$

where $S$ is called atomic sensitivity factor. This equation implies the number of electrons recorded in a given state is proportional to the number of the corresponding atoms. It makes XPS a suitable tool to find the chemical composition. Finding the area enclosed by each characteristic peak is the primary way to find the number of excited electrons and hence the 


\subsection{Characterization}

corresponding chemical composition when the appropriate sensitivity factors are given 15 . There are many XPS softwares available that calculate these chemical compositions for a given spectrum. We used CasaXPS for this purpose.

As will be discussed in more detail in chapter 4, an XPS spectrum consists of some intrinsic peaks sitting on a background signal.

This "background" intensity has to be removed from the spectrum prior to finding the chemical composition of the material. A very crude approach to generate this background uses a simple linear approximation by interpolating the intensities at either end of an energy interval as:

$$
L(E)=I_{1} \frac{\left(E_{2}-E\right)}{\left(E_{2}-E_{1}\right)}+I_{2} \frac{\left(E-E_{1}\right)}{\left(E_{2}-E_{1}\right)}
$$

where $E_{1}$ and $E_{2}$ are ends of an energy interval and $I_{1}$ and $I_{2}$ are their corresponding intensities. However, the background shape often depends on the material and the energy. Therefore, a more complex approach is required to account for it such as the Shirley algorithm [63. It is an iterative approach to construct a background by using information about the spectrum.

After irradiating the sample with x-rays, the excited electrons may undergo inelastic collisions within the sample which alter their kinetic energies. They also can produce a multitude of low energy electron-hole excitations or so called secondary electrons. These two processes produce an energy-loss background plus a background due to the secondary electrons.

Because of the broad range in the energy loss and the secondary backgrounds a seemingly smooth background results at energies well below the peaks in the XPS spectrum. This background signal however only starts at the energies corresponding to zero-loss photoelectrons with kinetic energies less than the photoelectrons' kinetic energy. We can describe the variation of their intensities within a peak by composing a peak out of a continuous series of delta functions each one being followed at a lower kinetic energy by a step function of a height proportional to the intensity of that delta function. The total background within the peak shape is the integral over energy of those delta functions and the stepwise contribution which results in an "S"-like shape background as shown in Fig. 2.12, This procedure is called the Shirley background procedure.

The $n$-th iteration of the constructed Shirley background $\left(B_{n}(E)\right)$ and the $n$-th scattering factor $\left(\kappa_{n}\right)$ are given by: 


$$
\begin{array}{r}
B_{1}(E)=\kappa_{1} \int_{E}^{E_{1}} d E^{\prime}\left(I\left(E^{\prime}-I_{1}\right),\right. \\
B_{2}(E)=\kappa_{2} \int_{E}^{E_{1}} d E^{\prime}\left(I\left(E^{\prime}-I_{1}-B_{1}\left(E^{\prime}\right)\right)\right. \\
B_{n}(E)=\kappa_{n} \int_{E}^{E_{1}} d E^{\prime}\left(I\left(E^{\prime}-I_{1}-B_{n-1}\left(E^{\prime}\right)\right)\right.
\end{array} .
$$

and

$$
\kappa_{n}=\frac{I_{2}-I_{1}}{\int_{E_{2}}^{E_{1}} d E^{\prime}\left(I\left(E^{\prime}\right)-I_{1}-B_{n-1}\left(E^{\prime}\right)\right)}
$$

The binding energies $E_{1}$ and $E_{2}$ and the intensities $I_{1}$ and $I_{2}$ correspond to the two selected points, one at an energy above and one below the peak, as shown in Fig. 2.12 for a carbon $1 s$ spectrum. Note $E_{2}$ is smaller than $E_{1}$. Equation 2.6 guaranties at each iteration the scattering factor $\kappa$ is chosen so that the background goes through the other point $\left(E_{2}, I_{2}\right)$. This iterative process continues until the background value, $B_{n}$, converge[10]. 

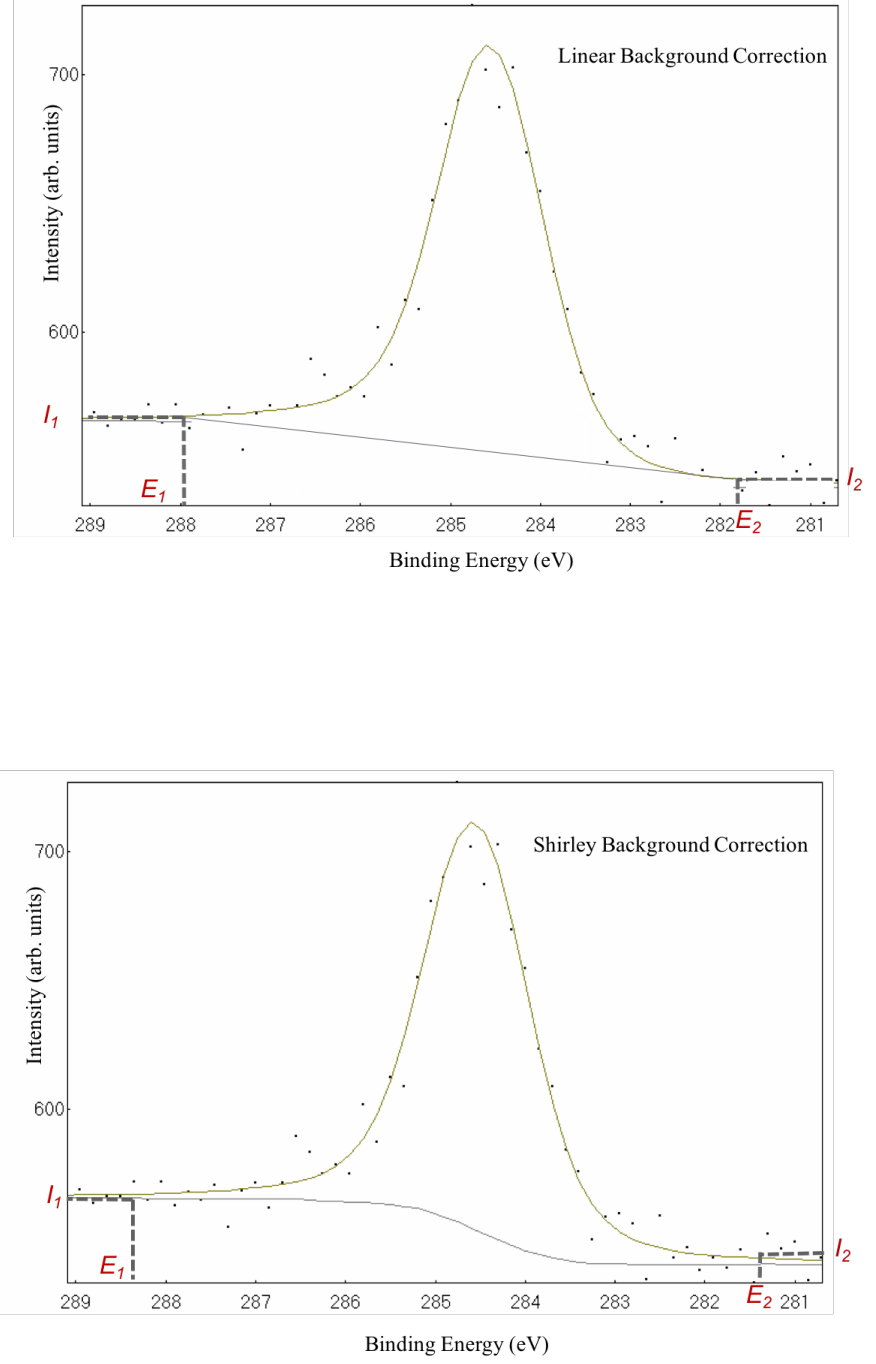

Figure 2.12: A carbon $1 s$ x-ray photoemission spectrum with the background signal corrected using the top: linear and bottom: Shirley algorithms. The figures are taken from Ref. [10] and they have been modified slightly by adding the labels. 


\subsection{Characterization}

There are many commercial software packages available to construct a background intensity using different algorithms and subtract it from the XPS spectrum. We picked Shirley background algorithm and used the CasaXPS software for this purpose.

The XPS measurements are performed using an Omicron Multi-probe MXPS machine with an energy resolution of $0.4 \mathrm{eV}$ at the Surface Science Facility of the REIXS beam-line at the Canadian Light Source (CLS). The system is shown in Fig. 2.13 which consists of two sources and one analyzer:

- Sources: XM 1000 and DAR 400. XM1000 generates monochromatic x-ray with $\mathrm{Al} K \alpha(\mathrm{h} \nu=1486.6 \mathrm{eV})$ which is used for our experiments. DAR 400 produces none-monochromatic x-ray with $\mathrm{Mg} \mathrm{K} \alpha$ (h $\nu=$ $1253.6 \mathrm{eV})$ and $\mathrm{Al} \mathrm{K} \alpha(\mathrm{h} \nu=1486.6 \mathrm{eV})$ anodes. The diameter of the monochromatic x-ray beam is $\leq 0.75 \mathrm{~mm}$.

- Analyzer: EA 125 SPHERA. It is a hemispherical analyzer which is mounted at $37^{\circ}$ from the horizontal and $83^{\circ}$ from the x-ray monochromator.

The crystal has been efficiently cleaned to remove surface contamination using the cleaning procedure described in chapter 4 .

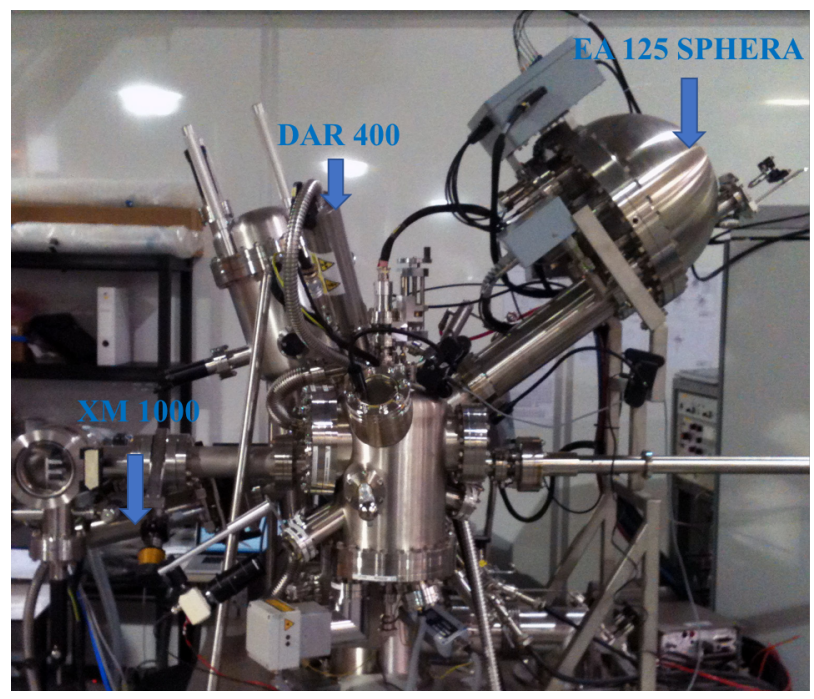

Figure 2.13: Omicron Multi-probe MXPS system at the Surface Science Facility of the REIXS beam-line at the Canadian Light Source (CLS) 
The XPS result is shown in Fig. 2.14 and the corresponding atomic ratios are given in table 2.2. The atomic ratios are calculated by finding the enclosed area under the $\mathrm{Ba} 3 d 5 / 2, \mathrm{Bi} 4 d 5 / 2$, and $\mathrm{O} 1 s$ peaks and their corresponding relative sensitivity factors.

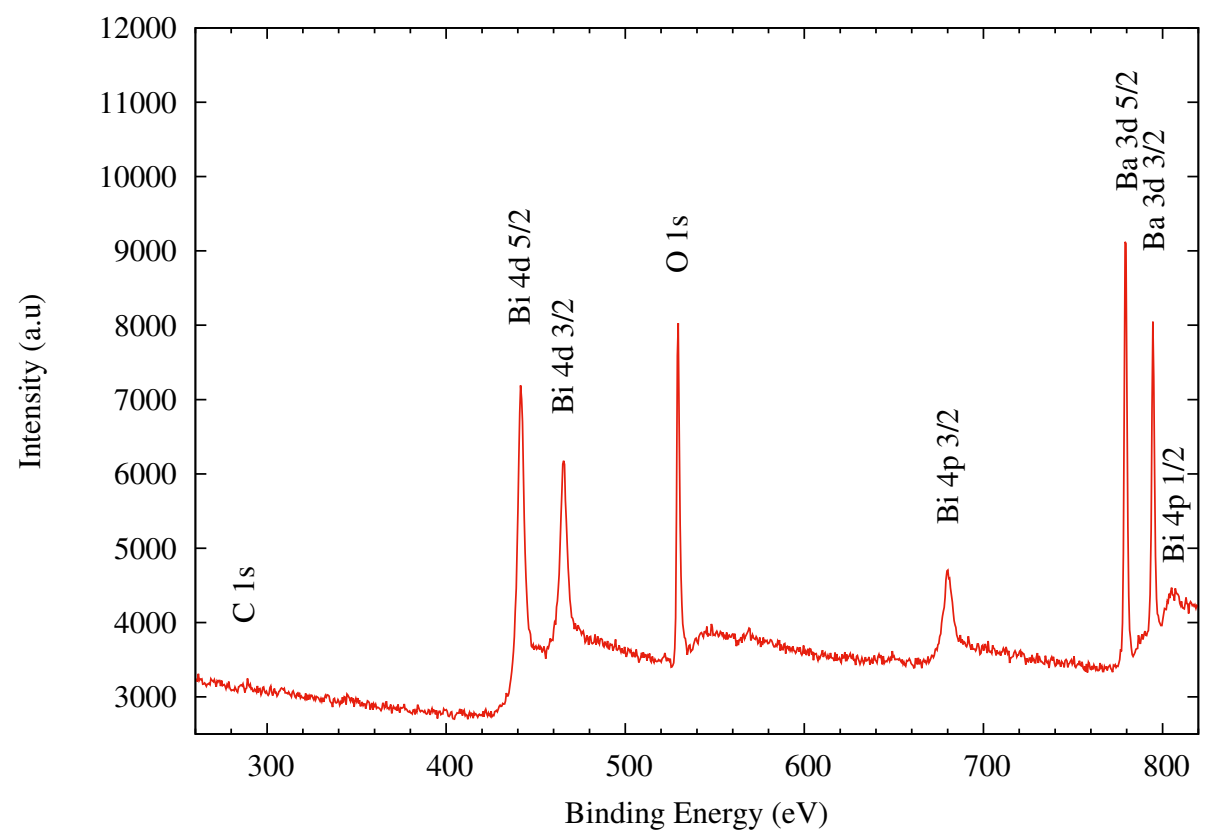

Figure 2.14: X-ray photoemission spectrum of $\mathrm{BaBiO}_{3}$ single crystal at room temperature

\begin{tabular}{|c|c|}
\hline Element & At $\%$ \\
\hline \hline $\mathrm{Ba}$ & $19 \pm 1$ \\
\hline $\mathrm{Bi}$ & $19 \pm 1$ \\
\hline $\mathrm{O}$ & $62 \pm 3$ \\
\hline
\end{tabular}

Table 2.2: The atomic ratios of the grown single crystal given by the XPS measurement (Fig. 2.14 ). The accuracy of the results derived from this technique (described in the text) is within $5 \%$. 


\subsection{Characterization}

\subsubsection{Ray Diffraction}

In real space, crystalline solids consist of series of planes of regularly spaced atoms separated by the distance $d$. When a beam of light hits these planes, there is a strong constructive interference of the reflected beams from the surface and the various layers if the path difference is integer multiple of the incident beam's wavelength. As explained below, this results in a formulated condition known as Bragg's law.

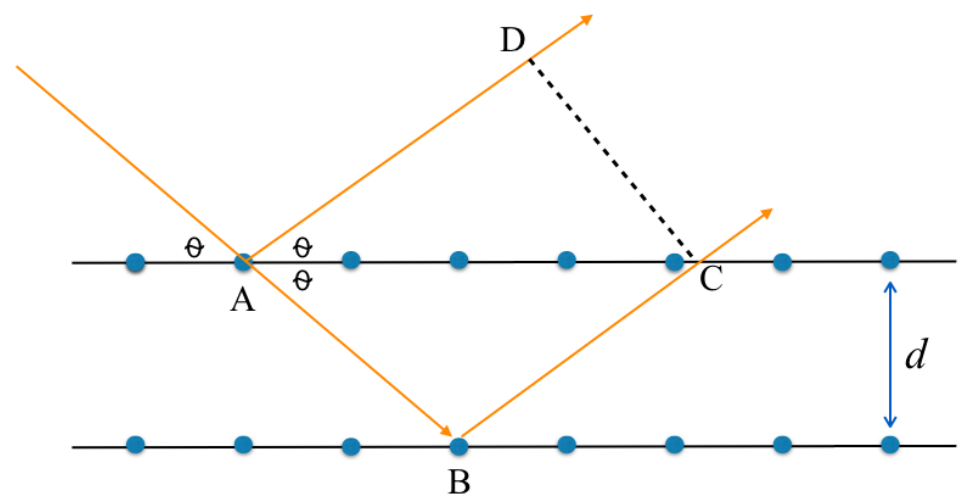

Figure 2.15: A schematic representation of a two-dimensional crystal lattice. An incident beam hitting the crystal undergoes a constructive (in phase) diffraction when the path difference is equal to an integral multiple of the wavelength: $n \lambda=2 d \sin \theta$. (Bragg's law)

As illustrated in Fig. 2.15, when a beam of rays hits a crystal, some reflect along $\mathrm{AD}$ by the first plane and some transmit and get reflected by the second plane along BC. The path difference between these two equals:

$$
\text { path difference }=(\mathrm{AB}+\mathrm{BC})-(\mathrm{AD})
$$

These beams will undergo constructive interference, if and only if:

$$
\text { path difference }=n \lambda,
$$

where, $n$ is a positive integer and $\lambda$ is wavelength of the incident wave. Based on a simple trigonometry in Fig. 2.15: 


$$
\begin{array}{r}
\mathrm{AB}=\mathrm{BC}=\frac{d}{\sin \theta} \\
\mathrm{AC}=\frac{2 d}{\tan \theta} \\
\mathrm{AD}=\mathrm{AC} \cos \theta \Rightarrow n \lambda=2 d \sin \theta
\end{array}
$$

For each lattice system, the inter-planar distance $d$ can be expressed in terms of the Miller indices $(h, k, l)$ for each set of planes. For the simplest

case of a cubic lattice with the lattice parameter of $a: \frac{1}{d^{2}}=\frac{h^{2}+k^{2}+l^{2}}{a^{2}}$, while for a more complicated case of a monoclinic system with lattice parameters $a, b, c$ and $\beta$ it is given by:

$$
\begin{array}{r}
\frac{1}{d_{\text {monoclinic }}^{2}=\frac{h^{2}}{a^{2} \sin ^{2} \beta}+\frac{k^{2}}{b^{2}}}+\frac{l^{2}}{c^{2} \sin ^{2} \beta} \\
+\frac{2 h l \cos \beta}{a c \sin ^{2} \beta}
\end{array}
$$

The most frequently used type of XRD measurement is a $2 \theta-\omega$ scan in which the $2 \theta$ axis is moved twice the rate of the $\omega$ axis. In a $2 \theta-\omega$ scan, the intensity of the diffracted beam is recorded and plotted as a function of $2 \theta$. $\omega$ is the angle between the incident beam and the sample surface, and $2 \theta$ is the angle between the incident beam and the diffracted beam. The resulting spectrum consists of a set of peaks at some specific angles. These peaks correspond to the above constructive Bragg reflections. Thus, with having the incident angles $(\theta)$, and the wavelength $(\lambda), d$ and subsequently the sample's lattice parameters can be found.

\section{Lattice Parameters}

After performing an XPS measurement to find the chemical composition of the crystals and a preliminary Laue diffraction test, we studied the crystal's structure in more detail using single crystal X-Ray Diffraction (XRD) technique. These measurements were performed at the X-Ray Crystallography facility of the UBC Chemistry department using Bruker APEX DUO single crystal diffractometer, equipped with a TRIUMPH monochromatic Mo K $\alpha$ radiation $(\lambda=0.71073 \AA)$. The cell was indexed using four scan sets each consisting of 24 images (96 in total).

As shown in table 2.3, the crystal's lattice parameters were measured at two different temperatures. The crystal was found to be of monoclinic $\mathrm{P} 2 / \mathrm{m}$ 


\begin{tabular}{|c|c|c|}
\hline \multicolumn{3}{|c|}{ Monoclinic $P 2 / m(\mathrm{~T}=90 \mathrm{~K})$} \\
\hline \hline$a=6.1728 \AA$ & $b=6.1452 \AA$ & $c=8.6926 \AA$ \\
\hline$\alpha=90.000^{\circ}$ & $\beta=90.0357^{\circ}$ & $\gamma=90.000^{\circ}$ \\
\hline \hline \multicolumn{3}{|c|}{ Monoclinic $12 / m(\mathrm{~T}=210 \mathrm{~K})$} \\
\hline \hline$a=6.1597 \AA$ & $b=6.1537 \AA$ & $c=8.6985 \AA$ \\
\hline$\alpha=90.000^{\circ}$ & $\beta=90.1333^{\circ}$ & $\gamma=90.000^{\circ}$ \\
\hline
\end{tabular}

Table 2.3: $\mathrm{BaBiO}_{3}$ single crystal lattice parameters measured at temperatures $90 \mathrm{~K}$ and $210 \mathrm{~K}$.

and monoclinic $I 2 / \mathrm{m}$ structure at $T=90 \mathrm{~K}$ and $T=210 \mathrm{~K}$, respectively. These results are in agreement with the previously published reports [2].

\section{Twinning}

As described in chapter $1, \mathrm{BaBiO}_{3}$ undergoes multiple structural transitions with temperature [2]. Therefore, in growing the crystal by cooling down the $\mathrm{BaBiO}_{3}$ melt at $1085^{\circ} \mathrm{C}$ to room temperature, it has to go through cubic to rhombohedral to monoclinic transitions. Studies show these types of structural transitions can cause multi-domain microstructures resulting in a twinned crystal [7, 45]. Therefore, we decided to study the crystal's structure in more detail to find out whether the crystal is twinned and if so, to what extent.

These measurements were done at AMPEL, UBC using Philips X'pert X-ray diffractometer with a $\mathrm{Cu} \mathrm{K}_{\alpha}$ source $(\lambda=1.54059 \AA)$. These measurements (and the following resistivity measurements) were lead and mainly carried out by Oleksandr Foyevtsov from our group. We started with a $\theta-2 \theta$ scan on a single crystal. The XRD spectrum and the corresponding Bragg reflections are shown in Fig. 2.16. The reflection coordinates are given in monoclinic notation and as expected from the previous Laue indexing measurement (Fig. 2.11) the main phase is the (110) family in monoclinic or (100) in cubic notation.

However, in a more detailed measurement it was seen that there are some contributions from another orientation of (001) monoclinic family. As shown in Appendix A these reflections are very close to the other ones; thus, it is difficult to distinguish and quantify their contributions in a $\theta-2 \theta$ scan. To 
achieve that, we performed high-resolution Reciprocal Scan Mapping (RSM) method around each Bragg reflection.

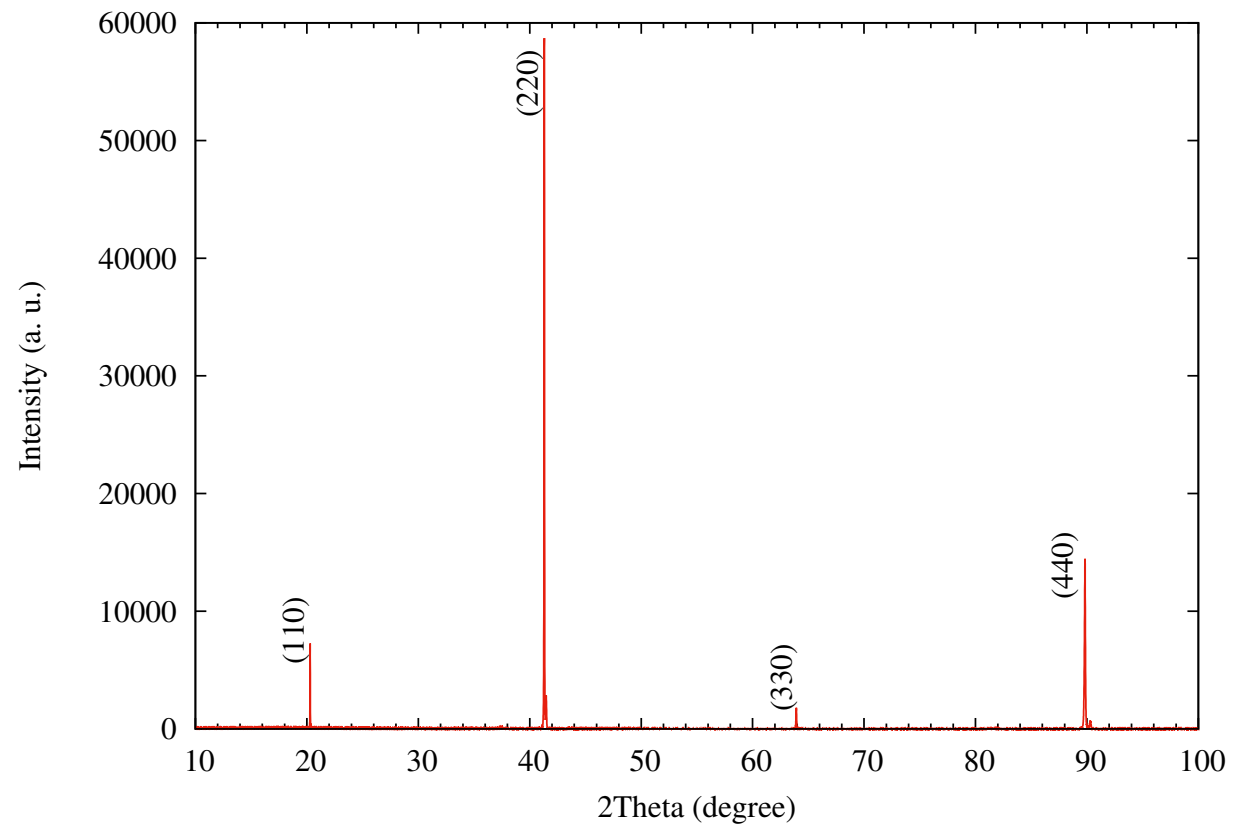

Figure 2.16: X-Ray Diffraction pattern on a grown $\mathrm{BaBiO}_{3}$ single crystal with the "as grown" surface facing.

The Bragg diffraction condition (Eq. 2.9) can alternatively be expressed in the reciprocal space or Laue formulation. Reciprocal lattice represents the Fourier transform of the lattice in the real space. The axis vectors of a reciprocal lattice, $b_{1}, b_{2}, b_{3}$, are related to the axis vectors of its real space lattice, $a_{1}, a_{2}, a_{3}$, via:

$$
\begin{aligned}
& \mathbf{b}_{1}=2 \pi \frac{\mathbf{a}_{2} \times \mathbf{a}_{3}}{\mathbf{a}_{1} \cdot\left(\mathbf{a}_{2} \times \mathbf{a}_{3}\right)} \\
& \mathbf{b}_{2}=2 \pi \frac{\mathbf{a} 3 \times \mathbf{\mathbf { a } _ { 1 }}}{\mathbf{\mathbf { a } _ { 2 }} \cdot\left(\mathbf{\mathbf { a } _ { 3 } \times \mathbf { a } _ { 1 } )}\right.} \\
& \mathbf{b}_{3}=2 \pi \frac{\mathbf{a}_{1} \times \mathbf{a}_{2}}{\mathbf{a}_{3} \cdot\left(\mathbf{a}_{1} \times \mathbf{a}_{2}\right)}
\end{aligned}
$$

Then it can be shown that the Bragg condition is equivalent to: 


$$
Q=\Delta q=G
$$

where $Q=\Delta q=q^{\prime}-q$ is the scattering vector and $\mathbf{G}=k_{1} \mathbf{b}_{\mathbf{1}}+k_{2} \mathbf{b}_{\mathbf{2}}+k_{3} \mathbf{b}_{\mathbf{3}}$ is a reciprocal lattice vector. Each point in a reciprocal space corresponds to a set of planes in its real space. In a reciprocal space x-ray diffraction measurement, intensity is plotted against the scattering vector.

In a coplanar diffraction geometry, the sample surface normal and the incident and the exit wave vectors all exist in the same plane. Most commercially available x-ray diffraction instruments perform in this geometry. In this case of geometry, two angular variables are sufficient to scan the accessible scattering vector plane $Q_{x}-Q_{y}$. The relevant angles in this geometry are depicted in Fig. 2.19. The components of scattering vector $Q$ and the experimental angles are related to each other by [64]:

$$
\begin{aligned}
Q_{x} & =\frac{2 \pi}{\lambda}\left(\cos \alpha_{f}-\cos \alpha_{i}\right) \\
Q_{y} & =\frac{2 \pi}{\lambda}\left(\sin \alpha_{f}+\sin \alpha_{i}\right)
\end{aligned}
$$

Or alternatively in terms of the scattering angles $2 \theta$ and $\phi$ which is related to $\omega$ via $\omega=\alpha_{i}+\phi$.

$$
\begin{array}{r}
Q_{x}=-|Q| \sin \phi=-\frac{2 \pi}{\lambda} \cdot 2 \sin \left(\frac{2 \theta}{2}\right) \sin \phi \\
Q_{y}=|Q| \cos \phi=\frac{2 \pi}{\lambda} \cdot 2 \sin \left(\frac{2 \theta}{2}\right) \cos \phi
\end{array}
$$




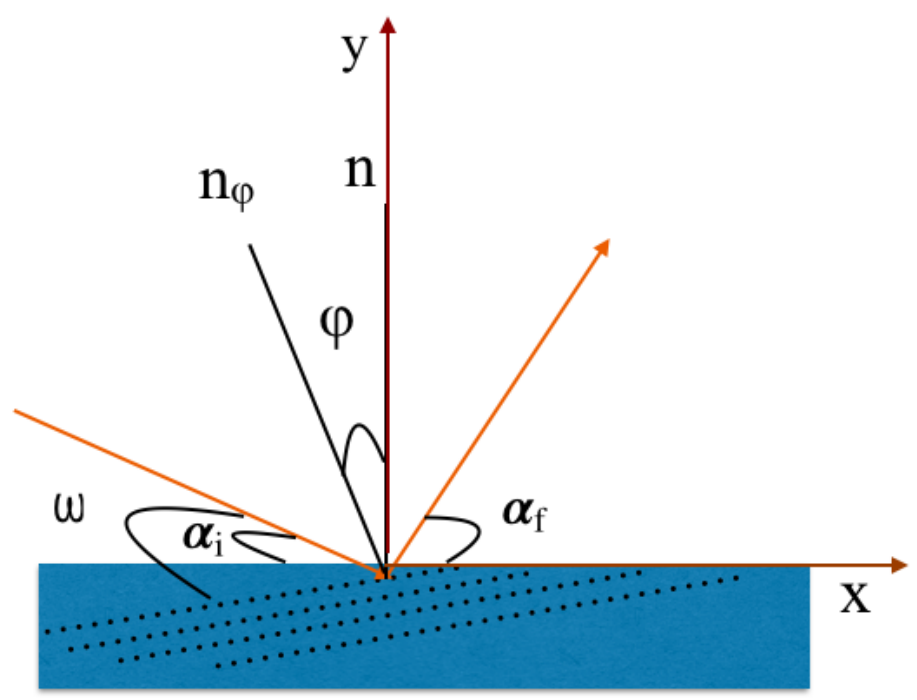

Figure 2.17: Schematic illustration of the coplanar diffraction geometry. $n$ is the normal to the sample surface and $n_{\phi}$ is the normal to the diffracting lattice planes. $\alpha_{i}$ and $\alpha_{f}$ are the angles of incident and exit. $\omega$ is the angle between the incident beam and the diffracting lattice planes, $\omega=\alpha_{i}+\phi$.

It is also relatively straightforward to do the inverse operation and find the diffractometer angles needed for a given scattering vector. Although, most modern diffractometers give the reciprocal space maps directly in the Q-space.

Our reciprocal space maps around each Bragg reflection are shown in Fig. 2.19. As can be seen, we have two main sets of constructive Bragg reflections corresponding to (110) and (001) families giving evidence to the existence of two crystal domains. Now in order to quantify this observation, the ratios of the two integrated intensities for each two reflections from experiment ,$r_{1}$, have been compared to the corresponding calculated intensity ratio for a powder x-ray diffraction pattern, $r_{2}$, or with the assumption that there is an equal amount of both phases in the crystal. The parameter $R$ is also defined as the ratio of these two parameters (Eq. 2.19). In table 2.4 these two are compared. 


$$
\begin{array}{r}
r_{1}=\frac{I_{(110)}^{\mathrm{exp}}}{I_{(002)}^{\mathrm{exp}}} \\
r_{2}=\frac{I_{(110)}^{\text {calc }}}{I_{(002)}^{\text {calc }}} ; \\
R_{(110) /(002)}=\frac{r_{1}}{r_{2}}
\end{array}
$$

If $R$ is equal to one, it means the crystal is 50/50 twinned or there is an equal amount of the both mentioned phases.

In table 2.4 these ratios are given for each map. As shown the mean value of $R$ is found to be 0.130 , which means the $2 \times 2 \times 1 \mathrm{~mm}^{3}$ crystal was $13 \%$ twinned with a standard deviation of 0.020 . 


\subsection{Characterization}
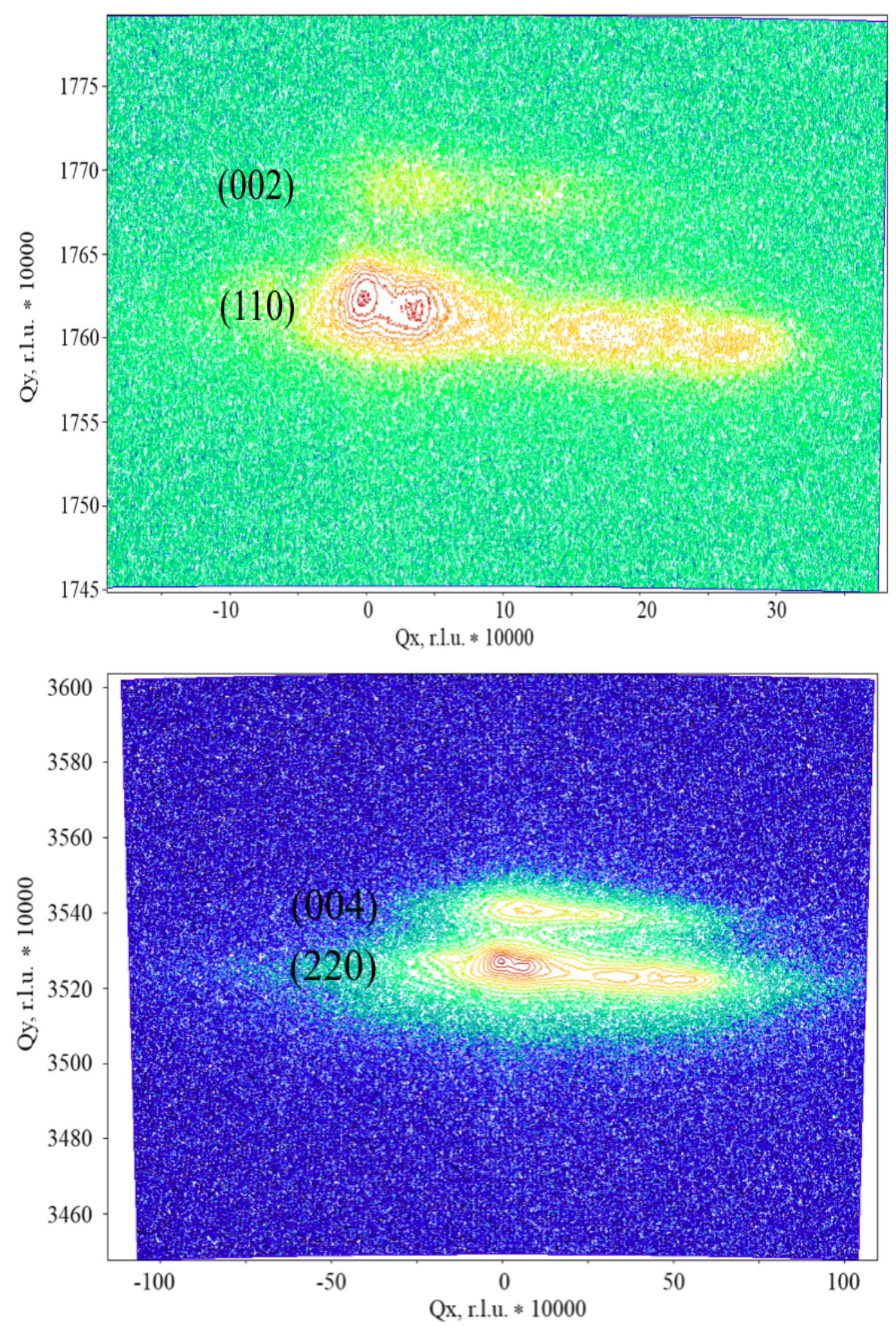

Figure 2.18: Reciprocal space maps of crystal $\mathrm{BaBiO}_{3}$ top: around (110) and (002) and bottom: around (220) and (004) Bragg diffractions. The axes are in reciprocal lattice unit (r.l.u) which is $\frac{\lambda}{2 d_{h k l}}$ where $\lambda=1.54059 \AA$. 


\subsection{Characterization}
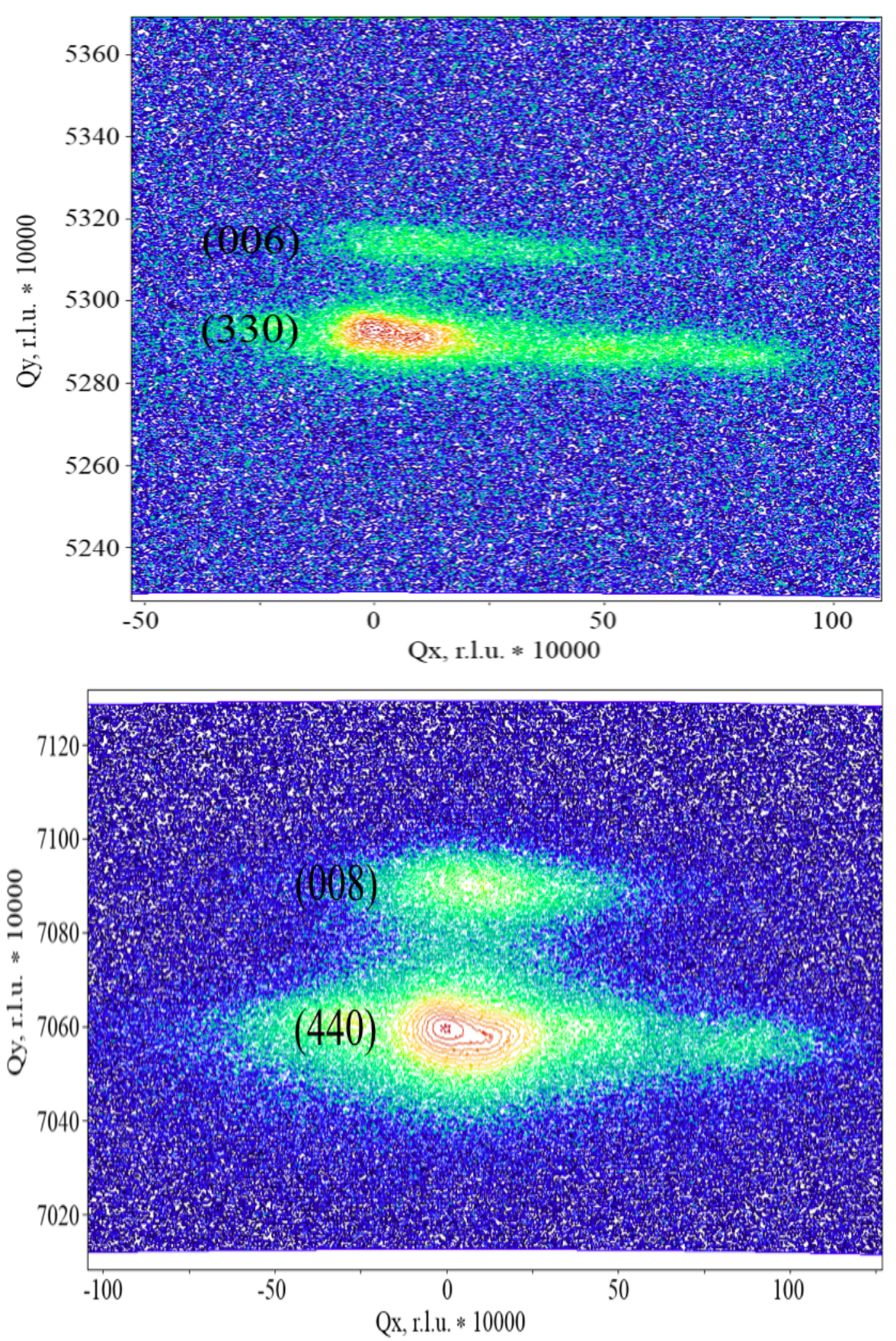

Figure 2.19: Reciprocal space maps of crystal $\mathrm{BaBiO}_{3}$ top: around (330) and (006) and bottom: around (440) and (008) Bragg diffractions. The axes are in reciprocal lattice unit (r.l.u) which is $\frac{\lambda}{2 d_{h k l}}$ where $\lambda=1.54059 \AA$. 


\subsection{Characterization}

\begin{tabular}{|c|c|}
\hline Bragg Reflection Pair & Measured/Simulated Intensity Ratio (R) \\
\hline \hline$(110) /(002)$ & 0.154 \\
\hline$(220) /(004)$ & 0.143 \\
\hline$(330) /(006)$ & 0.125 \\
\hline$(440) /(008)$ & 0.100 \\
\hline \hline Mean Value & 0.130 \\
\hline Standard Deviation & 0.020 \\
\hline
\end{tabular}

Table 2.4: The $\mathrm{R}$ value for different Bragg reflection pairs. $\mathrm{R}$ is defined in Eq. 2.19 and is indicative of contribution from different crystal domains. This result implies that the given crystal was $13 \%$ twinned.

\subsubsection{Resistivity}

We also performed temperature dependent resistivity measurements on selected single crystals of $\mathrm{BaBiO}_{3}$ using a lock-in technique in a four-probe configuration. The schematic representation of the experimental set up is shown in Fig. 2.20. The measurement was done as a function of temperature from $10 \mathrm{~K}$ to room temperature. Both direct wire bonding of Si-doped $\mathrm{Al}$ wire and the same wire attached with colloidal silver paste were used to make contacts. Both methods gave consistent results. The result is shown in Fig. 2.21.

The temperature dependence of resistivity is explained by the Arrhenius equation as:

$$
\rho \propto e^{\frac{E_{a}}{k_{B} T}}
$$

where $E_{a}$ is known as the activation energy. Therefore, one can find the activation energy by finding the slope of the $\log \rho$ versus $1 / T$ plot (Fig. 2.21). As shown, an abrupt change in the activation energy happens at temperature about $T_{c}=230 \mathrm{~K}$ from $E_{a}=207 \mathrm{meV}$ below $T_{c}$ to $E_{a}=143 \mathrm{meV}$ above. This result is consistent with the earlier published results $[7,37,65]$. 


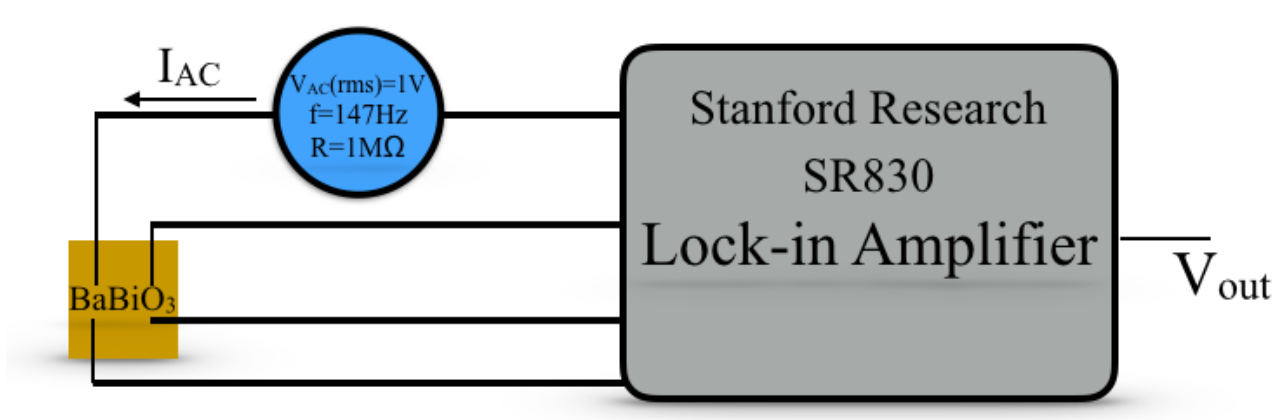

Figure 2.20: Schematic representation of the 4-probe resistivity measurement using a lock-in amplifier.

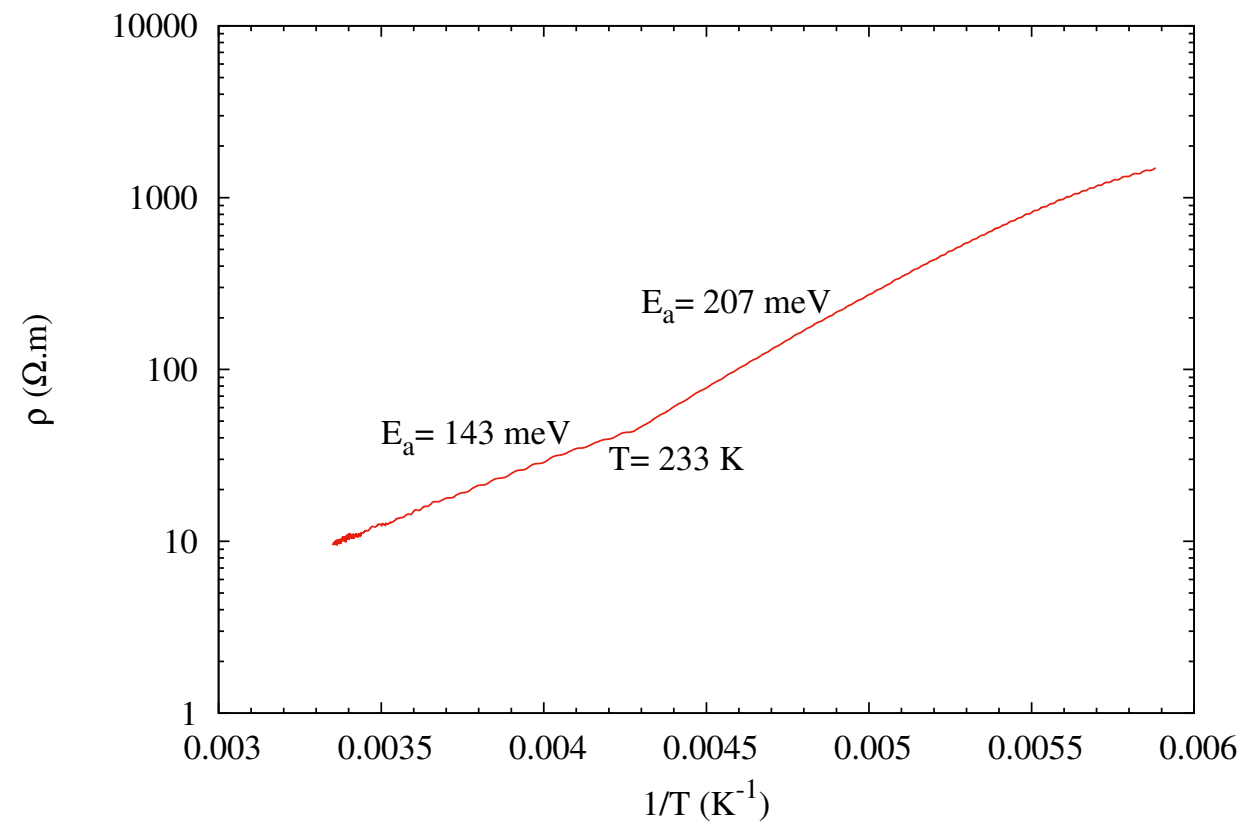

Figure 2.21: Resistivity measurement of $\mathrm{BaBiO}_{3}$ crystal. An abrupt change in slope, i.e. activation energy $\left(E_{a}\right)$, happens at $T=233 \mathrm{~K}$. 


\subsection{Conclusion}

\subsection{Conclusion}

We developed a routine to grow high-quality single crystals of $\mathrm{BaBiO}_{3}$ with sizes up to $2 \times 2 \times 1 \mathrm{~mm}^{3}$. It includes a two-step high-temperature solid state reaction followed by a congruent melting procedure. Using a two-step solid state reaction before the melt growth and also using a wide crucible (> $25 \mathrm{~mm}$ outer diameter) during the melt growth were found to be crucial in order to get reasonably big single crystals. Otherwise, the crystal would only form as very thin plates on the surface of the melt.

The crystals were characterized using XRD, XPS and found to be stoichiometric and monoclinic within the accuracy of these techniques. The crystal was found to be of monoclinic $P 2 / \mathrm{m}$ and monoclinic $I 2 / \mathrm{m}$ structure at $T=90 \mathrm{~K}$ and $T=210 \mathrm{~K}$, respectively. The lattice parameters at those temperatures were also found. Twinning was also studied on a given crystal by a detailed reciprocal space diffraction analysis and the crystal was found to be $13 \%$ twinned with two main domains. The temperature dependent electronic transport measurement was also performed on the crystal and two activation energies were found with a transition at $T=233 \mathrm{~K}$. These results are in agreement with the previously published reports. 


\section{Chapter 3}

\section{X-ray Absorption and Resonant X-ray Emission Spectroscopy}

In this chapter, we use x-ray absorption and resonant x-ray emission spectroscopy techniques to study the electronic structure of the single crystal $\mathrm{BaBiO}_{3}$. The measured crystal was grown as explained in chapter 2, The x-ray experiments are performed at the Canadian Light Source (CLS), and the results are compared to the prediction from density functional theory.

\subsection{Introduction}

After the discovery of x-rays by Rontgen [66, 67] in 1895, it took about two decades before the first x-ray absorption spectrum was observed by de Broglie in 1913 [68]. The core-level x-ray spectroscopy techniques such as $\mathrm{x}$-ray absorption and x-ray emission have come a long way since then by the remarkable progress made in both experimental and analytical capabilities. Resonant x-ray emission is often used as a powerful complementary technique to $\mathrm{x}$-ray absorption to investigate together the low energy scale valence and conduction bands of the material. The first theory of x-ray absorption fine structure was published in 1931 by Ralph Kronig which contained some of the basic concepts of its modern interpretation [69]. The other groundbreaking progress happened in 1971 when Sayers, Stern, and Lytle applied Fourier analysis to the theory of x-ray absorption where they managed to quantitatively determine structural parameters, such as bond distance and coordination number $[70,71]$.

Arguably, the main experimental development that allowed XAS+RXES to become a reliable and routine analytical tool was the availability of broadband synchrotron radiation sources starting modestly in the 1970s. Since then, these synchrotron radiation laboratories have grown enormously both in number and in capability. Synchrotron radiation offers significant benefits 


\subsection{Introduction}

in intensity/flux and experimental quality, along with the ability to select a specific light wavelength and polarization.

XAS involves exciting electrons from a core state to an empty state above the Fermi level induced by incident photons. It gives spectra of the x-ray absorption versus x-ray energy. At some specific energies, mainly depending on the atomic number of the absorbing atom, the absorption probability abruptly increases which is called an "edge". Each edge represents a different core electron binding energy and is named according to the principal quantum number of the excited electron: $\mathrm{K}$ for $n=1$, L for $n=2, \mathrm{M}$ for $n=3$, etc. Since the core level is at a rather sharp and well-defined energy characteristic of the element and its immediate surroundings, scanning through the x-ray energy provides information of these unoccupied states in the material.

Absorption near and immediately above an edge is due to the excitation of core electrons into bound states, in the region often called the Near Edge X-ray Absorption Fine Structure (NEXAFS) or X-ray Absorption Near Edge Structure (XANES). NEXAFS is rich in information about the electronic and magnetic structure of the absorbing atom as well as its surrounding symmetry and geometry. The bound states in this region are a result of the strong Coulomb interaction between the core-hole and the valence electron. They happen if this interaction is much larger than the bandwidth of the valence electrons due to the interatomic hoping. This is often the case in transition metal compounds as well as rare earth compounds. These bound or "excitonic" states represent the excited states of the atom or ion and the fine structure seen in these bound states together with the dipole selection rules in XAS provides direct information of the ground state occupation of the valence states [71].

Further above the edge, the core electron has sufficient energy to reach continuum states. This region shows a slow decay in the absorption rate versus energy, with superimposed oscillating structures due to scattering of the excited electron off neighbouring atoms. Studying this wide region of the spectra is known as Extended X-ray Absorption Fine Structure (EXAFS).

On the other hand, in an x-ray emission process, a core electron is excited by incident x-ray photons, and then an electron decays from an occupied valence band into the unoccupied core state emitting a photon. When the core electron is excited to the continuum, it is called Normal X-ray Emission Spectroscopy (NXES), and when the incident x-ray energy is tuned to resonate with the excitation edge of the core electron, it is called resonant x-ray emission(RXES). For this work, we use the oxygen K-edge NEXAFS+RXES to study our material of interest, $\mathrm{BaBiO}_{3}$. 


\subsection{Introduction}

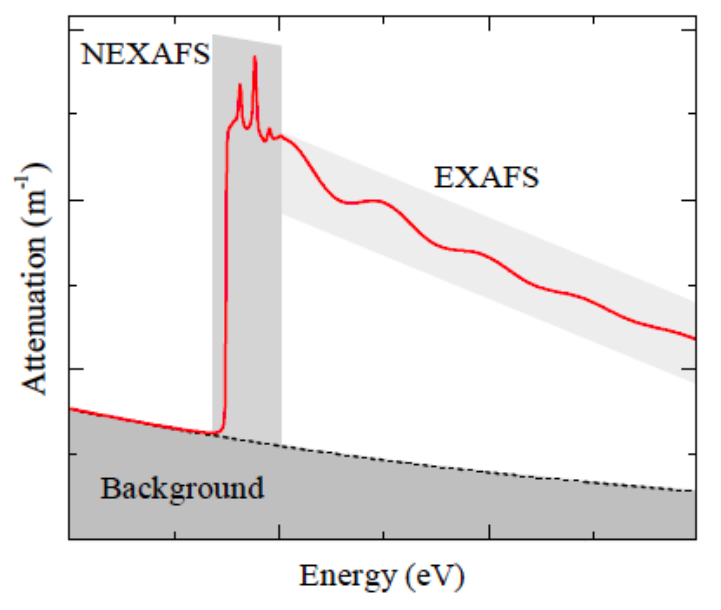

Figure 3.1: The three main regions of an x-ray absorption spectrum [11].

\subsubsection{Theory}

XAS involves exciting electrons by incident photons from a core state to an unoccupied state above the Fermi level. Therefore, it probes the unoccupied states and provides valuable information about the electronic structure of the material. Due to the localized character of the photon-induced excitation and the specific binding energy of the core electron, XAS is a site-selective technique.

In this section, we describe the interaction of x-rays with matter in a simple single-particle picture. The interaction is, in fact, much more complicated, but this simplified approach works well in giving insight to the underlying physics and derivation of the key dipole selection rules.

The Hamiltonian for electrons in an electromagnetic field can be expressed as :

$$
H=\frac{1}{2 m}\left(\vec{p}+\frac{e}{c} \vec{A}\right)^{2}+V(r)
$$

where $\vec{A}$ is the vector field of the photons. Here we can use the Coulomb gauge for which $\vec{p} \cdot \vec{A}=\frac{\hbar}{i} \vec{\nabla} \cdot \vec{A}=0 \rightarrow p \cdot A+A \cdot p=2 p \cdot A$. The Hamiltonian then becomes

$$
H \approx \frac{p^{2}}{2 m}+\frac{e}{m c} \vec{A} \cdot \vec{p}+V(r)=H^{\circ}+H^{1}
$$




\subsection{Introduction}

where $H^{\circ}=\frac{p^{2}}{2 m}+V(r)$ is the Hamiltonian of the system without the incident light, and $H^{1}=\frac{e}{m c} \vec{A} \cdot \vec{p}$ is the perturbing potential.

On the other hand, the decay rate of an initial state $\psi_{i}$ into some particular final state $\psi_{f}$ is given by Fermi's golden rule (derived from timedependant perturbation theory) as:

$$
R_{i \rightarrow f}=\sum_{i \rightarrow f} \frac{2 \pi}{\hbar}\left|\left\langle\psi_{f}\left|H^{1}\right| \psi_{i}\right\rangle\right|^{2} \delta\left(E_{f}-E_{i}-\hbar \omega\right) .
$$

$A$ is the radiation field operator which can be described using the following equations:

$$
A=A_{\circ} \hat{\epsilon}\left[e^{i(\vec{k} \cdot \vec{r}-\omega t)}+e^{-i(\vec{k} \cdot \vec{r}-\omega t)}\right]
$$

where $\hat{\epsilon}$ gives the direction (or polarization) of the vector potential. On the other hand, energy inside a volume $V$ is:

$$
\operatorname{Energy}_{V}=N \hbar \omega=\frac{\omega^{2}}{2 \pi c^{2}} V\left|A_{\circ}\right|^{2},
$$

Therefore,

$$
\rightarrow\left|\left\langle\psi_{f}\left|H^{1}\right| \psi_{i}\right\rangle\right|=\left|\left\langle f\left|\frac{e}{m c} \vec{A} \cdot \vec{p}\right| i\right\rangle\right|=\frac{e}{m c}\left(\frac{2 \pi^{2}}{\omega V}\right)^{\frac{1}{2}}\left|\left\langle\psi_{f}\left|e^{-i \vec{k} \cdot \vec{r}} \hat{\epsilon} \cdot \vec{p}\right| \psi_{i}\right\rangle\right|
$$

For soft x-ray absorption spectra (i.e $\hbar \omega<1 \mathrm{KeV}$ ), the wavelength is much larger than the radial extend of the core level wave functions so we can make the following approximation which is called the electric dipole approximation:

$$
e^{-i \vec{k} \cdot \vec{r}} \approx 1-i \vec{k} \cdot \vec{r}+\ldots \approx 1
$$

Furthermore, if $H=\frac{p^{2}}{2 m}+V(\vec{r})$, then $[V(\vec{r}), \vec{r}]=0$ and

$$
\begin{aligned}
& {[H, \vec{r}]=\frac{\hbar}{i} \frac{p}{m} \rightarrow \vec{p}=\frac{i m}{\hbar}[H, \vec{r}]} \\
& \rightarrow\left\langle\psi_{f}\left|e^{-i \vec{k} \cdot \vec{r}} \hat{\epsilon} \cdot \vec{p}\right| \psi_{i}\right\rangle \simeq \hat{\epsilon} \cdot\left\langle\psi_{f}|\vec{p}| \psi_{i}\right\rangle \\
& =\frac{i m \hat{\epsilon}}{\hbar} \cdot\left\langle\psi_{f}|[H, \vec{r}]| \psi_{i}\right\rangle=\frac{i m\left(E_{f}-E_{i}\right)}{\hbar} \cdot\left\langle\psi_{f}|\hat{\epsilon} \cdot \vec{r}| \psi_{i}\right\rangle
\end{aligned}
$$

From Eq. 3.3 and 3.8 the absorption rate of dipole excitations is found to be: 


\subsection{Introduction}

$$
R_{i \rightarrow f} \propto \sum_{i \rightarrow f} \frac{2 \pi}{\hbar}\left|\left\langle\psi_{f}|\hat{\epsilon} . \vec{r}| \psi_{i}\right\rangle\right|^{2} \delta\left(E_{f}-E_{i}-\hbar \omega\right)
$$

Then, we can write the $\hat{\epsilon} . \hat{r}$ term in terms of spherical harmonics:

$$
\begin{gathered}
\hat{\epsilon} . \hat{r}=\epsilon_{x} \sin \phi+\epsilon_{y} \sin \phi+\epsilon_{z} \cos \theta \\
=\sqrt{\frac{4 \pi}{3}}\left[\epsilon_{z} Y_{10}-\frac{\left(\epsilon_{x}+i \epsilon_{y}\right)}{\sqrt{2}} Y_{11}+\frac{\left(\epsilon_{x}+i \epsilon_{y}\right)}{\sqrt{2}} Y_{l-1}\right] \\
\left\langle\psi_{f}|\hat{\epsilon} \cdot \vec{r}| \psi_{i}\right\rangle=\sqrt{\frac{4 \pi}{3}} \int_{0}^{\infty} r^{3} d r R_{n_{f} l_{f}}^{\star} R_{n_{i} l_{i}} \\
\int d \Omega Y_{l_{f} m_{f}}^{\star}\left(\epsilon_{z} Y_{10}+\frac{-\epsilon_{x}+i \epsilon_{y}}{\sqrt{2}} Y_{11}+\frac{\epsilon_{x}+i \epsilon_{y}}{\sqrt{2}} Y_{1-1}\right) Y_{l_{i} m_{i}}
\end{gathered}
$$

After some additional calculational steps using the Clebsch-Gordan coefficients, the final result for the matrix elements is found to be:

$$
\begin{aligned}
& \left\langle\psi_{f}|\hat{\epsilon} . \vec{r}| \psi_{i}\right\rangle=\sqrt{\frac{2\left(l_{i}+1\right)}{2\left(l_{f}+1\right)}}\left\langle l_{f} 0 \mid l_{i} 100\right\rangle \int_{0}^{\infty} r^{3} d r R_{n_{f} l_{f}}^{\star} R_{n_{i} l_{i}} \\
& \left(\epsilon_{z}\left\langle l_{f} m_{f} \mid l_{i} 1 m_{i} 0\right\rangle+\frac{-\epsilon_{x}+i \epsilon_{y}}{\sqrt{2}}\left\langle l_{f} m_{f} \mid l_{i} 1 m_{i} 1\right\rangle+\frac{\epsilon_{x}+i \epsilon_{y}}{\sqrt{2}}\left\langle l_{f} m_{f} \mid l_{i} 1 m_{i}-1\right\rangle\right)
\end{aligned}
$$

The above Clebsch-Gordan coefficients will be non-zero only if $\Delta \ell=$ $0, \pm 1$. On the other hand, since $Y_{1 m}$ terms are odd parity, if the other two spherical harmonics are the same the integral will be zero; thus, $\Delta \ell \neq 0$ otherwise the transition is forbidden. Consequently, the $z$ components of the angular momentum, $m_{l}$, have to be either 0 or $\pm 1: \Delta m_{l}=0, \pm 1$. Note, there are no operators to change the spin, therefore $\Delta s=0$. These restrictions are called Dipole Selection Rules.

The more general dipole selection rules for multi-electron atoms are shown below with uppercase symbols. It also shows the restrictions on the total angular momentum number $J=L+S$, and its projection along the $z$ axis , $M_{J}$. These quantum numbers are important mainly if the spin-orbit coupling is of importance in which case the spin and orbital parts cannot be treated separately. Because when the spin-orbit interaction term (L.S) is included in the Hamiltonian, the spin $(\mathrm{S})$ and orbit $(\mathrm{L})$ operators do not commute with the Hamiltonian anymore. 


$$
\begin{aligned}
& \Delta L=0, \pm 1, L=0 \not \rightarrow L=0 \\
& \Delta M_{J}=0, \pm 1 \\
& \Delta J=0, \pm 1 \\
& \Delta S=0
\end{aligned}
$$

Note that the above selection rules are valid only for the electric dipole approximation which is valid in the soft x-ray absorption process. There is also one absolute selection rule that comes from angular momentum conservation. Since a photon has a spin of 1 , no $j=0 \rightarrow j=0$ transition is allowed in any order of approximation [12, 72, 73. These selections rules play an important role in the theory of core-level x-ray spectroscopy techniques.

In perovskite compounds, the K edge XAS of the ligands is of particular interest where a lot can be learned about the electronic structure of the material especially the role of ligands. Having the above dipole selection rules of $\Delta \ell= \pm 1$, implies that if we are exciting a core $1 s$ electron with $l=0$ (K-edge excitation), the electron can only go to unoccupied states of $p$ character $(l=1)$. Thus, the $K$ edge NEXAFS spectra provide a good approximation to the ligand $2 p$ unoccupied Density Of States (DOS) [1].

The intensity of the XAS spectra can, in principle, be given by the Eq. 3.9. however, this equation is applicable when the final state has an infinite lifetime. For a finite lifetime in the final state, the $\delta$ function has to be replaced by a Lorentzian profile as:

$$
I \propto R_{f i} \propto \sum_{i \rightarrow f}\left|\left\langle\psi_{f}|\hat{\epsilon} . \vec{r}| \psi_{i}\right\rangle\right|^{2} \frac{\Gamma_{f}}{\left(E_{i}-E_{f}+\omega\right)^{2}+\Gamma_{f}^{2}},
$$

where $\Gamma_{f}$ is the Full Width at Half Maximum (FWHM) of the final state's lifetime-induced Lorentzian broadening [11, 12, 74, 75].

On the other hand, XES is a second order process meaning that after a core electron is excited by incident x-rays, an electron decays from an occupied valence band into the empty core state emitting a photon, which is being detected. In a resonant x-ray emission, the incident x-ray energy is tuned to resonate with the excitation edge of the core electron. Therefore, the intermediate state in the RXES is the same as the final state in the XAS. The schematic representation of these core-level excitations is shown in Fig. 3.2 .

Specifically, oxygen K-edge RXES happens when the incident x-ray energy is the same as the energy to excite an oxygen $1 s$ core electron to an unoccupied $p$ state where then an electron decays from an occupied $p$-like 


\subsection{Introduction}

state into the empty core $s$ emitting a photon (due to the dipole selection rule). Thus, the oxygen K-edge XAS and RXES techniques make a powerful complementary tool for probing the unoccupied and occupied $p$ partial density of states.

However, one must remember XAS+RXES does not precisely probe the oxygen- $p$ DOS. That is because through these measurements a core electron is removed and a core hole is created. Thus, these techniques, in fact, probe the states in the presence of a core hole potential. Thankfully, it is found that for many materials this core hole potential does not affect the DOS strongly if it is small compared to the bandwidth of the valence states and we can still use these techniques for probing the DOS reliably[76].

As discussed above, XES is a second order process where the probability of emission of photons of energy $\hbar \omega_{k}^{\prime}$ in the solid angle $d \Omega_{k^{\prime}}$ is given by the Kramers-Heisenberg formula for second order processes[74, 77]:

$$
\frac{d^{2} \sigma}{d \Omega_{k^{\prime}} d\left(\hbar \omega_{k}^{\prime}\right)}=\frac{\omega_{k}^{\prime}}{\omega_{k}} \sum_{|f\rangle}\left|\sum_{|n\rangle} \frac{\left\langle f\left|T^{\dagger}\right| n\right\rangle\langle n|T| i\rangle}{E_{i}-E_{n}+\hbar \omega_{k}+i \frac{\Gamma_{n}}{2}}\right|^{2} \delta\left(E_{i}-E_{f}+\hbar \omega_{k}-\hbar \omega_{k}^{\prime}\right)
$$

where $E_{i}, E_{n}, E_{f}$ are the initial, intermediate and final states of the system through the process, respectively. $\hbar \omega_{k}$ is the energy of the incident photon, and $\Gamma_{n}$ is the intrinsic broadening of the intermediate state.

\subsubsection{Detection Techniques}

The x-ray absorption rate or the attenuation of light is related to the sample thickness and its properties via the Beer-Lambert law:

$$
I(\omega)=I_{0}(\omega) e^{\mu(\omega) D} \Rightarrow \mu(\omega)=-\frac{1}{D} \ln \frac{I(\omega)}{I_{0}(\omega)}
$$

where $I$ and $I_{0}$ are the transmitted and incident beam intensities respectively. $\omega$ is the energy of the x-rays. $D$ is the sample thickness, and $\mu$ is called the "total linear absorption coefficient" or the "attenuation coefficient". This coefficient is related to the absorption cross-section and depends on the material's properties [71]. Experimentally, one can obtain the absorption cross section by measuring the incident and transmitted light through the sample namely in the transmission mode.

However, there can be a serious issue with the above transmission probing technique. First, it requires an energy-dependent optimization of thickness and density for each sample. Second, if $\mu(\omega) D>>1$ photons cannot 


\subsection{Introduction}

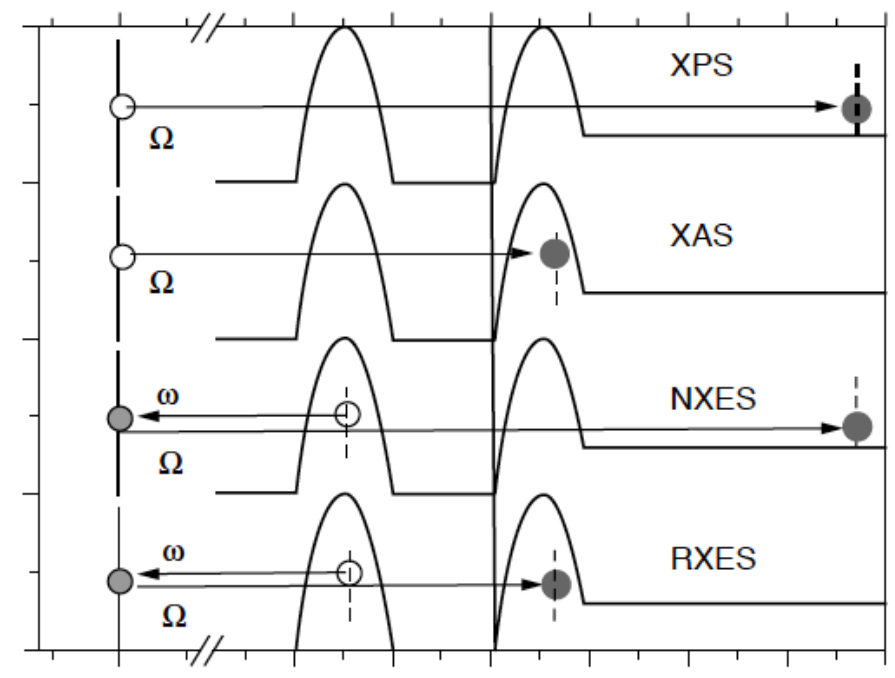

Figure 3.2: The schematic representation of x-ray photoemission/absorption, normal and resonant emission processes. [12]

pass through the sample then one will detect no signal. Even if the attenuation is not big enough to stop the incident photons at all energies, it can still cause artificial suppression of the absorption peaks and affect the analysis [11]. An alternative indirect approach to avoid this issue uses the yields techniques.

In XAS, after the incident photons are absorbed, the sample is left in an excited state which quickly decays by emitting electrons or photons. It is often found in practice that the number of transmitted photons (absorption signal) is proportional to the number of these emitted photons or electrons. If one detects these emitted electrons as a function of incident photon energy, the technique is called Total Electron Yield or TEY. Since the mean free path of the electrons is very short, TEY is quite surface sensitive with a probing depth of several $n m[71]$. On the other hand, if one records and detects the emitted photons as a way to measure XAS, it is called Total Fluorescence Yield ( TFY) technique. TFY has a larger probing depth than TEY because the soft X-ray photons have larger penetration depths of about $\approx 100 \mathrm{~nm}[11$. These techniques are depicted in Fig. 3.3 schematically. 


\subsection{Introduction}

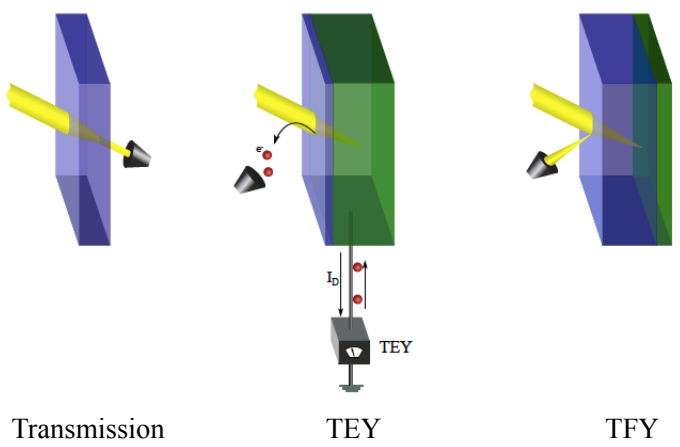

Figure 3.3: Some standard XAS detection techniques. The blue regions qualitatively represent the detection depths of each technique. [11]

\subsubsection{REIXS Beam-line at CLS}

Our XAS and RXES experiments were carried out at the Resonant Elastic and Inelastic X-ray Spectroscopy (REIXS) beam-line at the Canadian Light Source (CLS), a third-generation synchrotron facility. The REIXS beam-line is a cutting-edge soft x-ray scattering facility located at the 10ID-2 straight section, Canadian Light Source, Saskatoon, Saskatchewan, Canada[13].

Generally, in a synchrotron, the magnets are placed at regular positions on the circumference of a circle. Relativistic electrons moving in the horizontal plane are accelerated radially when they pass through the vertically oriented magnetic fields. By strategic placements of the magnets, the electrons can be kept in a stable circulating orbit consisting of straight sections interrupted by the bending magnets. If charged particles are accelerated they emit electromagnetic radiation which is in the x-ray range for strongly relativistic electrons travelling in strong enough magnetic fields. So x-rays are emitted at each of the positions of the bending magnets which then can be used after some focusing and monochromatization [78. The radiation has a substantial energy spread, so x-ray monochromators are used to select the energy which can be scanned through the energy range of interest. In the higher generation facilities, the above mentioned straight sections are replaced by a periodic array of permanent magnets with alternating up and 


\subsection{Introduction}

down magnetic fields causing an oscillating motion of the electrons and much more intense and directed $\mathrm{x}$ radiation.

The CLS beam-line, as a third generation synchrotron, allows for various photon-in and photon-out experiments with full polarization control, high flux and high resolution and at different temperatures. At the REIXS beamline station the following techniques are allowed:

- X-ray Absorption Spectroscopy (XAS):

- Total electron yield (TEY)

- Total fluorescence yield (TFY)

- Resonant Inelastic X-ray Scattering (RIXS)

- Resonant X-Ray Scattering (RXS)

- X-ray Absorption Fine Structure (XAFS)

- X-ray Emission Spectroscopy (XES)

- X-ray Magnetic Linear Dichroism (XMLD)

- Surface Science Facility End-station:

- Molecular Beam Epitaxy (MBE) with RHEED/LEED

- X-ray Photoelectron Spectroscopy (XPS)

$-\ldots$

At this end-station, the Variable-Line-Spacing Plane Grating Monochromator (VLS-PGM) is used to optimize the beam flux and to select the photon energy. VLS-PGM has a combination of different mirror coatings $(\mathrm{Ni}$, $\mathrm{Si}, \mathrm{C}$, and $\mathrm{Au}$ ) and gratings to help to suppress the higher order harmonics. Some other specifications of the incident beam such as the flux, energy range, spot size, etc. are presented in table. 3.5. Our XPS measurements in chapter 4 were also performed in the Surface Science Facility end-station of the same beam-line. 


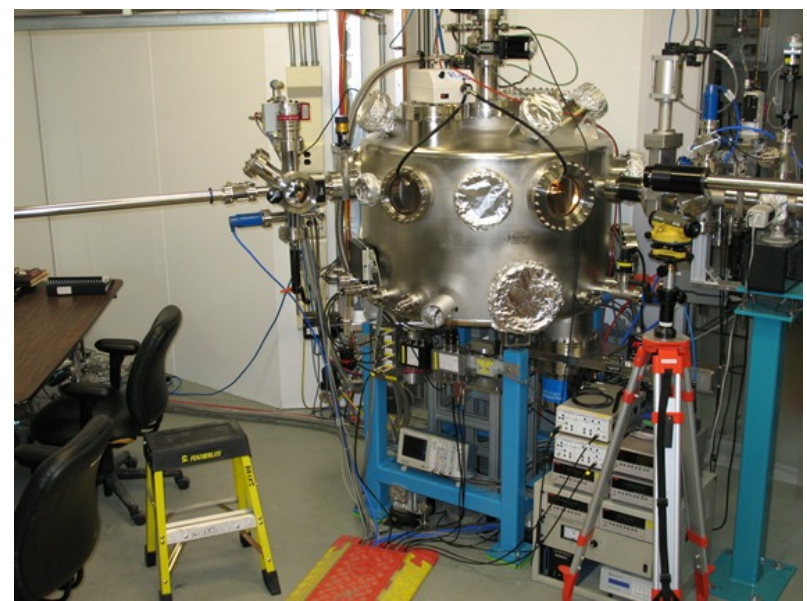

Figure 3.4: XAS machine at the REIXS end-station at CLS [13].

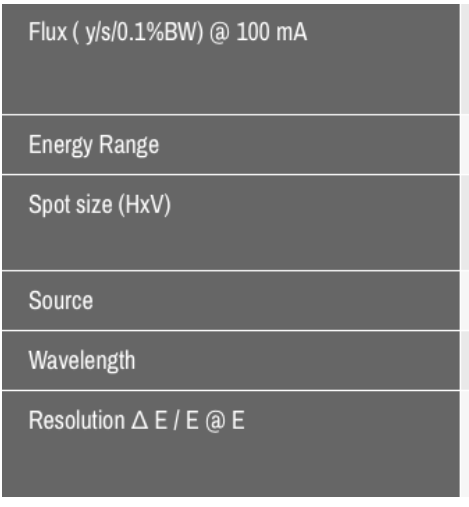
$1 \times 10^{12} @ 100 \mathrm{eV}$
$5 \times 10^{11} @ 1000 \mathrm{eV}$
$80-2000 \mathrm{eV}$
RSXS: $250 \times 150 \mu \mathrm{m}$
XES: $60 \times 10 \mu \mathrm{m}$
Elliptically Polarizing Undulator (EPU)
$155-6.2 \AA$
$5 \times 10^{-5} @ 100 \mathrm{eV}$
$1.3 \times 10^{-4} @ 1000 \mathrm{eV}$

Figure 3.5: Some specifications of the REIXS beam-line end-station at CLS [14].

\subsection{Oxygen K-edge XAS Results}

The oxygen K-edge XAS measurements were carried out on a single crystal of $\mathrm{BaBiO}_{3}$ at temperatures between $20 \mathrm{~K}$ and $400 \mathrm{~K}$ in $15 \mathrm{~K}$ intervals and with polarizations perpendicular and parallel to the sample surface plane. As shown in Fig. 3.6, no significant change in the overall structure of the spectrum is found with changing temperature or the polarization except for 


\subsection{Oxygen K-edge XAS Results}

an expected slight broadening at the higher temperatures. The spectra are normalized with respect to the pre-edge and above-edge intensities (at 520 and $570 \mathrm{eV}$, respectively). The most significant feature of the spectrum is the sharp pre-peak at about $529 \mathrm{eV}$. It suggests a high oxygen $p$ hole density in the ground state. The structure of the spectrum will be discussed more in the next sections when it is compared to the unoccupied oxygen $p$ projected DOS from DFT (LDA).

During the experiment, both bulk sensitive TFY and the more surface sensitive TEY signals were recorded simultaneously. However, as shown in Fig. 3.7 and was also reported earlier [79], the reproducibility of TFY signal is better than that of TEY. This is most probably due to the sample's surface contamination and TFY known lower surface sensitivity. Therefore, the TFY signals are presented and studied in this work. 


\subsection{Oxygen K-edge XAS Results}
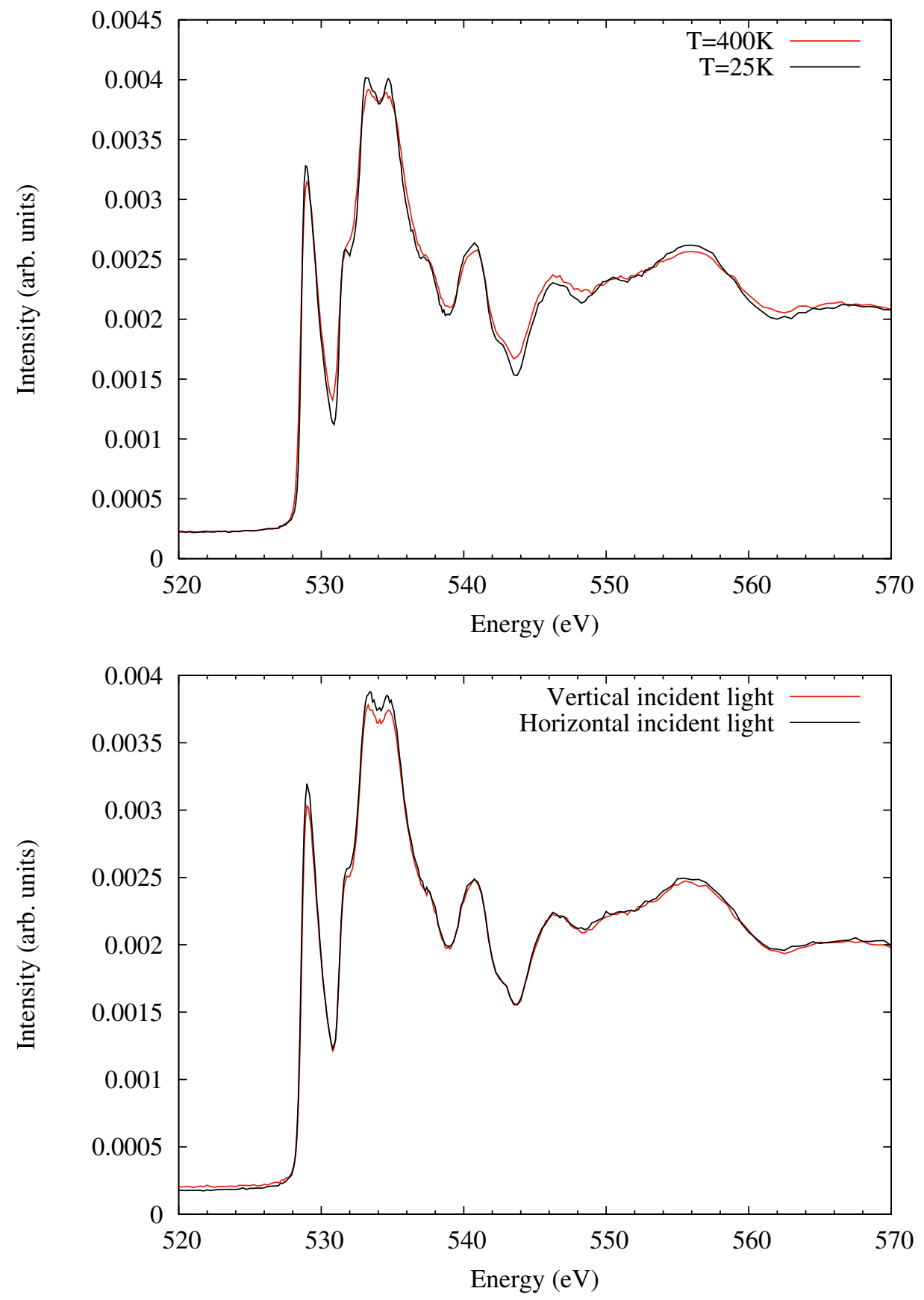

Figure 3.6: Oxygen K-edge XAS of single-crystal $\mathrm{BaBiO}_{3}$ top: measured at $20 \mathrm{~K}$ and $400 \mathrm{~K}$ with vertical polarization. bottom: with polarizations perpendicular (vertical) and parallel (horizontal) to the sample surface plane at room temperature. No significant temperature or polarization dependene is observed. 


\subsection{Oxygen K-edge XAS Results}
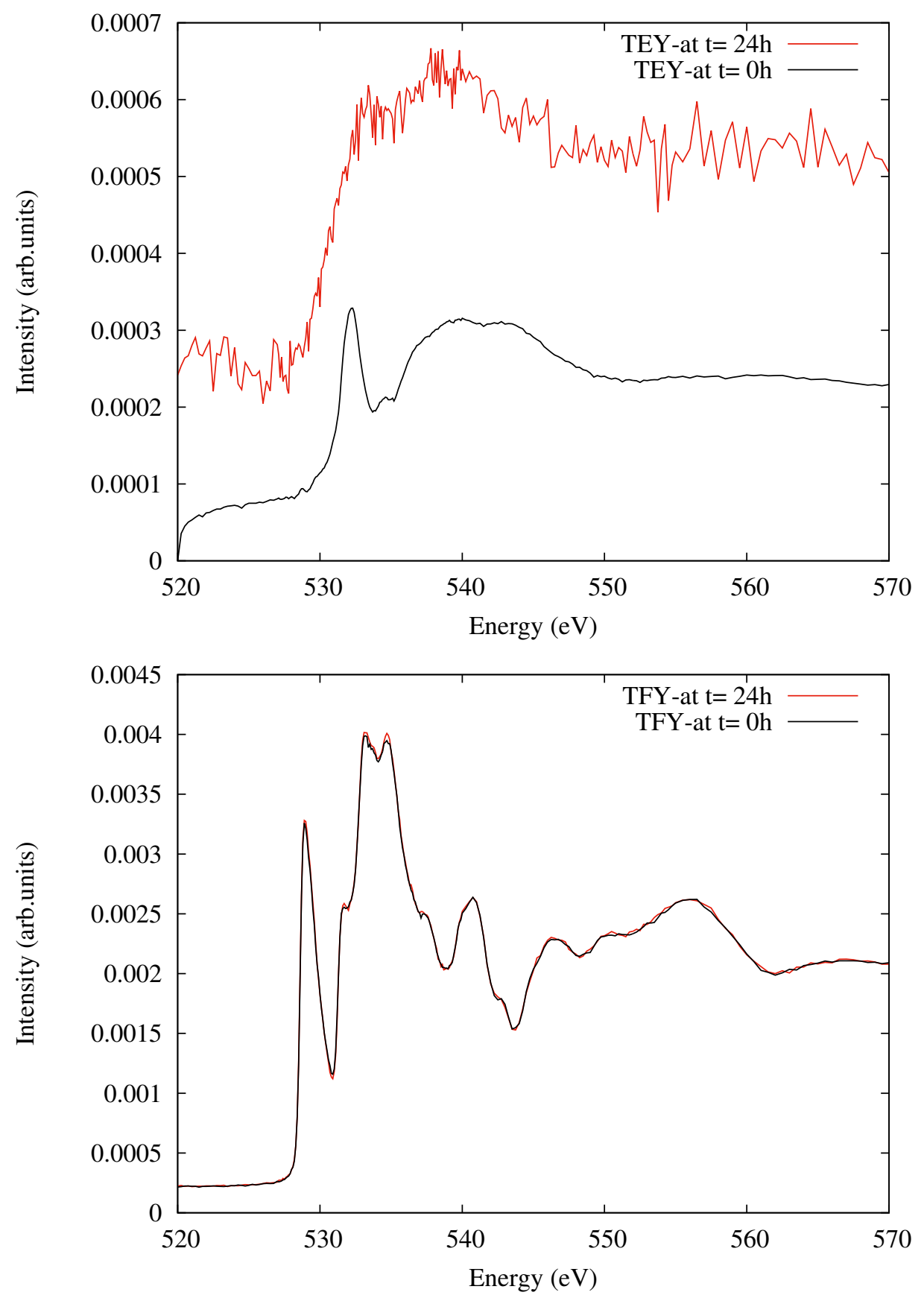

Figure 3.7: The reproducibility of the top: TEY and bottom: TFY signals are tested for the oxygen K-edge XAS of single-crystal $\mathrm{BaBiO}_{3}$. The measurements are repeated after 24 hours while sample was kept in vacuum. The TFY is found reproducible over time unlike the TEY. 


\subsection{Oxygen K-edge RXES Results}

The oxygen K-edge resonant x-ray emission measurements were performed at different resonant $\mathrm{x}$-ray incident energies taken from the corresponding XAS spectrum. The result is presented in Fig. 3.8. The peak at the highest energy is called the "elastic line" or the "zero-loss" peak. Through this RXES process, a core $1 s$ electron is excited to a conduction band, and then another valence electron decays back emitting a photon. However, if the same excited electron decays back, it gives rise to a peak at the same incident energy, namely the "zero-loss" peak. These peaks usually are not rich with information and only used to calibrate the energy. The more significant features observed in the spectra are; a peak at about $527 \mathrm{eV}$, a strong asymmetric peak about $2 \mathrm{eV}$ away (at $525 \mathrm{eV}$ ), and another bump at about $9 \mathrm{eV}$ further down (at $518 \mathrm{eV}$ ). The spectrum will be studied further in the next section when it is compared to the occupied oxygen $p$ projected density of states from DFT (LDA).

No significant change is observed in the overall shape of the spectrum for the three different resonant energies except for a slight broadening and shift towards the elastic line for the higher incident energies. Observing some differences is not unexpected as with having different resonant incident energies, the excited electron occupies the conduction band at a different energy. Thus, its potential can affect the final transitions differently. In fact, studying this effect in detail for a few different resonant energies can potentially give useful information about the material's conduction band; however, it is not part of this work. 


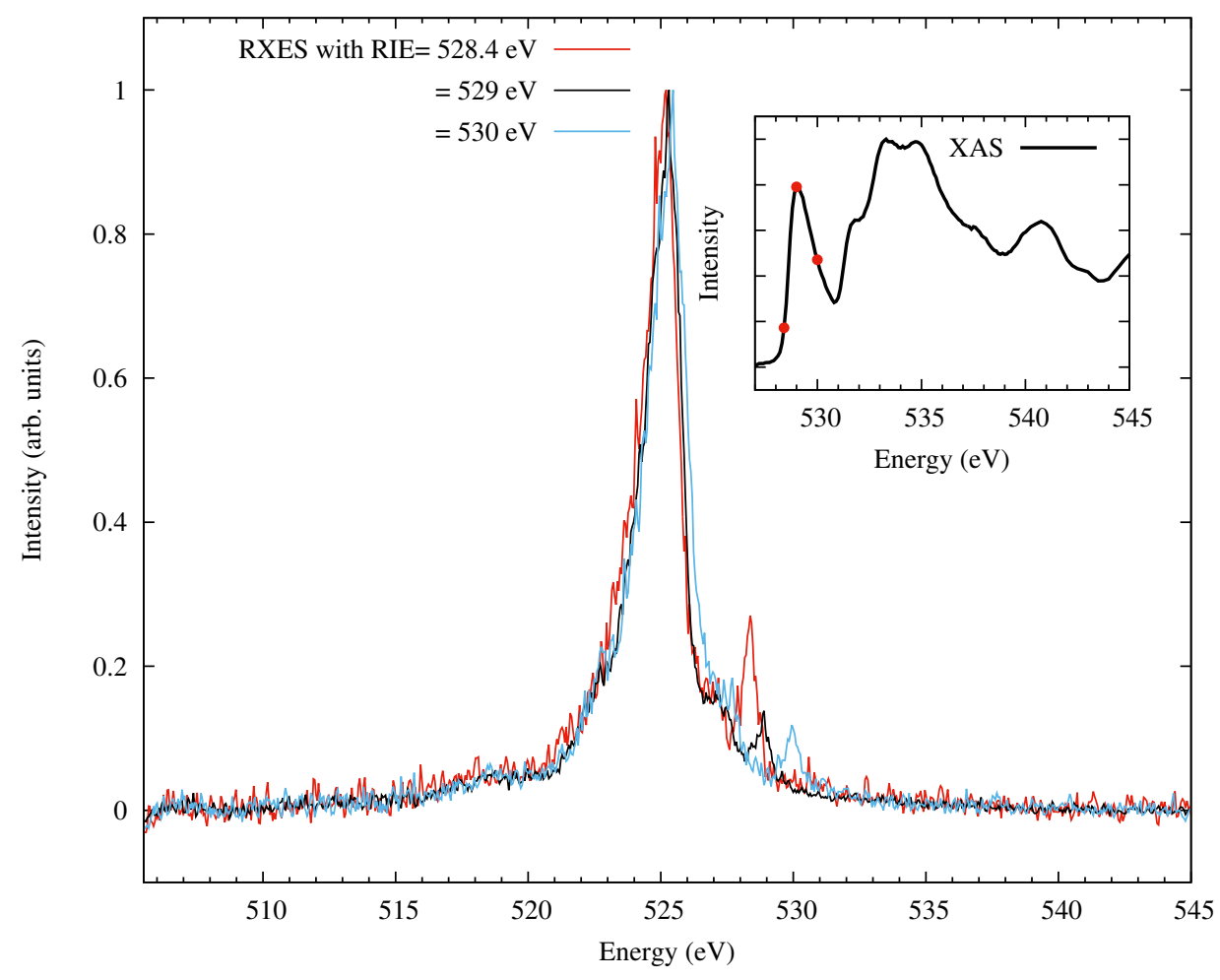

Figure 3.8: Main: oxygen K-edge resonant x-ray emission spectra on a single crystal of $\mathrm{BaBiO}_{3}$ at three different Resonant Incident Energies (RIE). Inset: oxygen K-edge XAS spectrum. The red dots show the resonant incident energies used for the RXES measurement.

\subsection{Theoretical Interpretation of The Experiment}

Density Functional Theory (DFT) in the Local Density Approximation (LDA) is one of the most powerful and versatile methods in condensed matter physics. In DFT, the wave-function of a many-electron system is assumed to be described by the Slater determinant of delocalized one-electron Bloch states. Thus, it is in fact a single-particle theory that accounts for correlations only in the form of effective single-particle potentials (a mean-fieldtype approach) [80]. This assumption can be a serious issue for strongly correlated systems. For example, when DFT predicts the antiferromagnetic 
insulator $\mathrm{La}_{2} \mathrm{CuO}_{4}$ to be a non-magnetic metal [81]. However, it is quite successful for weakly correlated systems i.e. the systems in which the bandwidths are much wider than the Coulomb interactions $(W>>U)$ where it is reasonable to neglect the electron-electron correlations. In such cases, it obtains the correct ground state and magnetic structure and accurate crystal structure for a wide range of materials. We use results from DFT to study our experimental results.

As mentioned earlier, we used XAS+RXES to probe the $\mathrm{BaBiO}_{3} p$ like density of states and investigate the electronic structure of the ligand oxygens. We compare the results with the oxygen- $p$ projected DOS from DFT(LDA). These calculations were done in our group by Kateryna Foyevtsova et al. The computational settings for these calculations are the same as their previously published report [4] on the sister compound $\mathrm{SrBiO}_{3}$ but using the $\mathrm{BaBiO}_{3}$ structure. This band structure calculation suggests that: "the breathing distortion is accompanied by spatial condensation of hole pairs into local, molecular-like orbitals of the $A_{1 g}$ symmetry composed of $\mathrm{O}-2 p \sigma$ and $\mathrm{Bi}-6 s$ atomic orbitals of collapsed $\mathrm{BiO}_{6}$ octahedra. The primary importance of oxygen $p$ states is thus revealed, in contrast to a popular picture of a purely ionic $\mathrm{Bi}^{3+} / \mathrm{Bi}^{5+}$ charge disproportionation. Octahedra tilting is shown to enhance the breathing instability by means of a nonuniform band narrowing. [4]"

The comparison is presented in Fig. 3.9 where the calculated projected density of states is convoluted with a Lorentzian with a Full Width at Half Maximum of $0.7 \mathrm{eV}$ for comparison to the experiment. The energy axis for the experiment has also been shifted by $-528.5 \mathrm{eV}$. As shown, we get a reasonably good agreement between the experiment and the theory in terms of the energy position of the bands and their relative intensities. RXES even gives the deep $\mathrm{Bi} 6 s$ states at about $-9 \mathrm{eV}$ predicted by the DFT due to the hybridization between the oxygens and the bismuth states.

The measured sharp XAS pre-peak at $0.5 \mathrm{eV}$ (or $529 \mathrm{eV}$ for the XAS incident beam energy) gives evidence for a high oxygen $p$ hole density in the $\mathrm{BaBiO}_{3}$ ground state. This, along with the general agreement between the DFT and the experiment, supports the proposed bond disproportionation picture in which the holes mainly reside on the oxygen anions rather the $\mathrm{Bi}$ $6 s$ cations as opposed to the conventional purely ionic charge disproportionation description. 


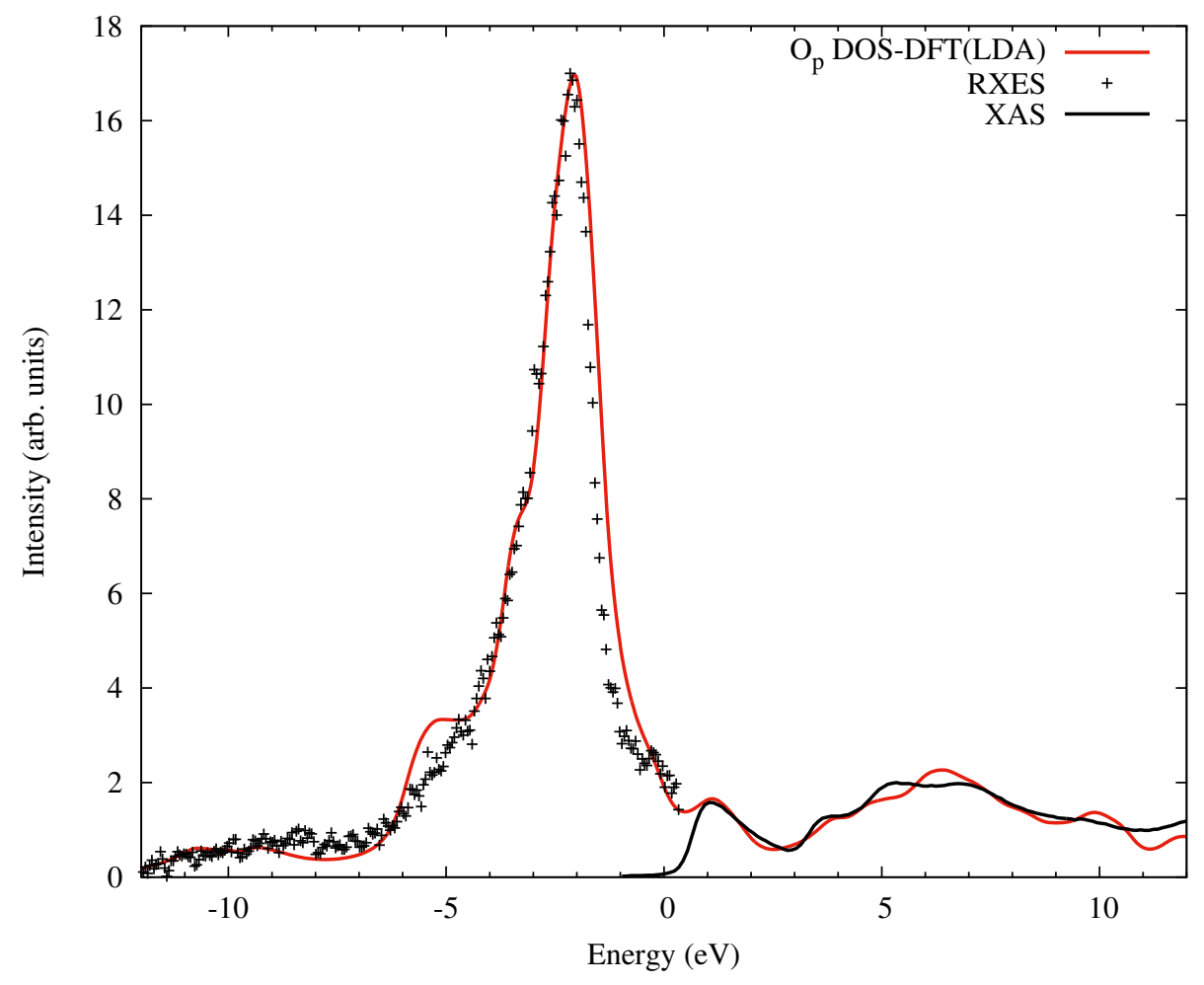

Figure 3.9: Oxygen K-edge RXES and XAS spectra measured on a $\mathrm{BaBiO}_{3}$ single crystal and the DFT(LDA) oxygen $p$ projected density of states of $\mathrm{BaBiO}_{3}$. The energy has been shifted by $-528.5 \mathrm{eV}$ for the experimental axis to facilitate comparison with the calculation.

Nevertheless, some disagreements can still be observed in this comparison mainly at $5 \mathrm{eV}$ and $7 \mathrm{eV}$. We also get lower intensity at higher energies than expected by DFT as also is shown in Fig. 3.10. This disagreement can stem from the fact that, as mentioned in previous section, through XAS+RXES we do not exactly probe the density of states of a charge neutral system as is assumed by DFT. Through these processes, we excite a core electron and create a core hole potential. Therefore, what is measured is the DOS in the presence of such potential. Moreover, on the other hand, DFT as a mean-field single particle theory cannot treat such local potentials properly. Mostly for educational purposes, we go on and compare our experimental result to some commonly used "modifications" to DOS from 
DFT.

Previously in Fig. 3.9, when the oxygen K-edge XAS+RXES experimental result was compared to the oxygen- $p$ projected DOS from DFT(LDA), it was assumed that the hole created through the spectroscopy process has no effect on the final states and, therefore, on the spectrum. As shown before, this assumption works reasonably well for this material but is not perfect especially for XAS where a core-hole exists in the final state. Through the $\mathrm{x}$-ray emission, the hole exists in the valence state and therefore can be well screened and have less effect on the final state. The experimental resolution is also worse for the XES so not all of the details can be observed, regardless.

In this section, we compare and discuss a few other computational approaches that are commonly used to simulate the XAS+RXES results. For the first modification, in order to improve the accuracy of the calculation, the XAS spectrum is calculated based on the Fermi's Golden rule, as was described previously, using the corresponding transition matrix between the initial state and the selection rule allowed final states. This calculation is done using the XSPEC package in the Wien2K program. The result has been compared to the experiment in Fig. 3.10 middle panel. As shown, this modification does not change the spectrum much compared to the previous version where it was compared to the oxygen- $p$ projected DOS. However, it helps at the higher energies by giving a lower intensity compared to the previous version and therefore closer to the experiment. That is perhaps due to the fact that here the transition probability from a core $1 s$ to $p$ orbitals is calculated and this probability is less for transitions to higher energies compared to the states right above the Fermi which was not taken into account previously.

Another commonly used approach to "improve" the DFT DOS correspondence to XAS is including the core-hole in the calculation as follows: A supercell is constructed and then one core electron is removed from one of the atoms in the supercell and one electron is added to the conduction band as a uniform background charge to treat the core-hole potential effect on the XAS result. The result of this approach for a $2 \times 2 \times 2$ supercell is shown in Fig. 3.10 bottom panel.

As shown, not only does this step not improve but even worsens the result. This approach is frequently used by the DFT community to find the core level spectroscopy result, but this example demonstrates the fundamental problem with this solution. When in DFT the additional electron is added to the conduction band it means these unoccupied states are now less unoccupied. Therefore, when the transition happens from the core state, there is less hole density at the unoccupied band, and therefore the XAS 


\subsection{Theoretical Interpretation of The Experiment}

pre-peak loses its intensity compared to the real scenario. Thus, this "approximation" treats the oxygen more like fluorine which is not correct. 


\subsection{Theoretical Interpretation of The Experiment}
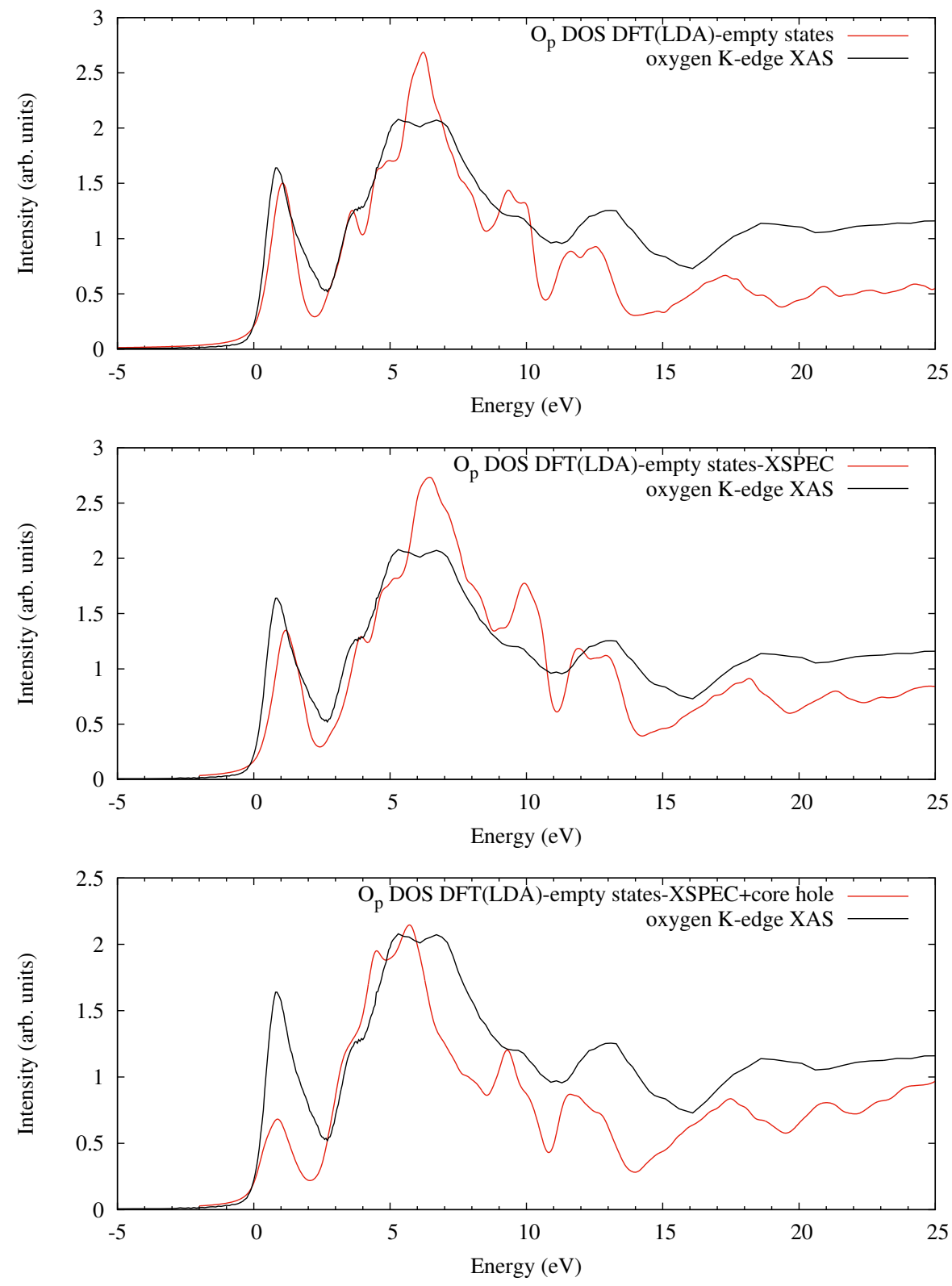

Figure 3.10: Oxygen K-edge XAS compared to oxygen $p$ projected density of unoccupied states from top: DFT(LDA). middle: DFT(LDA) and the XSPEC package which takes into account the transition probabilities in calculating the intensity. bottom: DFT (LDA) on a $2 \times 2 \times 2$ supercell with a core-hole. 


\subsection{Conclusion}

\subsection{Conclusion}

We used oxygen K-edge XAS and RXES on the $\mathrm{BaBiO}_{3}$ single crystal to study the oxygens' bonding and anti bonding $p$-bands close to the chemical potential. The strong pre-peak found at the XAS suggests a high hole concentration in the material. The XAS+RXES result was compared to the DFT(LDA) oxygen- $p$ projected density of states and a reasonable agreement was found. The presence of the mentioned XAS pre-peak and this achieved agreement give evidence for the $\mathrm{BaBiO}_{3}$ bond- disproportionation insulating mechanism where holes mainly reside on the ligand oxygens rather than the purely ionic charge- disproportionation picture.

We also showed that adding a core-hole potential to the DFT calculations to simulate the XAS process is not an appropriate approach. It gives a much lower intensity in the XAS pre-peak because adding an electron (to screen the core-hole) makes the unoccupied band less empty and lowers its true hole density. 


\section{Chapter 4}

\section{X-ray Photoemission}

In this chapter, we proceed with the investigation of the electronic structure of the $\mathrm{BaBiO}_{3}$ using oxygen $1 s$ x-ray photoemission technique. The measurement is done on a home-grown $\mathrm{BaBiO}_{3}$ single crystal described in chapter 2. The sample is cleaned systematically in order to achieve a reliable oxygen $1 s$ spectrum. The experiments are performed at Canadian Light Source Surface Science laboratory.

\subsection{Introduction}

X-ray photoemission spectroscopy (XPS) is one of the most widely used techniques in studying the composition and the electronic structure of materials. XPS quantification and its fundamental physics can be traced back to Einstein's photoelectric theory in 1905 [82. However, its main development did not happen until the 1950s when Kai Siegbahn and his research group at the University of Uppsala, Sweden contributed significantly to its development and improvement as a detection technique. In 1981, Siegbahn received a Nobel Prize for his contribution in developing the high-resolution XPS, which was mostly known as Electron Spectroscopy for Chemical Analysis (ESCA) at the time [83].

The XPS process involves ejecting core electrons from the material by incident x-ray photons. The emitted electrons are then detected, and their kinetic energy is measured. However, this kinetic energy depends on the energy of the incident x-rays and therefore is not intrinsic to the material. On the other hand, the binding energy of the electron is a characteristic property of the excited element. Thankfully, these two energies are related to each other with:

$$
E_{b}=E_{h \nu}-E_{k}-W,
$$

where $E_{b}$ is the binding energy of the emitted electron, $E_{h \nu}$ is the incident photon energy, $E_{k}$ is the measured kinetic energy of the emitted electron, and $W$ is the work function of the material if the binding energy is referenced 


\subsection{Introduction}

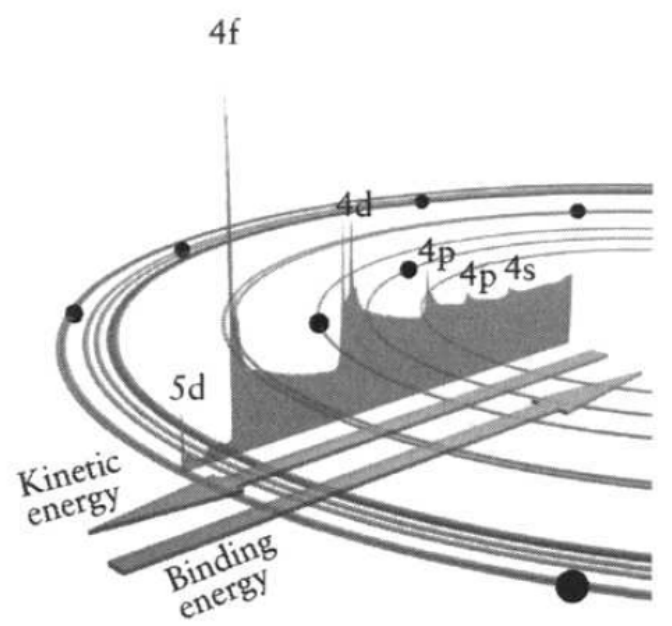

Figure 4.1: XPS spectrum of lead illustrating schematically how exciting electrons from each orbital can give rise to a distinct peak in the spectrum [3]. Those electrons that are excited and leave the surface without an energy loss produce the characteristic peaks and those that undergo inelastic scattering and energy loss processes contribute to the background.

to the Fermi energy. All the three quantities on the right hand side of the Eq. 4.1 are either known or can be measured. Therefore, the electron's binding energy can be found straightforwardly.

The XPS spectrum is then obtained by plotting the number of ejected electrons per energy interval versus their binding energy. It consists of a series of distinct lines or "peaks" corresponding to the sequence of the occupied core levels of the material. An example of such spectrum is shown in Fig. 4.1. These binding energies are characteristic of each element, and therefore XPS spectra can serve to detect the chemical elements of the system under study.

The schematic diagram of the core $1 s$ XPS process is shown in Fig. 4.2 where an electron is ejected from the $\mathrm{K}$ shell. There are different notations and formalisms in describing the involved atomic transitions depending on the excited electrons and their quantum numbers. Two conventional types of notation are known as the "atomic notation" and the "x-ray notation". They are presented for the first few atomic levels in Table 4.1. 


\subsection{Introduction}

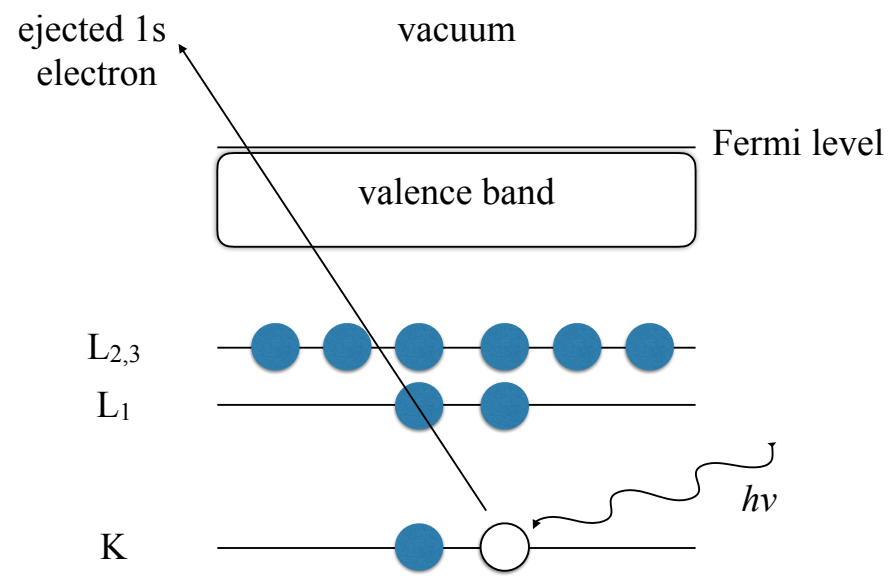

Figure 4.2: Schematic presentation of the core $1 s$ XPS process

\begin{tabular}{|c|c|c|c|c|}
\hline$n$ & $l$ & $j$ & Atomic Notation $\left(n l_{j}\right)$ & X-ray Notation \\
\hline 1 & 0 & $1 / 2$ & $1 s_{(1 / 2)}$ & $K_{1}$ \\
\hline 2 & 0 & $1 / 2$ & $2 s_{(1 / 2)}$ & $L_{1}$ \\
\hline 2 & 1 & $1 / 2$ & $2 p_{(1 / 2)}$ & $L_{2}$ \\
\hline 2 & 1 & $3 / 2$ & $2 p_{(3 / 2)}$ & $L_{3}$ \\
\hline 3 & 0 & $1 / 2$ & $3 s_{(1 / 2)}$ & $M_{1}$ \\
\hline 3 & 1 & $1 / 2$ & $3 p_{(1 / 2)}$ & $M_{2}$ \\
\hline 3 & 1 & $3 / 2$ & $3 p_{(3 / 2)}$ & $M_{3}$ \\
\hline 3 & 2 & $3 / 2$ & $3 d_{(3 / 2)}$ & $M_{4}$ \\
\hline 3 & 2 & $5 / 2$ & $3 d_{(5 / 2)}$ & $M_{5}$ \\
\hline
\end{tabular}

Table 4.1: The relation between quantum numbers, atomic notation and x-ray notation for the first few energy levels [3. $j$ is the total angular momentum quantum number which takes $|\ell-s| \leq j \leq|\ell+s|$ values where $s=1 / 2$ for electrons. 


\subsection{Introduction}

\subsubsection{XPS detection technique}

The probability of interaction with the matter is much smaller for photons than for electrons, and that is why the detection depth is much bigger for the photon-in photon-out techniques (such as XAS and RXES) than for the electron-out techniques. The detection depth for such photon-out techniques is of the order of micrometers while it is only of the order of tens of angstroms for the electron-out ones such as XPS. This makes XPS a very surface sensitive technique which means it is crucial to have a clean sample surface to get a reliable spectrum.

The detection depth of XPS can be determined by a quantity known as the attenuation length $(\lambda)$ of the electrons under study. $\lambda$ depends on the kinetic energy of the electrons and also the properties of the material [3]:

$$
\lambda=\frac{538 a_{A}}{E_{A}^{2}}+0.41 a_{A}\left(a_{A} E_{A}\right)^{0.5},
$$

where $a_{A}^{3}$ is the volume of the atom in $\mathrm{nm}^{3}, E_{A}$ is the energy of the electron in $\mathrm{eV}$ and $\lambda$ is in $\mathrm{nm}$.

Following the Beer-Lambert equation, the intensity of electrons $(I)$ ejected at an angle $\theta$ to the surface normal is given by:

$$
I=I_{0} e^{-\frac{d}{\lambda \cos \theta}}
$$

where $I_{0}$ is the electron intensity from an infinite and uniform substrate.

Further analysis of the above equation gives that 65 percent of the detected signals in electron spectroscopy come from a depth less than $\lambda, 85$ percent from a depth less than $2 \lambda$ and 95 percent from a depth less than $3 \lambda$. The angle of the sample to the detector can be varied to change the detection depth. A sample mounted at an angle normal to the analyzer axis is probed deeper than the one with a more shallow electron take-off angle [3, 15] as demonstrated in Fig. 4.3.

\subsubsection{Theory}

In this section, we explain the photoionization phenomenon in a simplified single particle picture [17, 84]. This description can, in fact, be very useful in understanding the XPS process especially when there are no strong electronic correlations. Very similar to what was explained in chapter 3 for the x-ray absorption theory, the most general approach of the photoemission theory is also based on the Fermi's Golden rule derived from the time-dependent perturbation theory. In this approach the XPS current or 


\subsection{Introduction}

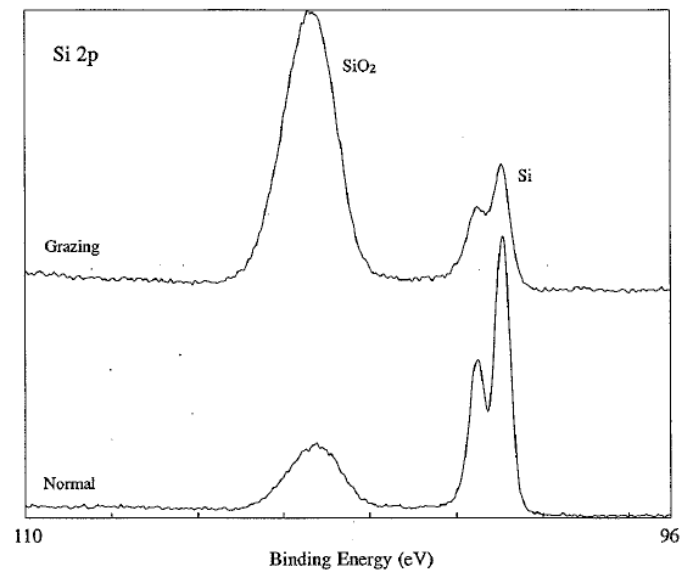

Figure 4.3: This shows how at a low electron take off angle (grazing) the surface sensitivity is enhanced and the thin oxide layer on the silicon is more dominant [15].

the transition rate from an initial state $\left|\psi_{i}\right\rangle$ to a final state $\left|\psi_{k, s}\right\rangle$ is given by:

$$
I_{k}(h \nu)=\frac{2 \pi}{\hbar} \sum_{s}\left|<\psi_{k, s}\right| H^{1}\left|\psi_{i}>\right|^{2} \delta\left(\epsilon_{k}-\epsilon_{s}-h \nu\right),
$$

where the final state $\left|\psi_{k, s}\right\rangle$ is the state with the photoelectron of momentum $k$ and energy $E_{k}=\frac{\hbar^{2} k^{2}}{2 m_{e}}$ and a system with the remaining $N-1$ electrons. The index $s$ is the set of quantum numbers of all the possible final states. $H^{1}$ is also the perturbing potential describing the interaction of electrons in an electromagnetic field. As explained in chapter $3, H^{1}$ can be given by:

$$
H^{1}=\frac{e}{m_{e} c} \vec{A} \cdot \vec{p}
$$

where $\vec{p}$ is the momentum operator and $\vec{A}$ is the electromagnetic vector potential.

Now in order to proceed with the calculation, an approximation,namely the sudden approximation, has to be made. This approximation allows us to decouple the photoelectron from the remaining $N$-1-electron system in the final state. Therefore, in Eq. 4.4 we can substitute the $\left|\psi_{k, s}\right\rangle$ state 


\subsection{Introduction}

with $c_{k, s}^{\dagger} \mid N-1, s>$ where $c_{k, s}^{\dagger}$ is the creation operator for the photoelectron. With this approximation, Eq. 4.4 becomes:

$$
I_{k}(h \nu)=\frac{2 \pi}{\hbar} \sum_{k}\left|\Delta_{\kappa k}\right|^{2} A_{k}^{<}\left(\epsilon_{\kappa}-h \nu\right)
$$

where $\Delta_{\kappa k}=\left|\left\langle\psi_{\kappa}\left|H^{1}\right| \psi_{k}\right\rangle\right|$ are the photoemission transition probability matrix elements, and $A_{k}^{<}(h \nu)=\sum_{s}|<N-1, s| c_{k}|N>|^{2} \delta\left(h \nu-\epsilon_{s}\right)$ is known as the single particle spectral function. Eq. 4.6 can also be written in terms of the Green's function as the spectral function $A$ is related to the Green's function $G$ as:

$$
A(k, \omega)=\frac{1}{\pi}|\operatorname{Im} G(k, \omega)|
$$

\subsubsection{XPS, core levels and satellites}

A key advantage of XPS is its ability to identify the elements based on their binding energies and chemical shifts of the photoemission lines. In a simple $\mathrm{K}$ shell XPS process a core $1 s$ electron is excited by an incident photon, and its kinetic energy is measured and thereby its binding energy is found. A typical result of such measurement is shown in Fig. 4.4 for elements of the second row of the periodic table from Li to F. As shown in the figure, the XPS spectra consist of a line at a binding energy that expectedly increases with the atomic number.

An XPS peak can also exhibit a multiplet splitting in its structure. This splitting arises in a $1 s$ XPS peak when the compound contains unpaired electrons in its valence band. Multiplet structure results from the exchange interaction which can only be operative if the electrons in the valence shell are not all paired, i.e., if there is a net local spin moment ${ }^{2}$, A core hole is created through the photoionization process which can couple with the unpaired valence electron. This can create multiple available final states at different energies resulting in the multiplet splitting of the main peak. This is shown in Fig. 4.5 where the oxygen core $1 s$ XPS of $\mathrm{H}_{2} \mathrm{O}$ is compared to the $\mathrm{O}_{2}$ gas. Diamagnetic $\mathrm{H}_{2} \mathrm{O}$ has a closed-shell configuration with all its electrons paired and a total spin of zero. Therefore, the coupling between

\footnotetext{
${ }^{2}$ Multiplet structure can also occur for systems with all paired electrons but a not completely filled valence shell and with very small bandwidths so that the valence shell electrons on the atom with the core hole are bound to the core hole. Now, one has two open shells, and this causes multiplet structure not only because of the exchange interaction but due to the higher multiple Coulomb interactions between the core hole and the valence electrons.
} 


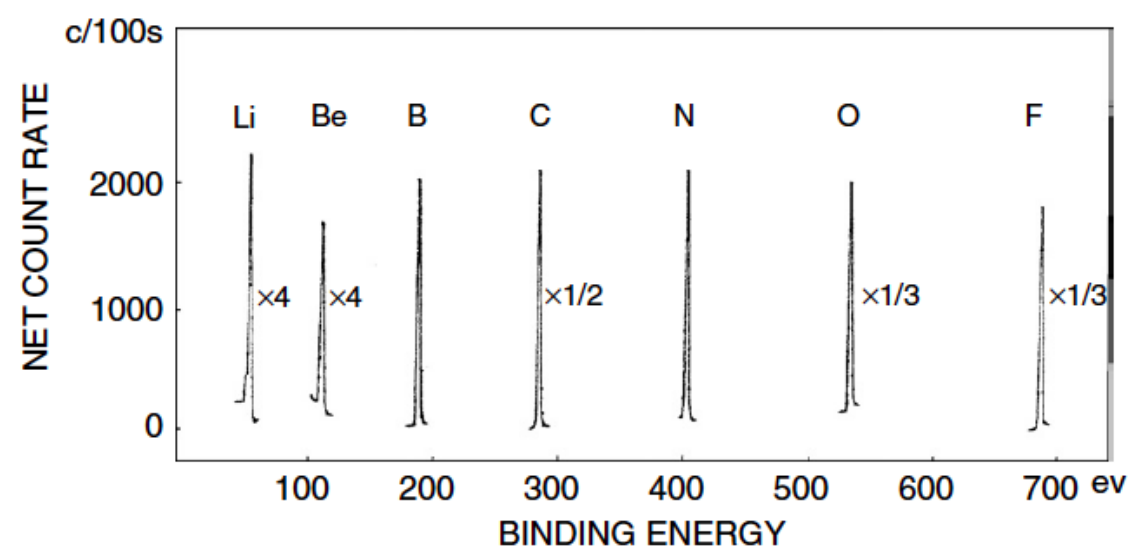

Figure 4.4: Core 1s XPS spectra of elements with the atomic numbers of 3 to 9 . The binding energy increases with increasing the atomic number $[16,17]$.

the spin up and spin down core hole to the valence electrons gives degenerate states with the same energy. Thus, its XPS spectrum consists of a single peak. However, $\mathrm{O}_{2}$ is paramagnetic with two unpaired electrons in its $\pi^{\star} 2 p$ orbitals. The core-hole (or the remaining single electron) created through the XPS, can be either parallel or antiparallel to the valence electrons resulting in two different non-degenerate final states with an energy difference of $\Delta E=1.1 \mathrm{eV}$. This type of splitting is called multiplet splitting. They can give additional rich information regarding the fine structure of the atoms and their chemical environment [17, 18].

Shake-up satellites are another possible source of additional lines in an XPS spectrum. These can occur when the ejected photoelectron interacts with a valence electron and gives some of its kinetic energy to excite the compound to a higher energy state. This will be detected as an additional line at a lower kinetic energy or a higher binding energy than the main peak and is called a "shake up" peak or satellite.

\subsection{XPS Results}

We employed XPS as one of the most powerful spectroscopy techniques to study our material of interest $\mathrm{BaBiO}_{3}$. As mentioned before, we are particularly interested in investigating the role of ligand oxygens in the electronic 


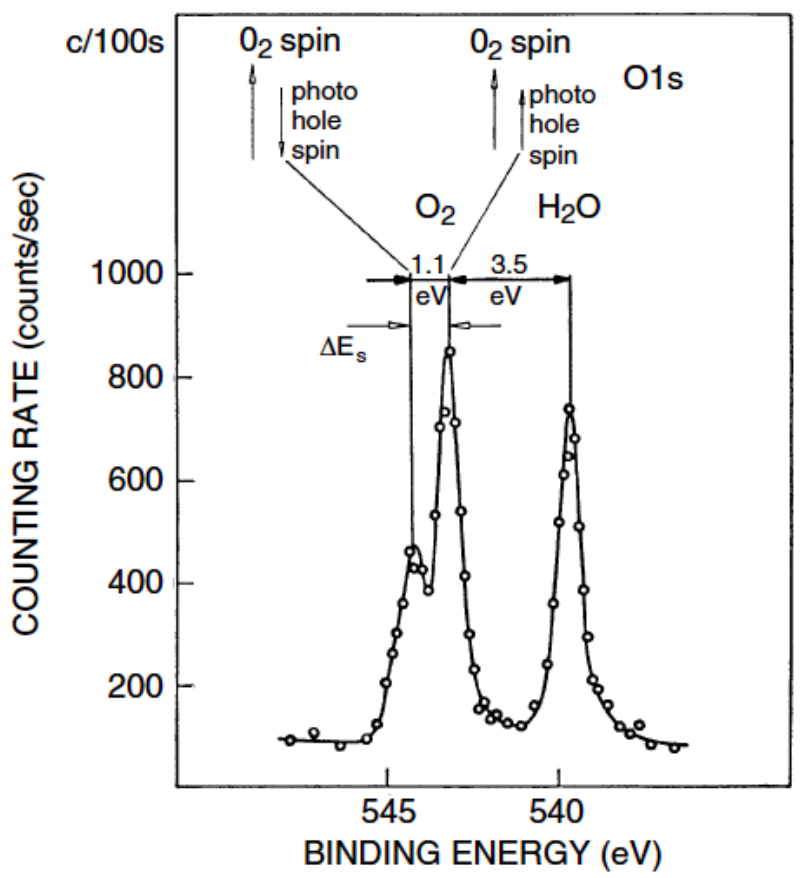

Figure 4.5: Oxygen $1 s$ XPS of $\mathrm{O}_{2}$ and $\mathrm{H}_{2} \mathrm{O}$ in their gas phase. $\mathrm{O}_{2}$ is paramagnetic with two unpaired electrons in its valence shell. The core-hole created through the XPS process can interact with these and gives two nondegenerate states observed as the two XPS lines. $\mathrm{H}_{2} \mathrm{O}$ is diamagnetic with all its valence electrons paired; therefore, it gives one single XPS peak. [17, 18, 
behaviour of the material. We achieved this partly by doing oxygen K-edge XAS and RXES on our single crystal in chapter 3 . They confirmed the existence of a high hole density in the oxygens ground state consistent with the band structure calculation results and the proposed bond disproportionation insulating mechanism.

However, this result, in turn, poses the following question: If these corelevel experiments give evidence for a high oxygen $p$ hole density, one should be able to see that evidence in an oxygen $1 s$ XPS measurement, too. Within this bond-disproportionation picture, there have to be two holes per octahedron or $1 / 3$ hole per oxygen on average. Therefore, due to the strong Coulomb interaction between this $1 / 3$ hole and the $\mathrm{O} 1 \mathrm{~s}$ core-hole, created through the XPS, one would generally expect to see a main peak and a strong satellite structure in the oxygen $1 s$ XPS spectrum. However, while many find such structure, they assign it merely to the surface contamination $[28,31,34]$ and avoid explaining the oxygens' electronic role in the spectrum. Moreover, on the other hand, some assign this structured peak to the different oxidation states of the oxygens without perhaps the required diligent cleaning procedure $[30$.

In this work, to address this problem, we start by cleaning our highquality single crystals systematically by monitoring the carbon peak and the corresponding oxygen peak simultaneously. we then enhance the cleaning procedure accordingly. In the following sections, each cleaning stage is explained, and the corresponding result is shown.

\section{First Attempt}

Cleaving a crystal is a well-known solution to expose a clean surface for an XPS experiment. Cleaving often works nicely for "two-dimensional" materials such as cuprates where the layers are bonded only through a weak Van der Waals force. However, since $\mathrm{BaBiO}_{3}$ is a three-dimensional material after trying multiple times, we found that the crystal does not cleave but instead fractures with a very rough surface at best. In most attempts at cleaving, the crystal itself was removed with nothing left on the sample holder. There does not seem to be a clear cleavage plane consistent with the three-dimensional crystal structure.

Therefore, for our first attempt, we cleaned the single crystal with acetone and deionized water in an ultrasonic bath for 10 minutes each. Then we dried the surface using nitrogen flow. Next, the sample was heated ("baked") in vacuum at $100^{\circ} \mathrm{C}$ for 20 minutes in the "transfer chamber", before being transferred to the ultra-high vacuum XPS chamber. These measurements 

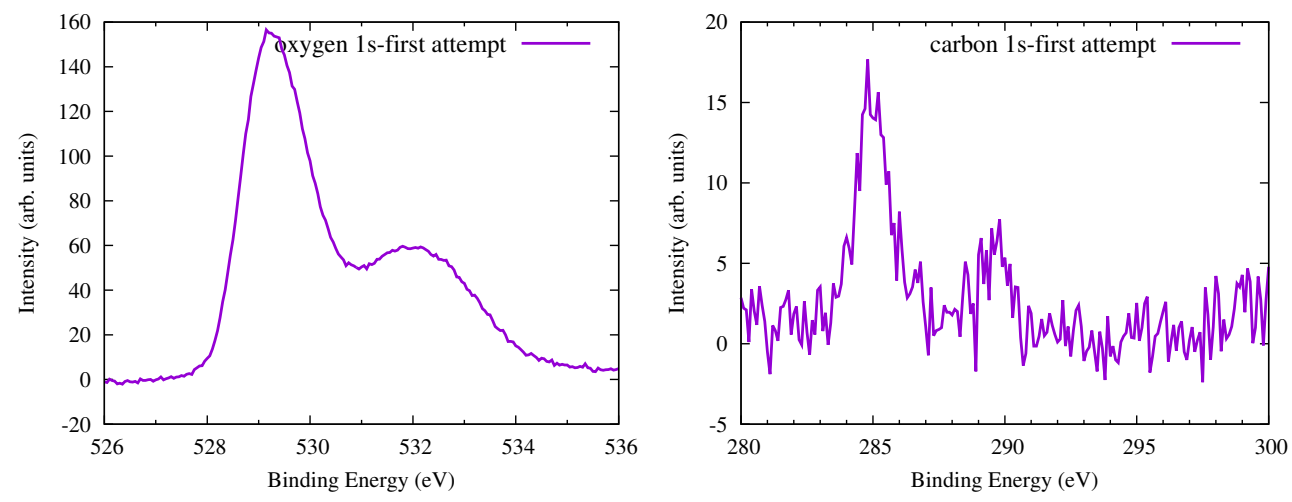

Figure 4.6: Oxygen 1s and carbon 1s XPS spectra after our first attempt in cleaning the surface of the single crystal $\mathrm{BaBiO}_{3}$.

were done at the Surface Science Facility of the REIXS beam-line at the CLS. XPS was collected using a monochromatic x-ray source corresponding to the aluminium $K_{\alpha}$ emission line at $1486.6 \mathrm{eV}$ with an energy resolution of $0.4 \mathrm{eV}$. All of the measurements were done at the normal electron take-off angle for the least surface sensitivity. The specifications of the XPS system is given in chapter 2.3.1.

For each attempt, a wide-range scan, an oxygen $1 s$, and a carbon $1 s$ scan were done. The wide-range scans were used to make sure the stoichiometry of the crystal does not change with the cleaning procedure. The oxygen and carbon $1 s$ spectra after this preliminary cleaning step are shown in Fig. 4.6. As it can be seen, the oxygen spectrum has some significant shoulder at a higher binding energy, but the carbon peak also indicates the presence of some surface contaminations. Therefore, it is not possible to conclude if the oxygens structure is intrinsic or extrinsic since the high binding energy peak in $\mathrm{O} 1 s$ spectra is well known to be indicative of $\mathrm{H}_{2} \mathrm{O}$ or $\mathrm{CO}_{2}$ contamination of the surface.

\section{Second Attempt}

After the previous attempt, we noticed under the optical microscope, that the acetone damages the surface by leaving some coppery stains on the golden surface of the crystal. Therefore, we opted for heptane which is known as a less destructive cleaning solvent. We repeated the previous cleaning steps but with heptane instead of acetone and on a new single crystal (but from the same batch). The result is shown in Fig. 4.7 and is 
compared to the previous result. As shown, here we get a different carbon $1 s$ spectrum and also a different oxygen $1 s$ peak. This is an indication that the oxygen XPS structure is at least partly due to the surface contamination and we indeed need to take good care of our cleaning steps.

\section{Third Attempt}

Here, as our third modification to the cleaning procedure, we repeated the "second attempt" cleaning steps but we increased the "baking" time from 20 minutes to 40 minutes. The result is shown in Fig. 4.7 and is compared to the previous measurements. As it can be seen, the carbon peak has lost its intensity indicating that increasing the baking time helps with removing the carbon-containing surface contaminations.

\section{Fourth Attempt}

At this point, we decided to add another step to enhance our cleaning procedure further. For our fourth attempt, after all the steps taken previously, we transferred the crystal to an ultra-high vacuum chamber with the base pressure of less than $1 \times 10^{-10}$ Torr. There, it was annealed at $200^{\circ} \mathrm{C}$ for 1 hour in $1.7 \times 10^{-7}$ Torr oxygen atmosphere with a strong atomic oxygen component using a $55 \mathrm{~W}$ power thermal gas cracker. Atomic oxygen is highly oxidizing and helps oxidizing any carbon-containing molecules physisorbed to the surface. It also protects the sample against oxygen deficiency. The result after this procedure is shown in Fig. 4.7. As shown, this step was quite successful in removing the carbon contribution.

\section{Fifth Attempt}

After we found that annealing in the atomic oxygen is very effective in removing the carbon-containing contaminations, we decided to repeat the procedure but at a higher temperature of $350^{\circ} \mathrm{C}$ rather than $200^{\circ} \mathrm{C}$. The result is shown in Fig. 4.7. It can be seen that this, in fact, improved the surface cleaning by removing the carbon contamination even further.

\section{Final Attempt}

We managed to remove the carbon peak to a large extent so far. We also found out the structure of the oxygen peak is affected by the surface contamination significantly. However, there are still some carbon-containing 


\subsection{XPS Results}
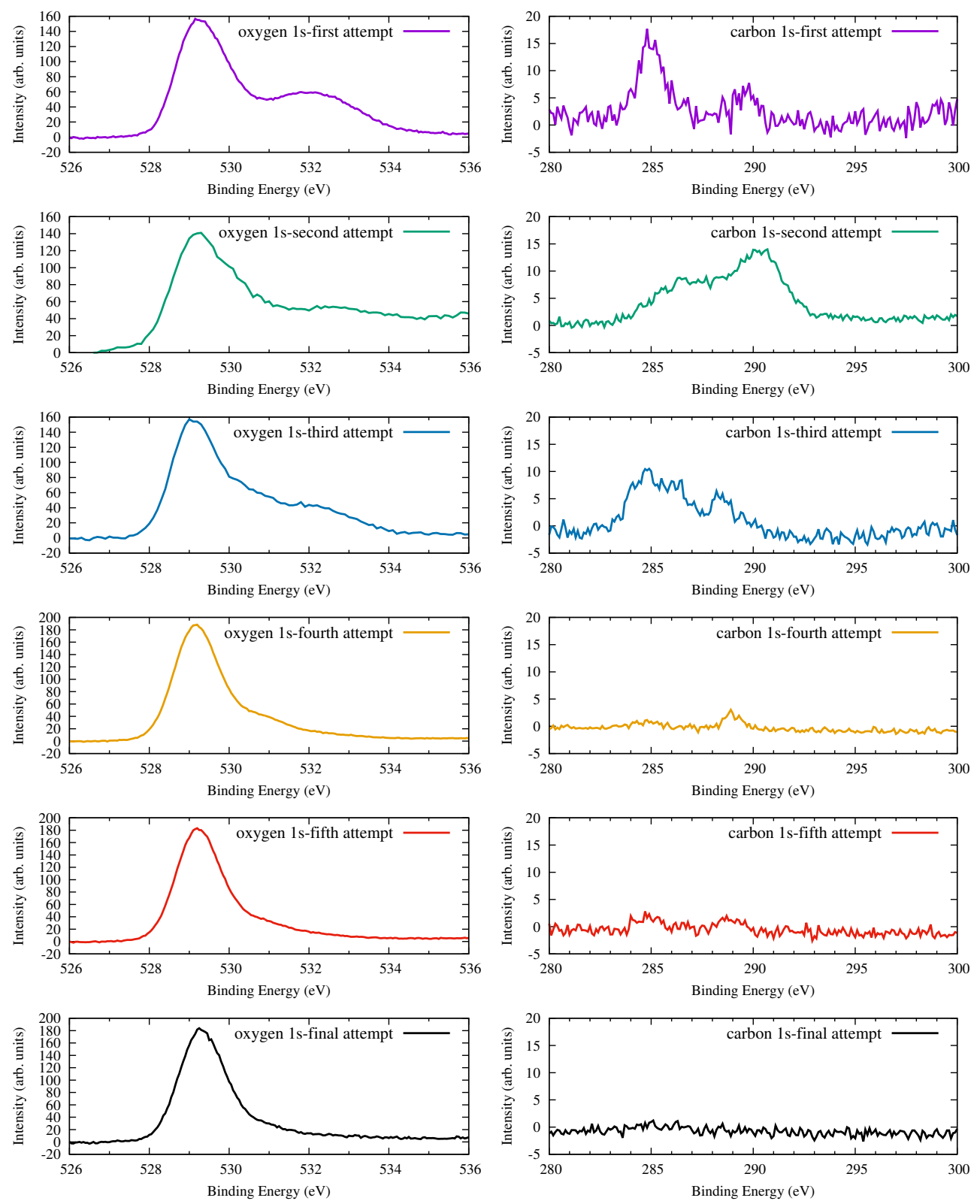

Figure 4.7: Oxygen $1 s$ and carbon $1 s$ XPS spectra at different stages of our cleaning procedure on the single crystal $\mathrm{BaBiO}_{3}$. The removal of the carbon peak confirms the effectiveness of the procedure. 

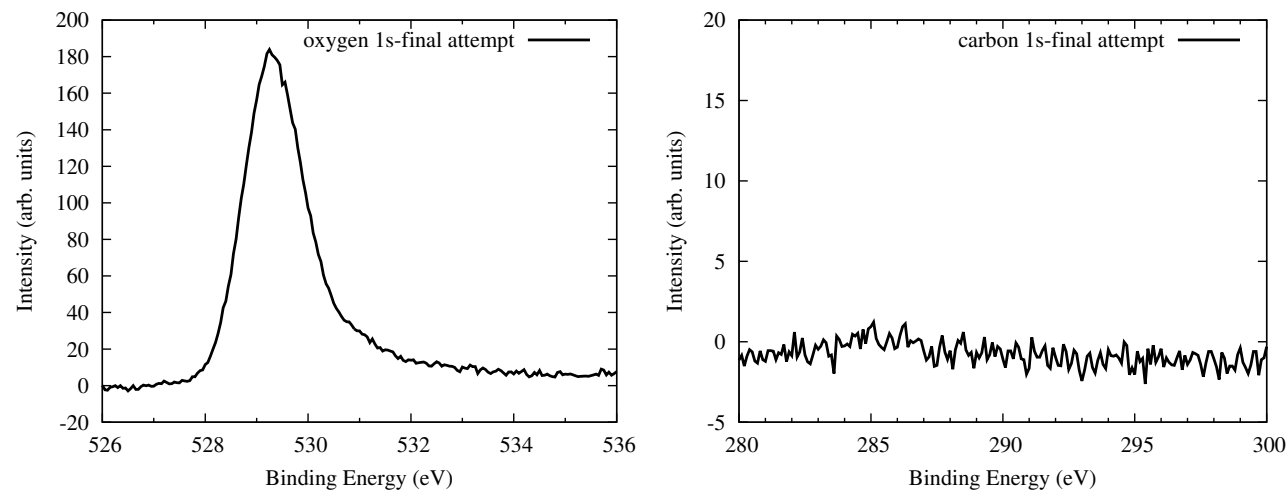

Figure 4.8: Oxygen $1 s$ and carbon $1 s$ XPS spectra of single crystal $\mathrm{BaBiO}_{3}$ after the optimized cleaning procedure described in the text. The oxygen spectrum loses most of its structure with the progression of the cleaning but remains slightly asymmetric.

contamination remained that we wish to remove. To achieve that, we repeated the previous steps but did the atomic oxygen annealing step longer for two hours rather than one.

The result along with the result from the previous attempts are shown in Fig. 4.7. As shown, we finally managed to remove the carbon peak ,i.e., all the carbon-containing surface contaminations from the surface perfectly. The assumption then is that the non-carbon-containing molecules such as $\mathrm{H}_{2} \mathrm{O}$ are also desorbed during this whole process.

One important observation through this whole process was how the structure of the oxygen spectrum was affected by removing the carbon. At the end, it lost much of its structure but remained slightly asymmetric as shown in Fig 4.8. It confirms its satellite structure was mainly due to the surface contamination and not intrinsic to the material. This result is rather surprising at first given the previous XAS+RXES results indicating a high hole density on the oxygens. As mentioned before, one would have expected to see a strong satellite structure in an oxygen $1 s$ XPS due to this oxygen hole density. We address this question in the next chapter where we calculate the $\mathrm{BaBiO}_{3}$ oxygen $1 s$ XPS using exact diagonalization cluster calculation. 


\subsection{Conclusion}

\subsection{Conclusion}

We studied single crystal $\mathrm{BaBiO}_{3}$ using oxygen $1 s$ XPS technique. We showed surface contamination could be a serious issue in interpreting the structure of the oxygen spectrum. We managed to develop a successful cleaning routine to remove the carbon-containing compounds from the surface. Annealing at atomic oxygen atmosphere was found highly effective in this routine. We showed by removing the surface contamination oxygen $1 s$ spectrum also loses much of its structure and its shoulder but remains slightly asymmetric. 


\section{Chapter 5}

\section{Exact Diagonalization Cluster Model}

In this chapter, we use the cluster calculation method to investigate the electronic structure of $\mathrm{BaBiO}_{3}$ and also to calculate its oxygen $1 s$ XPS to understand the experimental results shown in the previous chapters. In chapter 3 we discussed how the oxygen K-edge XAS+RXES confirms the presence of a high oxygen hole density in the material. We then in chapter 4 showed the oxygen $1 s$ XPS results on a clean single crystal. Due to the strong Coulomb interaction between the XPS core-hole and the assumed hole density on the oxygens one would have expected to see a strong satellite structure in the oxygen $1 s$ XPS. However, we showed the observed shoulder of the main peak is virtually only due to the surface contamination and the XPS of a clean surface does not have any strong satellite or multiplet structure. Now in this chapter, we use the exact diagonalization cluster model to address this problem.

\section{$5.1 \quad$ Introduction}

As discussed in the previous chapters, in order to calculate various x-ray spectra one needs to have the initial and the final many-body quantum states of the $\operatorname{system}\left(\psi_{i}\right.$ and $\left.\psi_{f}\right)$. In order to get these states, one has to solve the Schrdinger equation 5.1 with a given Hamiltonian.

$$
H \psi=E \psi
$$

Solving the Schrodinger equation with the real Hamiltonian of a manybody system is nearly impossible. However, one can make some approximations and construct a more manageable model Hamiltonian and still extract much information about the system by solving that. Depending on the complexity of the system, the desired result, and the available computational power, these model Hamiltonians can vary in their levels of complexity. Below we introduce some of these approaches and their model Hamiltonians in 


\subsection{Introduction}

an increasing order of complexity.

\subsubsection{Atomic Model}

For a non-relativistic time-independent atomic model, where electrons and the nucleus are treated as point charges, the Hamiltonian of a system with $j$ electrons and a nucleus with an atomic number of $\mathrm{Z}$ looks like:

$$
H_{\text {Coulomb }}=T_{e}+U_{e-n}+U_{e-e}=-\frac{\hbar^{2}}{2 m_{e}} \sum_{j} \nabla_{j}^{2}-\sum_{j} \frac{Z e^{2}}{r_{j}}+\sum_{j>k} \frac{e^{2}}{r_{j k}}
$$

where $T_{e}$ is the kinetic energy of the electrons with the effective mass of $m_{e} . U_{e-n}$ is the Coulomb interaction between the electrons and the nucleus where $r_{j}$ is the distance between the nucleus and the electron $j . U_{e-e}$ is the Coulomb interaction among the electrons where $r_{j k}$ is the distance between the electron $j$ and the electron $k$ [85]. Here the interaction terms due to the electronic and nuclear spin are neglected. Although this Hamiltonian makes major approximations in treating the system, it is still impossible to solve analytically for molecules with more than a few electrons. However, thanks to the modern computational power, some self-consistent iterative approaches can give reasonably accurate answers for single atoms [11, 86.

Almost all approaches to solving the Coulomb Hamiltonian are based on the Born-Oppenheimer (BO) approximation which is based on the assumption that the electronic and the nuclear parts of the Hamiltonian can be separated. Consequently, the wave-function of the total Hamiltonian can be written in terms of the nuclear and the electronic wave-functions as:

$$
\psi_{\text {tot }}=\psi_{\text {electronic }} \times \psi_{\text {nuclear }}
$$

One step further in the "atomic model" is considering the interaction between the spin and angular motion of the electrons via the spin-orbit coupling term. This can be written as:

$$
H_{\text {atomic }}=H_{\text {Coulomb }}+H_{S O}=H_{\text {Coulomb }}+\sum_{j} \xi_{j}\left(r_{j}\right)\left(l_{j} . s_{j}\right)
$$

where $l_{j}$ and $s_{j}$ are the orbital and spin angular momentum operators of the electron $j$, and $\xi$ is the spin-orbit coupling constant. For an electron moving in a central potential $U(r), \xi$ can be expressed as $\xi=\frac{\hbar^{2}}{2 m^{2} c^{2}} \frac{1}{r} \frac{\delta U(r)}{\delta(r)}$ [85]. 


\subsection{Introduction}

The Pauli principle states no two electrons can occupy the same spin orbitals. In other words, all the wave-functions must be anti-symmetrical under exchange of any two of the electrons ( or more generally fermions). This will result in the wave-functions of the form of a normalized "Slater determinant" which satisfies the antisymmetric properties of a fermionic wave-function:

$$
\Psi\left(\mathbf{x}_{1}, \mathbf{x}_{2}, \ldots, \mathbf{x}_{N}\right)=\frac{1}{\sqrt{N !}}\left|\begin{array}{cccc}
\chi_{1}\left(\mathbf{x}_{1}\right) & \chi_{2}\left(\mathbf{x}_{1}\right) & \cdots & \chi_{N}\left(\mathbf{x}_{1}\right) \\
\chi_{1}\left(\mathbf{x}_{2}\right) & \chi_{2}\left(\mathbf{x}_{2}\right) & \cdots & \chi_{N}\left(\mathbf{x}_{2}\right) \\
\vdots & \vdots & \ddots & \vdots \\
\chi_{1}\left(\mathbf{x}_{N}\right) & \chi_{2}\left(\mathbf{x}_{N}\right) & \cdots & \chi_{N}\left(\mathbf{x}_{N}\right)
\end{array}\right|
$$

where the many-electron wave-function $\Psi\left(\mathbf{x}_{1}, \mathbf{x}_{2}, \ldots, \mathbf{x}_{N}\right)$ is expressed in terms of $\chi_{i}\left(\mathbf{x}_{i}\right)$ s which form a complete orthonormal basis set of wavefunctions for single electrons.

The next step in treating the "atomic model" is writing the electronelectron interaction potential matrix elements with wave-functions of the above form $\left(<\Psi\left|\sum_{j>k}^{N} \frac{e^{2}}{r_{j k}}\right| \Psi>\right)$. This will lead to the formation of socalled Slater integrals $\left(F^{k}(i, j)\right)$ corresponding to the Coulomb and exchange interactions between electron pairs. Thus, the Slater integrals are not defined according to the total many-body wave function but are the Coulomb integrals describing the average interaction between two electrons in these respective one-particle wave functions. These energies are the main empirical values used in this model [85, 87. However, one has to remember these energies are often strongly screened and reduced in solids due to the polarization effects $[80,88]$.

In summary, in this model Hamiltonian, we assume that the atomic oneelectron wave functions remain unchanged in the solid and that the wave function of electrons moving in the solid is a result of a linear combination of atomic wave functions centred on the atoms of the solid. In quantum chemistry, this is called the linear combination of atomic orbitals method, and in solid state physics, these would result in the so-called tight binding wave functions describing the band structure of the electrons in the solid. While this model gives valuable insight into the basic physics of a manyelectron system, one has to go further to extract truly useful and practical information about a real condensed matter system. 


\subsection{Introduction}

\subsubsection{Crystal Field Model}

The basic idea in the crystal field model is that the atom of interest is subjected to an electrostatic field produced by its surrounding ions or ligands. In this model, the ligands are treated as point charges. The key idea of this model was discussed by Becquerel in 1929 [89], and was formulated into a rigorous theory by Bethe in the same year 85,90 . Bethe showed how the symmetry and the strength of this electrostatic potential could affect the energy levels of an ion. In 1932, Van Vleck successfully demonstrated the capability of this theory by explaining "why nickel salts are nearly isotropic magnetically, while those of cobalt exhibit over 25 percent anisotropy even though the $\mathrm{Ni}^{++}$and $\mathrm{Co}^{++}$ions are both in $\mathrm{F}$ states and are adjacent in the periodic table" [91].

In this model, the role of ligands is somewhat limited since their electrons cannot hybridize or overlap with each other or the metal ion. Therefore, its Hamiltonian can be easily modelled by adding a perturbing electrostatic potential $V$, the potential created by ligands, to the Hamiltonian of the free ion:

$$
H_{C F}=H_{\text {atomic }}+V
$$

Note that $V$ must have the same symmetry as the symmetry group of the molecule because the Hamiltonian of the system must remain invariant under all symmetry operations [85]. This model finds the energy levels of the system to be very sensitive to the geometry of the ligands.

Neglecting the spin-orbit coupling for the moment, Fig. 5.1 shows how the crystal field with different symmetries affect the five $d$ orbitals. The reason is simply that the $d$ electrons want to avoid the regions with the highest electron density from the ligands to lower the Coulomb repulsion energy. That is why the $d$ orbitals, which are all degenerate in a spherical potential $\left(\mathrm{SO}_{3}\right)$, split into two different energy levels in an octahedral symmetry $\left(\mathrm{O}_{h}\right)$ namely the $e_{g}$ and $t_{2 g}$ orbitals. $e_{g}$ orbitals are the ones pointing directly towards the vertices of the octahedron where the ligands with their negative charges reside. Thus, they will have higher energy than the other three orbitals $\left(t_{2 g}\right)$ that are located off-axis and therefore have a lower energy [92]. The energy difference between these two is usually known as $10 D q$. The spin-orbit coupling can split the energy levels even further. 


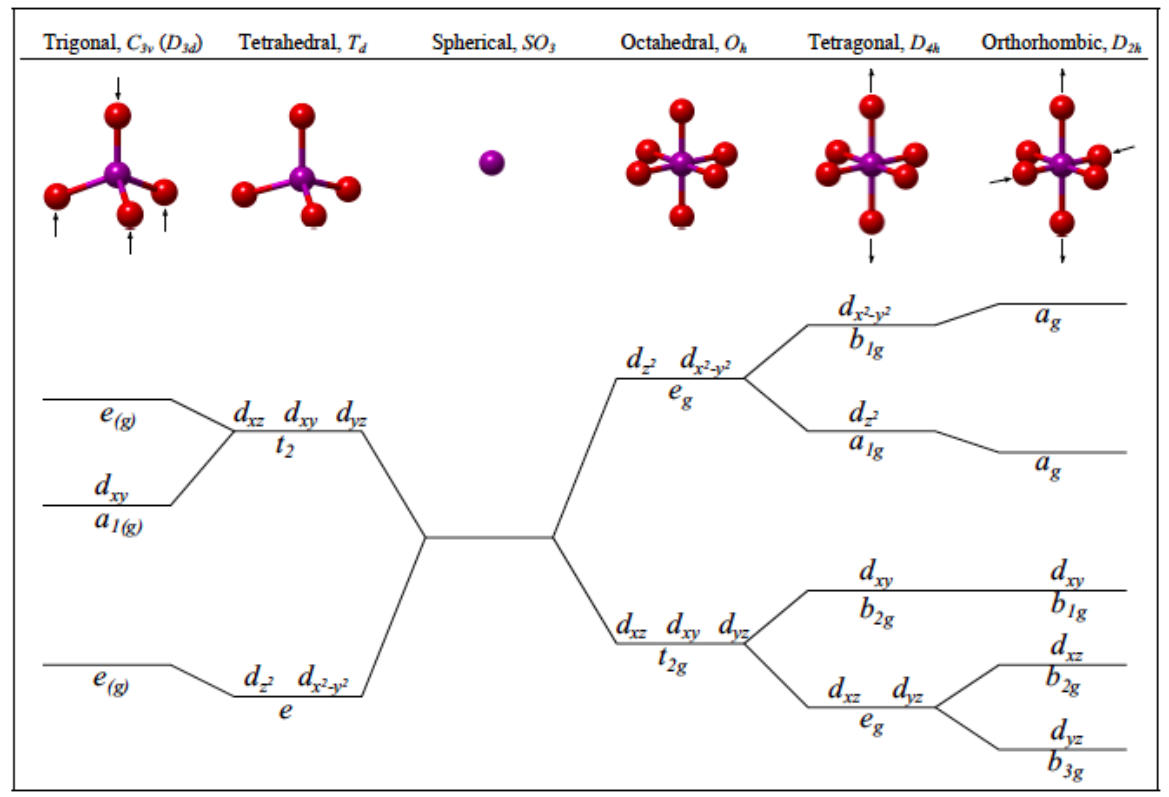

Figure 5.1: $d$ orbitals in various crystal field symmetries. The specific symmetry of the ligands affect the energy levels of the metal ion [11]. 


\subsection{Introduction}

\section{Hund's rules}

It was mentioned how a crystal field can split the orbitals' energy levels and lift the degeneracy. The Hund's rules, introduced by Friedrich Hund around 1925, dictate how these orbitals are filled in the ground state [93]:

- The first rule is also known as the "Hund's rule of maximum multiplicity" where multiplicity is defined as $2 S+1$ where $S$ is the total spin. This rule implies the total spin $S$ has to be maximized in the ground state. In other words, the number of parallel spin pairs has to be maximized. From the Pauli exclusion principle, this requires electrons to be in different spatial orbitals which minimizes the Coulomb repulsion and lowers the energy.

- For a given multiplicity, the second rule requires the total orbital angular momentum to be maximized for the ground state. This also results in a lower Coulomb repulsion and minimizes the energy.

- And finally the third rule says that for the less-than-half filled outer shells, the state with the minimum total angular momentum quantum number $(J=L-S)$ is the ground state while for the more-than-half filled shells, the level with the maximum total angular momentum quantum number $(J=L+S)$ becomes the lowest energy state.

The Hund's ground state energy for a system with $n$ electrons is determined by (neglecting spin orbit coupling) :

$$
E(n, H \text { und })=\alpha_{I}(n) I+\alpha_{F}^{0}(n) F^{0}+\alpha_{J}(n) J+\alpha_{C}(n) C
$$

where $I$ is the one-electron potential, $F^{0}$ is the monopole part of the Coulomb integral, $C$ describes the angular part of the multiplet splitting and $J$ is the Hund's exchange interaction energy. $\alpha_{I}=n, \alpha_{F}^{0}(n)=\frac{n !}{2}$, and $\alpha_{J}(n)$ is the number of parallel spin pairs $[92,94,95]$.

As an example, the arrangements of $d$ electrons are presented in Table. 5.2 in an octahedral complex following the above Hund's rules [96]. Note that depending on the Hund's exchange interaction energy $(J)$ and the onsite energies of the orbitals, states with a low spin can have a lower Hund's ground state energy than the high spin state like the $4 d$ electrons in a weak crystal field potential versus in a strong crystal field potential. 


\begin{tabular}{|c|cc|c|cc|c|}
\hline $\begin{array}{c}\text { Number of } d \\
\text { electrons }\end{array}$ & \multicolumn{2}{|c|}{$\begin{array}{c}\text { In weak } \\
\text { crystal field }\end{array}$} & $\mathbf{N}$ & \multicolumn{2}{|c|}{$\begin{array}{c}\text { In strong } \\
\text { crystal field }\end{array}$} & $\mathbf{N}$ \\
\hline 1 & $\begin{array}{c}t_{2 g} \\
\uparrow\end{array}$ & $-e_{g}$ & 0 & $\begin{array}{l}t_{2 g} \\
e_{g}\end{array}$ & 0 \\
\hline 2 & $\uparrow \uparrow$ & - & 1 & $\uparrow \uparrow$ & - & 1 \\
\hline 3 & $\uparrow \uparrow \uparrow$ & - & 3 & $\uparrow \uparrow \uparrow$ & - & 3 \\
\hline 4 & $\uparrow \uparrow \uparrow$ & $\uparrow$ & 6 & $\uparrow \downarrow \uparrow \uparrow$ & - & 3 \\
\hline 5 & $\uparrow \uparrow \uparrow$ & $\uparrow \uparrow$ & 10 & $\uparrow \downarrow \uparrow \downarrow \uparrow$ & - & 4 \\
\hline 6 & $\uparrow \downarrow \uparrow \uparrow$ & $\uparrow \uparrow$ & 10 & $\uparrow \downarrow \uparrow \downarrow \uparrow \downarrow$ & - & 6 \\
\hline 7 & $\uparrow \downarrow \uparrow \downarrow \uparrow$ & $\uparrow \uparrow$ & 11 & $\uparrow \downarrow \uparrow \downarrow \uparrow \downarrow$ & $\uparrow$ & 9 \\
\hline 8 & $\uparrow \downarrow \uparrow \downarrow \uparrow \downarrow$ & $\uparrow \uparrow$ & 13 & $\uparrow \downarrow \uparrow \downarrow \uparrow \downarrow$ & $\uparrow \uparrow$ & 13 \\
\hline 9 & $\uparrow \downarrow \uparrow \downarrow \uparrow \downarrow$ & $\uparrow \downarrow \uparrow$ & 16 & $\uparrow \downarrow \uparrow \downarrow \uparrow \downarrow$ & $\uparrow \downarrow \uparrow$ & 16 \\
\hline
\end{tabular}

Figure 5.2: The arrangement of $d$ electrons in the ground state of an octahedral complex following the Hund's rules. $N$ is the number of distinct pairs of electrons with parallel spins. The crystal field potential here is the energy difference between the $t_{2 g}$ and the $e_{1 g}$ orbitals. 


\subsection{Introduction}

\subsubsection{Ligand Field Model}

As mentioned before, in the crystal field model, ligands are treated only as point charges by creating an electrostatic potential. Therefore, it ignores the nature of the ligands and hybridization effects completely. To take the crystal field model one step further, the bonding and covalency among the ligands and the metal are explicitly considered in the so-called "ligand field model" where the charge transfer among the atoms is allowed. Ligand field theory includes the molecular orbital theory concepts and symmetry concerns and allows bonding, anti-bonding and non-bonding hybridizations among the atomic orbitals.

One approach to the ligand field model is to include the central atom and the nearest neighbour ligands interacting through the configuration interaction mixing. The set of atoms included in the model is called a cluster and the arrangement of the electrons among the atoms is known as a configuration. This ligand field model is also known as the configuration interaction cluster model (or cluster calculation for short) where the wave-functions are expressed as a linear combination of different possible configurations.

Typically within cluster calculations, a many-electron basis is defined on which a Hamiltonian matrix is constructed. The Hamiltonian is then diagonalized, and the ground state energy and wave-functions are found using various algorithms such as the Lancsoz routine [97]. By having the wave-functions, the expectation value of different operators such as spin, angular momentum, number operators, etc. can be found. For spectroscopy simulations, a final state basis and the final state Hamiltonian also have to be defined. Then the spectra can be calculated by using the Greens function method:

$$
G(\omega)=\left\langle\psi_{i}\left|T^{\dagger} \frac{1}{\omega-H+i \frac{\Gamma}{2}} T\right| \psi_{i}\right\rangle,
$$

where $T$ is the transition operator. For an absorption process the transition operator would be a product of an annihilation and a creation operator, and for photoemission, it is only an annihilation operator [98, 99]. The relation between the photoemission transition probability and a Green function was given in section 4.1.2.

\section{Covalency}

Covalency is a type of interaction among different atoms of a material. It arises from sharing electrons among atomic orbitals of the atoms. Depending 


\subsection{Introduction}

on the symmetry of the atomic orbitals and their energies, different types of covalency or hybridization can occur. These interactions can be described as the overlap of the atomic orbitals or $\left\langle\psi_{A}|H| \psi_{B}\right\rangle$ where $\psi_{A}$ and $\psi_{B}$ are the wave-functions (orbitals) of the atom $A$ and atom $B$, respectively. Depending on the phases of the atomic orbitals and their symmetries these overlaps or hybridizations can be "constructive (anti-bonding)" or "destructive (bonding)".

In an anti-bonding interaction, for a simple diatomic molecule, the resulting molecular orbitals (MO) has a node between the two atoms. It means there is a lower electron density between them. An anti-bonding interaction increases the energy relative to the separated atoms, and a destructive overlap weakens the bond between them. On the other hand, when the atomic orbitals are added in phase, the overlap is constructive, and the electrons density is higher at the middle. This has a lower energy and is more stable than the two separate atoms. It is called a bonding interaction. When there is incompatible symmetry between the atomic orbitals, no interaction will happen. The resulting molecular orbitals are known as non-bonding orbitals. Non-bonding orbitals have the same energy as the separated atoms.

The resulting molecular orbitals are often labeled with $\sigma$ and $\pi$ symmetries. If the bonding $\mathrm{MO}$ is symmetrical with respect to the internuclear axis (axis joining the two nuclear centers), it is called a $\sigma$ orbital. An antibonding $\mathrm{MO}$ orbital with a $\sigma$ symmetry is called a $\sigma^{\star}$ orbital. If the bonding orbital is asymmetrical with respect to the internuclear axis, it is called $\pi$ orbital. A $\pi$ orbital finds an opposite phase by rotation about the internuclear axis. The anti-bonding $\pi$ orbital is called $\pi^{\star}$ orbital. $\pi$ bonds are usually weaker than $\sigma$ bonds. $\sigma$ and $\pi$ bonding and anti-bonding orbitals are illustrated in Fig. 5.3 for $p$ orbitals.

\section{Coulomb and exchange interactions}

In configuration interaction ligand field theory, there can be multiple possible distributions of electrons ,i.e., configurations or multiplets. These multiplets each can be at a different energy level due to their different electronelectron interaction energies. In Hartree-Fock type formalism these manybody electron-electron Coulomb interactions can be expressed in terms of one- and two-electron integral operators. This is shown below skipping the detailed mathematical derivation [87, 92]. 


\subsection{Introduction}

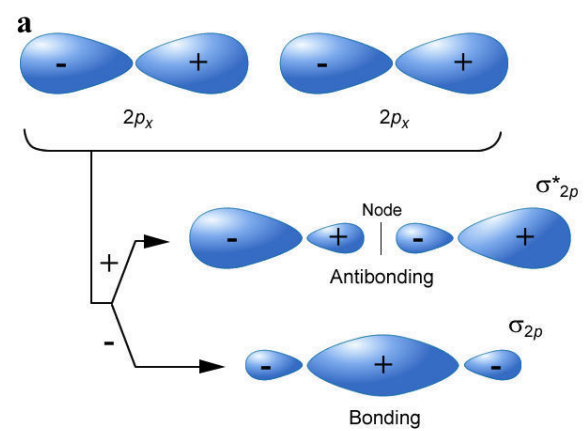

@NCSSM 2003

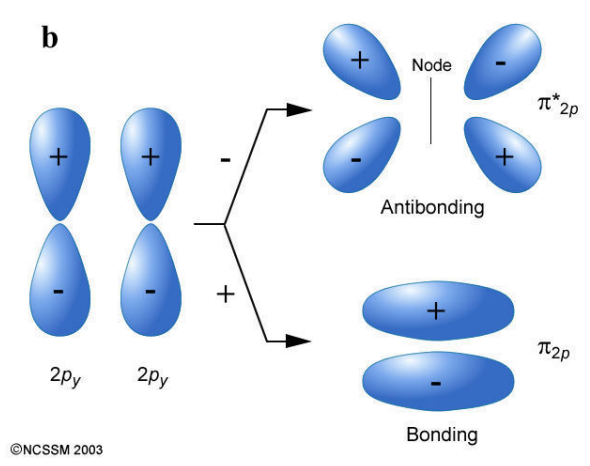

$\cos \operatorname{css} 2003$

Figure 5.3: A schematic representation of a: $\sigma$ and b: $\pi$ bonding and antibonding molecular orbitals of $p$ orbitals. The figure is taken from Ref. [19] and has been modified slightly by adding the signs.

$$
\begin{gathered}
E_{e-e}=\left\langle\Psi\left(x_{1}, \ldots, x_{N}\right)\left|H_{e-e}\right| \Psi\left(x_{1}, \ldots, x_{N}\right)\right\rangle \\
H_{e-e}=\sum_{i, j>i}^{N} \frac{1}{r_{i j}} \\
\Psi\left(x_{1}, \ldots, x_{N}\right) \equiv\left|\psi_{1}\left(\overrightarrow{x_{1}}\right) \psi_{2}\left(\overrightarrow{x_{2}}\right) \ldots \psi_{N}\left(\overrightarrow{x_{N}}\right)\right| \\
E_{e-e}=\frac{1}{2}\left[\sum_{m \neq n}^{N} \int \psi_{n}^{\star}\left(\overrightarrow{x_{1}}\right) \psi_{n}\left(\overrightarrow{x_{1}}\right) \frac{1}{r_{12}} \psi_{m}^{\star}\left(\overrightarrow{x_{2}}\right) \psi_{m}\left(\overrightarrow{x_{2}}\right) d x_{1} d x_{2}(5.1)\right. \\
\left.-\int \psi_{n}^{\star}\left(\overrightarrow{x_{1}}\right) \psi_{m}\left(\overrightarrow{x_{1}}\right) \frac{1}{r_{12}} \psi_{m}^{\star}\left(\overrightarrow{x_{2}}\right) \psi_{n}\left(\overrightarrow{x_{2}}\right) d x_{1} d x_{2}\right]=\langle n n \mid m m\rangle-\langle n m \mid m n\rangle
\end{gathered}
$$

In Eq. 5.10, the first and the second integrals are generally referred to as the Slater integral $\left(F^{k}\right)$ and the exchange integral $\left(G^{k}\right)$, respectively. The $\psi_{n}^{\star}\left(\overrightarrow{x_{1}}\right) \psi_{n}\left(\overrightarrow{x_{1}}\right)$ and the $\psi_{m}^{\star}\left(\overrightarrow{x_{2}}\right) \psi_{m}\left(\overrightarrow{x_{2}}\right)$ terms in the Slater integral are like the charge density distribution (or the probabilities) of electron 1 in orbital $n$ located at $x_{1}$ and electron 2 in orbital $m$ located at $x_{2}$, respectively. Therefore this term is similar to the classical Coulomb interaction energy. However, the exchange term does not have a classical counterpart and is rooted in the "Pauli exclusion principle". These two integrals can be expressed as one single integral in the following form where $P^{\prime} s$ are the Legendre polynomials, $r_{<}=\min \left(r_{1}, r_{2}\right)$, and $r_{>}=\max \left(r_{1}, r_{2}\right)$. 


$$
R^{k}(i j, k l)=\iint \frac{2 r_{<}^{k}}{r_{>}^{k+1}} P_{i}^{\star}\left(r_{1}\right) P_{j}^{\star}\left(r_{2}\right) P_{k}\left(r_{1}\right) P_{l}\left(r_{2}\right) d r_{1} d r_{2}
$$

where the Slater integral $F^{k}(i, j)=R^{k}(i j, i j)$, and the exchange integral $G^{k}(i, j)=R^{k}(i j, j i) . F^{0}$ and $F^{2}$ integrals are often known as the monopole and dipole Slater or Coulomb integrals, respectively.

\subsection{Computations}

In this work, we used the exact diagonalization cluster calculation method to investigate the electronic structure of $\mathrm{BaBiO}_{3}$. These calculations were done in quanty-"a quantum many-body script language". The code is developed by Maurits W. Haverkort and coworkers [100, 101] in the Lua programming language. It has several pre-defined operators in second quantization that allows the user to focus on the model and its physical aspects without worrying too much about the mathematics. In this section, we explain the model and present its results $[1]$.

\subsubsection{Cluster}

In our model, the cluster is an octahedron of six oxygens at the corners and a bismuth atom at the centre. There are three $p\left(p_{x}, p_{y}\right.$, and $\left.p_{z}\right)$ orbitals and a core $1 s$ orbital for each oxygen, and a $6 s$ orbital for the bismuth. All the oxygen core $1 s$ orbitals are full, and a hole will be created on one through the XPS process for the XPS spectra simulation part. There are 36 electrons in total in the oxygen $2 p$ and bismuth $6 s$ orbitals ,i.e., two holes in the octahedron. The octahedron is depicted in Fig. 5.4.

\subsubsection{Model Hamiltonian}

Our model Hamiltonian includes the following terms:

$$
\begin{array}{r}
H^{\text {total }}=H_{O_{p}}^{\text {hopping }}+H_{O_{p}}^{U}+H_{O_{p}}^{F^{2}}+H_{O_{s p}}^{Q}+H_{O_{s p}}^{G^{1}} \\
+H_{B i_{6 s}-O_{A_{1 g}}^{\text {hopping }}+H^{C F}+H^{\text {onsites }}}
\end{array}
$$

Each term will be explained below. 


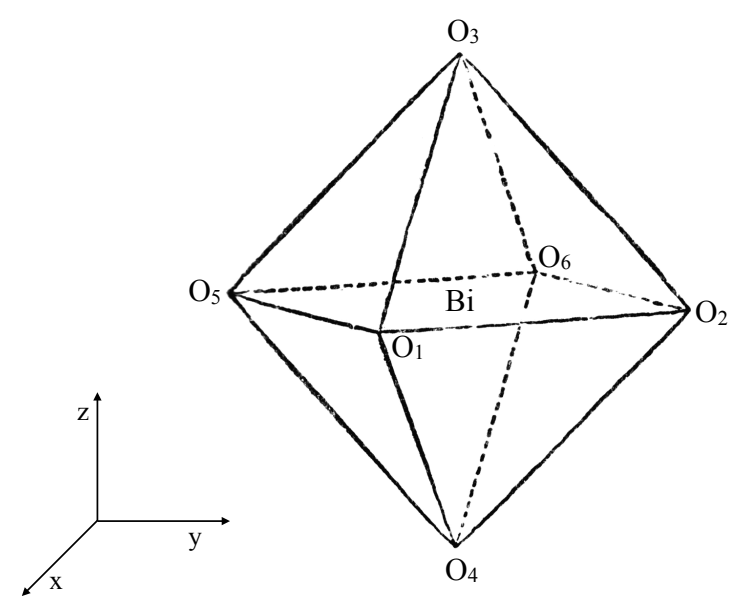

Figure 5.4: The model single cluster of $\mathrm{BaBiO}_{3}$ with six oxygens at the corners and a bismuth atom at the center.

\section{Hybridization terms: $H_{O_{p}}^{\text {hopping }}$ and $H_{B i_{6 s}-O_{A_{1 g}}}^{\text {hopping }}$}

In this model, we allow hybridization among the oxygens $p$ orbitals $\left(H_{O_{p}}^{\text {hopping }}\right)$, and also hopping between the bismuth and the oxygens $\left(H_{B i_{6 s}-O_{A_{1 g}}}^{\text {hopping }}\right)$.

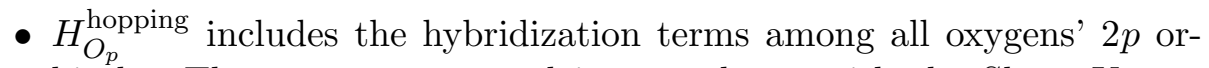
bitals. These are constructed in accordance with the Slater-Koster hopping matrix elements for an octahedral complex in terms of the $t_{p p \sigma}$ and $t_{p p \pi}$ hopping integrals[102].

$$
\begin{array}{r}
t_{p_{x}, p_{x}}=l^{2} t_{p p \sigma}+\left(1-l^{2}\right) t_{p p \pi} \\
t_{p_{x}, p_{y}}=l m t_{p p \sigma}-\operatorname{lm} t_{p p \pi} \\
t_{p_{x}, p_{z}}=l n t_{p p \sigma}-\ln t_{p p \pi}
\end{array}
$$

where $l, m$, and $n$ are the direction cosines to the neighbouring oxygens. The hopping energies are expressed in terms of $t_{p p \sigma}$ and $t_{p p \pi}$ energies. These energies are taken as $t_{p p \sigma}=0.63 \mathrm{eV}$ and $t_{p p \pi}=-0.04 \mathrm{eV}$ from tight-binding model parameterization of the band-structure 103]. Ref. [4] finds the very similar values of $t_{p p \sigma}=0.64 \mathrm{eV}$ and $t_{p p \pi}=-0.03$ $\mathrm{eV}$ for the sister compound $\mathrm{SrBiO}_{3}$. 


\subsection{Computations}

- $H_{B i_{6 s}-O_{A_{1 g}}^{\text {hopping }}}$ is the hybridization term between the bismuth $6 s$ orbital at the centre of the octahedron and the $A_{1 g}$ combination of the six $\mathrm{O}$ $2 p$ orbitals around. This combination is the only allowed symmetry combination with which an $s$ orbital can hybridize. Note that as shown below the effective Bi-O hopping energy is equal to $\sqrt{6} t_{s p \sigma}$ where $t_{s p \sigma}$ is the single bond hopping integral as schematically shown in Fig. 5.5-a:

$$
\begin{array}{r}
\mid \psi_{A_{1 g}}>=\frac{1}{\sqrt{6}}\left(\left|\psi_{2 p_{1}}>+\right| \psi_{2 p_{2}}>+\mid \psi_{2 p_{3}}>\right. \\
\left.+\left|\psi_{2 p_{4}}>+\right| \psi_{2 p_{5}}>+\mid \psi_{2 p_{6}}>\right) \\
t_{O_{p}-B i_{6 s}}=<\psi_{A_{1 g}}|H| \psi_{6 s}>=\frac{6}{\sqrt{6}}<\psi_{2 p_{1}}|H| \psi_{6 s}> \\
=\sqrt{6} t_{s p \sigma} .
\end{array}
$$

The same tight-binding model parameterization of the band-structure 103 . gives $t_{s p \sigma}=2.1 \mathrm{eV}$ for $\mathrm{BaBiO}_{3}$ structure. Ref. [4] finds $t_{s p \sigma}=2.3 \mathrm{eV}$ for $\mathrm{SrBiO}_{3}$. 


\subsection{Computations}

a

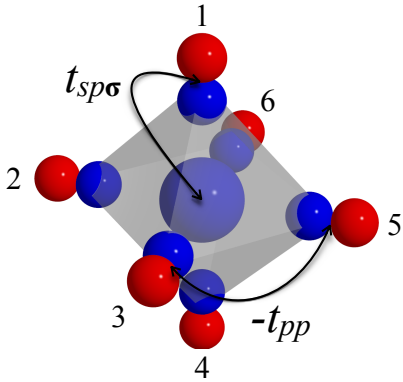

C

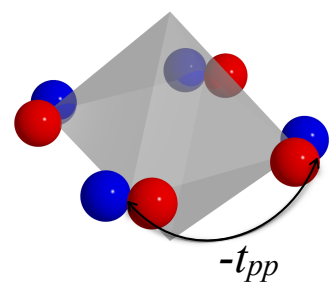

b

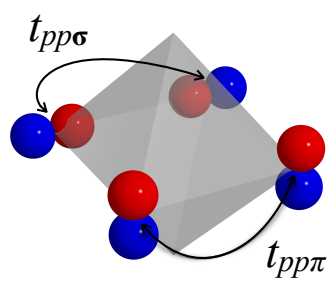

d

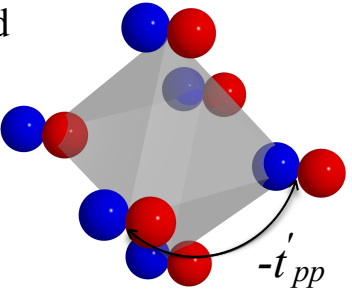

Figure 5.5: A schematic presentation of the involved hybridization terms in our model Hamiltonian. There are bismuth $s$ orbital at the centre and oxygen $p$ orbitals at the corners of the octahedron. a: $t_{O_{p}-B i_{6 s}}=\sqrt{6} t_{s p \sigma}$. b: $\sigma$ and $\pi$ oxygen $p$ hopping terms. c (and a): $t_{p p}=1 / 2\left(t_{p p \sigma}-t_{p p \pi}\right)$. d: $t_{p p}^{\prime}=1 / 2\left(t_{p p \sigma}+t_{p p \pi}\right)[1]$.

\section{Coulomb and Exchange Terms $: H_{O_{p}}^{U}, H_{O_{p}}^{F^{2}}, H_{O_{s p}}^{Q}$, and $H_{O_{s p}}^{G^{1}}$}

- $H_{O_{p}}^{U}$ : The monopole on-site Coulomb interaction between the electrons on the oxygen $p$ 's.

- $H_{O_{p}}^{F^{2}}$ : The higher multipole on-site Hund's rule interaction between the electrons on the oxygen $p$ 's.

- $H_{O_{s p}}^{Q}$ : The monopole Coulomb interaction between the $\mathrm{O} 1 s$ core hole and the $\mathrm{O}$ valence $2 p$ electrons.

- $H_{O_{s p}}^{G^{1}}$ : The exchange interaction between the $\mathrm{O} 1 s$ core hole and the $\mathrm{O}$ valence $2 p$ electrons.

Note that the Coulomb interaction between the two $s$ electrons on $\mathrm{Bi}$ are neglected in our model Hamiltonian because of the very large radial extend of the $6 s$ orbital with its 5 radial nodes. 
Crystal Field Energy: $H^{C F}$

Due to the crystal field potential produced by the bismuth's electrons, electrons in the $p$ orbitals pointing towards the cation $\left(p_{\sigma}\right)$ have a higher energy than the electrons in the orbitals perpendicular to it $\left(p_{\pi}\right)$ 104. This is taken into account in the Hamiltonian with the term: $\epsilon_{\sigma}-\epsilon_{\pi}=E_{C F}$.

\section{On-site Energies: $H^{\text {onsites }}$}

The on-site energies for the oxygen $1 s, 2 p$ and bismuth $6 s$ orbitals are expressed in terms of the charge transfer energy $(\Delta)$, monopole Coulomb repulsion $U$, and the core-hole potential $Q$ as shown below.

We define the charge transfer energy as the energy difference between the following two configurations.

$$
\begin{aligned}
& \left.E\left(\left|\psi_{O_{1 s}}^{12}>,\right| \psi_{B i_{6 s}}^{2}\right\rangle, \mid \psi_{O_{2 p}}^{34}>\right)=0 \\
& \left.E\left(\left|\psi_{O_{1 s}}^{12}>,\right| \psi_{B i_{6 s}}^{1}\right\rangle, \mid \psi_{O_{2 p}}^{35}>\right)=\Delta .
\end{aligned}
$$

where the superscripts are the number of electrons i.e $\mid \psi_{O_{2 p}}^{34}>$ means there are 34 electrons (or 2 holes) in O $2 p$ orbitals. According to DFT this material is a negative charge transfer material with $\Delta=-4 \mathrm{eV}[4]$.

The energies of the above configurations can be written in terms of $\Delta$, $U$ and $Q$ to find the on-site energies:

$$
\begin{gathered}
12 \epsilon_{1 s}+2 \epsilon_{6 s}+34 \epsilon_{2 p}+\left[4 \times\left(\begin{array}{l}
6 \\
2
\end{array}\right)+2 \times\left(\begin{array}{l}
5 \\
2
\end{array}\right)\right] \times U+2 \times[4 \times 6+2 \times 5] Q=\Delta \\
12 \epsilon_{1 s}+1 \epsilon_{6 s}+35 \epsilon_{2 p}+\left[5 \times\left(\begin{array}{l}
6 \\
2
\end{array}\right)+1 \times\left(\begin{array}{l}
5 \\
2
\end{array}\right)\right] \times U+2 \times[5 \times 6+1 \times 5] Q=0
\end{gathered}
$$

Now we set the $\epsilon_{1 s} \equiv 0$ and solve for the other on-site energies to yield:

$$
\begin{array}{r}
e_{1 s} \equiv 0 \\
e_{2 p}=\frac{-\Delta-45 U-36 Q}{18}, \\
e_{6 s}=\frac{17 \Delta+45 U}{18} .
\end{array}
$$

The values of the the core-hole potential $Q$, monopole Coulomb energy $U$, and the crystal field potential $E_{C F}$ are taken as $3.6 \mathrm{eV}, 3 \mathrm{eV}$ and $1 \mathrm{eV}$, 
respectively. These values do not deviate from the well-established ranges over the past decades $[12,85,104-106$. The dipole Coulomb and exchange terms ( $F^{2}$ and $G^{1}$, respectively) are also taken from atomic oxygen calculated values as $G_{s p}^{1}=3.04 \mathrm{eV}$ and $F_{p p}^{2}=7.25 \mathrm{eV}$.

\subsection{Ground-State Spin Calculation}

We begin the calculation by first examining the spin state of the ground state for various values of the crystal field energy $\left(E_{C F}\right)$, and the oxygenbismuth hybridization energy $\left(t_{s p \sigma}\right)$. We know $\mathrm{BaBiO}_{3}$ is a diamagnetic material with the total spin of zero in its ground state [4, 7]. On the other hand, tight-binding calculation for an octahedron of only six oxygens at the corners with two holes gives a triplet ground state with wave function of mainly $\left(e_{g}\right)^{2}$ configuration when $U>0[104]$. However, when the bismuth cation is added to the system and allowed to hybridize with the oxygens, the ground state has to change to the expected singlet state with an $A_{1 g}$ symmetry where the two holes in the ground state have to be antiparallel with the total spin of zero (low-spin) due to the Pauli exclusion principle.

We examine the above statement by finding the total spin of the ground state for various values of the crystal field energy $\left(E_{C F}\right)$, and the oxygenbismuth hybridization energy $\left(t_{s p \sigma}\right)$. As shown in Fig. 5.6, starting from $E_{C F}=0$, and $t_{s p \sigma}=0$, first we need to turn on the $E_{C F}$, so that for $t_{s p \sigma}=0$ we get the correct triplet ground state. When $t_{s p \sigma}=0$ the system is basically without the bismuth cation and consists of only the six oxygens. Then we have to increase the $t_{s p \sigma}$ energy so that we achieve the expected low spin ground state when the bismuth is present. These minimum or threshold $E_{C F}$ and $t_{s p \sigma}$ energies are small enough that for any reasonable value we achieve the correct ground state in our calculation. Remember that our expected values of $t_{s p \sigma}$ and $E_{C F}$ are respectively $2.1 \mathrm{eV}$ and $1 \mathrm{eV}$ from previous tight-binding calculations [103, 104]. Therefore, the ground state will always stay in the low spin blue region above this graph as expected. 


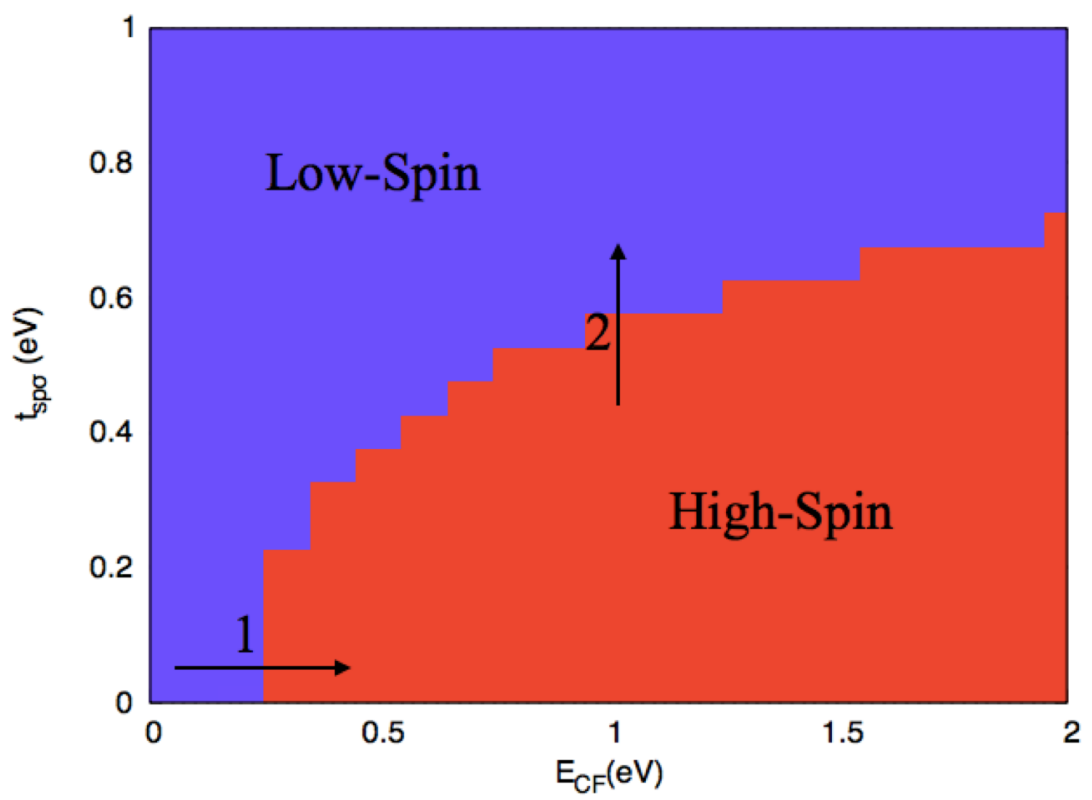

Figure 5.6: The total spin of the ground state as a function of crystal field energy $\left(E_{C F}\right)$ and oxygen-bismuth hybridization energy $\left(t_{s p \sigma}\right)$ for $U=3$ $\mathrm{eV}$. The first arrow indicates that we need to turn on the $E_{C F}$, so that for $t_{s p \sigma}=0$ we get the correct triplet ground state for a bismuthless octahedron with two holes. The second arrow shows we have to increase the $t_{s p \sigma}$ energy so that we achieve the expected low spin ground state when the bismuth is present.

\subsection{Hole Distribution Calculation}

Now we continue the calculation by finding the hole distribution between the ligand oxygens and the bismuth cation in the octahedron. As described in chapter 1 the insulating mechanism of $\mathrm{BaBiO}_{3}$ is often explained in terms of charge disproportion of $\mathrm{Bi}^{4+}+\mathrm{Bi}^{4+}$ into $\mathrm{Bi}^{3+}$ and $\mathrm{Bi}^{5+}$. In this picture, it is assumed the oxygen anions are full, and holes reside on the bismuth cation. However, many experimental studies [28-30] as well as band structure calculations [4, 26] find relatively small charge differences between the bismuth sites.

We also showed in chapter 3 that core-level spectroscopy measurements such as XAS give evidence for having holes on the oxygens whereas the XPS 
result in chapter 4 might not support that. Here, we address this question by calculating the average number of holes on the oxygen anions versus the bismuth cation for a wide range of $\Delta$ and $t_{s p \sigma}$. As shown in Fig. 5.7, for a wide range of these energies, holes indeed mainly reside on the ligand oxygens. 


\subsection{Hole Distribution Calculation}

a

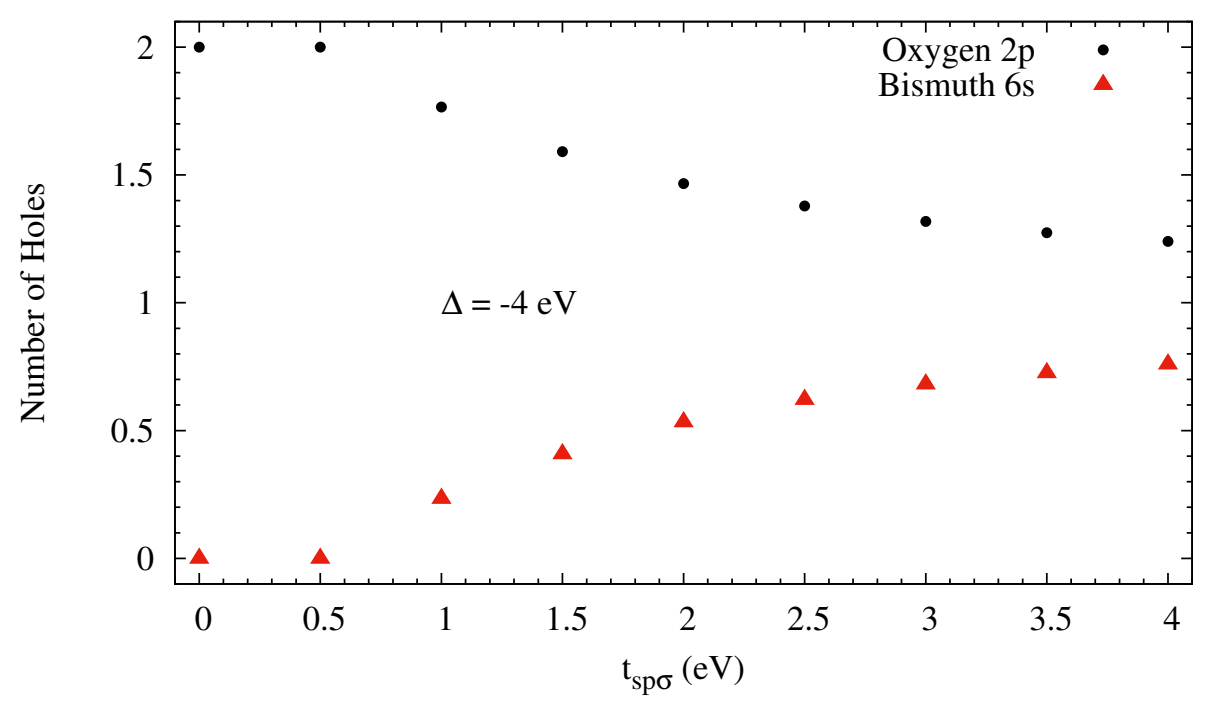

b

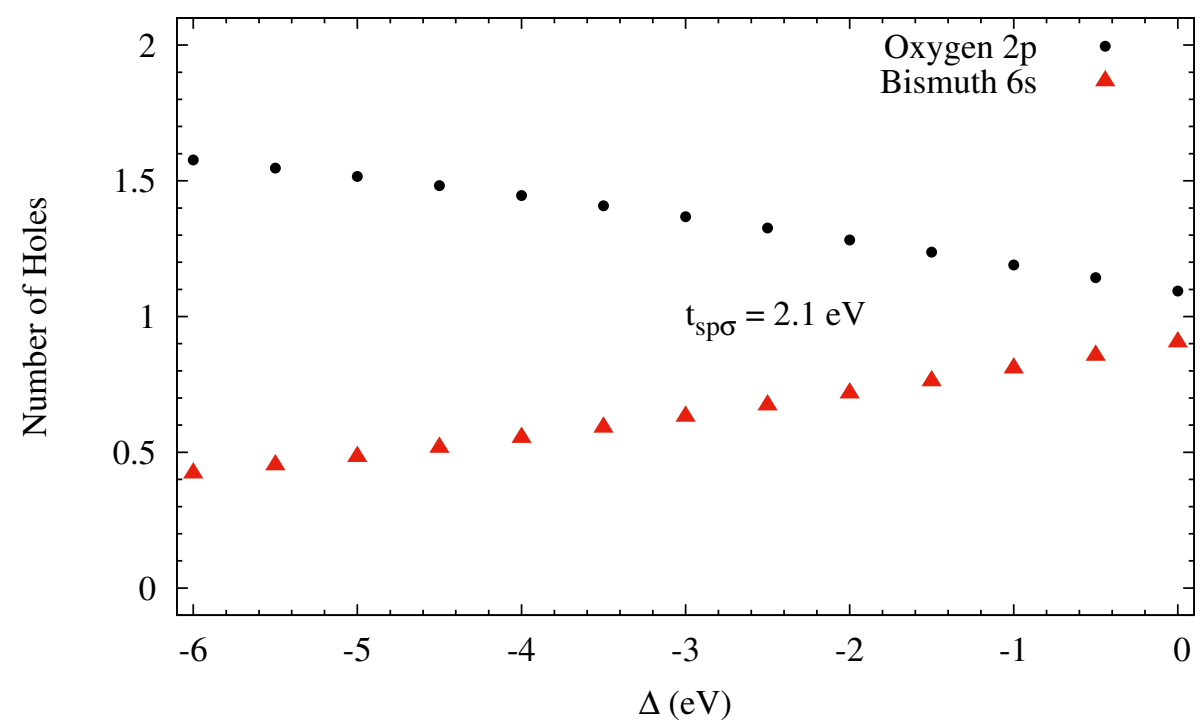

Figure 5.7: The average number of holes as a function of $a$ : $\mathrm{Bi} 6 s / \mathrm{O} p$ hybridization energy $\left(t_{s p \sigma}\right)$ for $\Delta=-4 \mathrm{eV}$. and $b$ : the charge transfer energy $\Delta$ for $t_{s p \sigma}=2.1 \mathrm{eV}$. For a large range of parameters, holes reside primarily on the ligand oxygens. 


\subsection{XPS calculation}

In the previous chapter, we presented the oxygen $1 s$ x-ray photoemission measurements on $\mathrm{BaBiO}_{3}$ single crystal. We showed how the spectrum loses most of its structure through the progression of the surface cleaning procedure and becomes almost structureless but remains slightly asymmetric. We discussed how one would expect a different result due to the discussed high hole density on the oxygens in chapter 3 . In the previous section, we calculated that indeed there is a high hole density on the ligand oxygens. Therefore, the question still remains regarding the shape of the oxygen $1 \mathrm{~s}$ XPS structure. To address this problem, in this section we calculate the oxygen $1 s$ XPS spectra of $\mathrm{BaBiO}_{3}$ for varying parameters.

We begin by setting the $\mathrm{O}-\mathrm{O}$ and $\mathrm{O}-\mathrm{Bi}$ hybridization to zero. We then calculate the O $1 s$ XPS for different core hole potentials $(Q)$ while preserving the $\frac{Q}{U}=1.2$ ratio [107]. Because the O-Bi hybridization is zero and we have a negative charge transfer energy, the two holes reside entirely on the oxygen sites. Further, since we have no O-O hybridization, we have a set of degenerate ground states where the two holes are distributed among the six oxygen sites (though with no double occupancy when there is a finite Coulomb interaction $U$ ). Averaging over the degenerate states, each oxygen site has a $\frac{1}{3}$ probability of hosting one of the two holes.

As can be seen from Fig. 5.8, for the case of $Q=0$ only a single peak is present in the XPS. The energy of this peak is $0 \mathrm{eV}$, as we set the onsite energy of the core $1 s$ level to zero for simplicity here. For a finite $Q$, two peaks are present in the XPS. The more intense peak originates from the case where the core hole is created on a site where there is no valence $2 p$ hole. The weaker peak at $+Q$ higher in energy originates from the case where the core hole is created on a site having a valence $2 p$ hole. The $2: 1$ intensity ratio between the two peaks is to be expected: there is a $\frac{1}{3}$ probability that a valence hole is on the core hole site and a $\frac{2}{3}$ probability that there is no valence hole. This example shows how one might expect to see the effect of the self-doped oxygen holes in the oxygen XPS through the appearance of a satellite peak.

Now to examine the effect of the bismuth atom at the centre and oxygens' $p-p$ hybridization, we turn on the $p-p$ hopping terms and then increase the Bi-O hopping, $t_{s p \sigma}$, from 0 to $2 \mathrm{eV}$. As shown in Fig. 5.9, we get a relatively strong satellite structure with turning on the hybridization among the $p$ orbitals due to the holes distributed among the ligand oxygens. However, when we allow the hybridization between the $\mathrm{Bi} 6 s$ and the oxygens, their intensities are suppressed dramatically. This is due to the strong dilution 


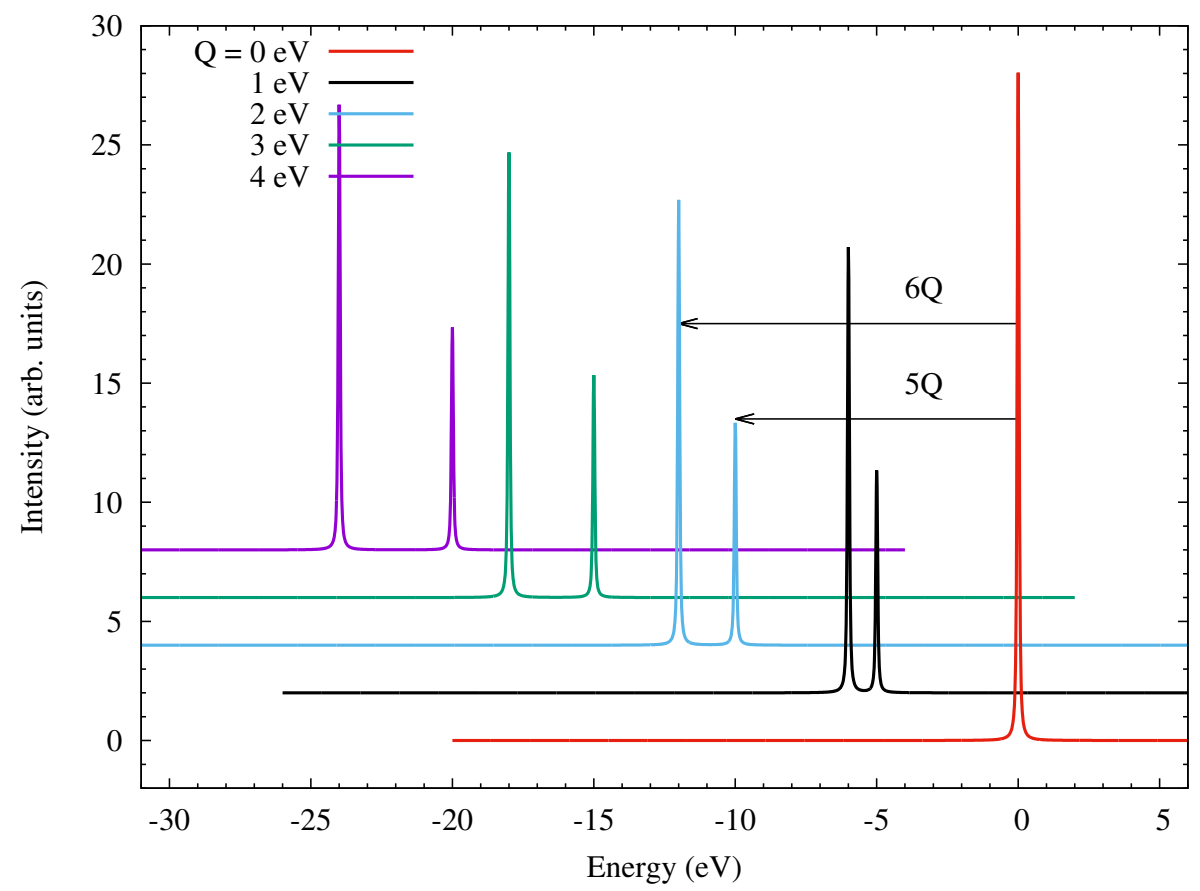

Figure 5.8: Calculated oxygen $1 s$ XPS spectra with all the hybridization terms set to zero for different core-hole potential $(Q)$ values. The curves are shifted along the "Intensity" axis. 


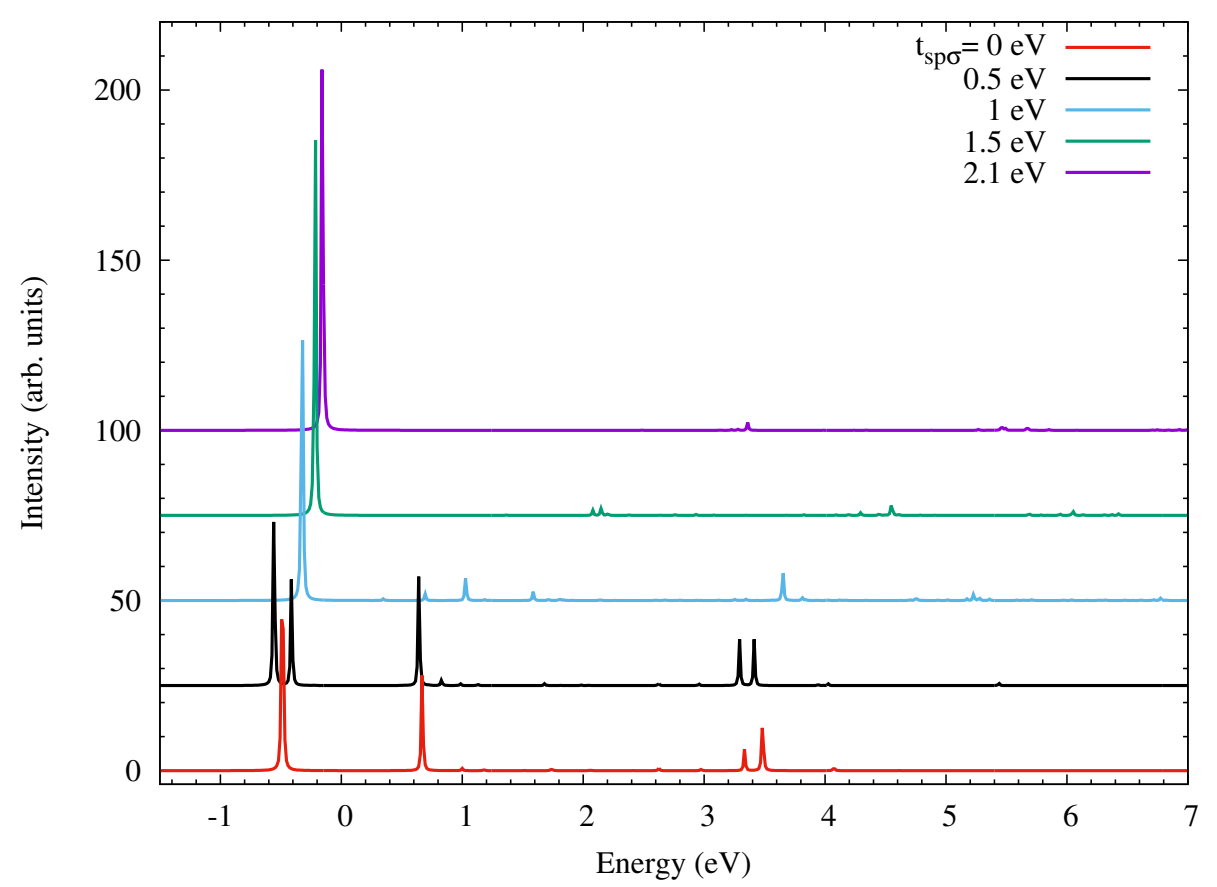

Figure 5.9: Calculated oxygen $1 s$ XPS spectra for different Bi $6 s / \mathrm{O} p$ hybridization energies $\left(t_{s p \sigma}\right)$, for $t_{p p \pi}=-0.04 \mathrm{eV}, t_{p p \sigma}=0.63 \mathrm{eV}, U=3 \mathrm{eV}$, $Q=3.6 \mathrm{eV}$, and $E_{C F}=1 \mathrm{eV}$. Multiplets lose their intensities significantly with turning on the bismuth-oxygen hybridization. The curves are shifted along the "Intensity" axis.

of the influence of the oxygen $1 s$ core hole which has negligible interaction with the Bi $6 s$ hole.

This result explains the relatively sharp but slightly asymmetric $\mathrm{O} 1 \mathrm{~s}$ peak of the XPS experiment even in the presence of a substantial oxygen $2 p$ hole concentration. This analysis provides direct evidence for the dominant influence of the oxygen $2 p$ - bismuth $6 s$ hybridization. This study provides further insight into the importance of self-doped ligand oxygen $2 p$ states in this high $T_{c}$ family of oxides. It shows that the O $2 p$ states should play an essential role in the description of what happens with the hole or electron doping of the bismuthates and therefore, in the description of the origin of superconductivity in the doped materials. 


\subsection{Conclusion}

\subsection{Conclusion}

We employed exact diagonalization cluster calculation on an octahedron of six oxygens around and a bismuth at the center with two holes in total. We found that for a wide range of charge transfer energies and $\mathrm{O} 2 p$-Bi $6 s$ hybridization energies, holes mainly reside on the ligand oxygens rather than the conventionally accepted purely ionic picture that assigns holes to the bismuth cation.

We also used this model, to simulate the oxygen $1 s$ XPS spectrum for varying values of core-hole potential and $\mathrm{O} 2 p$-Bi $6 s$ hybridization. From this analysis along with the XPS experimental results of chapter 4 , we conclude that the $\mathrm{O} 2 p$ - $\mathrm{Bi} 6 s$ hybridization energy is the dominant energy scale in this problem which determines the low energy scale electronic structure. 


\section{Chapter 6}

\section{Summary and Future Work}

\subsection{Summary}

In chapter 1 , we reviewed the main properties of the material, $\mathrm{BaBiO}_{3}$, including its general electronic and crystal structure and its superconducting phase. We also discussed the importance of studying the roles of oxygen anions in this material by comparing its properties to nickelates and cuprates.

In chapter 2, we presented our congruent melting technique to grow single crystals of $\mathrm{BaBiO}_{3}$ with sizes up to $2 \times 2 \times 1 \mathrm{~mm}^{3}$. We then characterized the grown crystals by XPS chemical composition measurement, XRD and resistivity measurements. They all confirmed the growth of high-quality single crystals. We showed that using a two-step solid state reaction before the melt growth and also using a wide crucible during the growth are crucial in order to get reasonably big single crystals

In chapter 3 , we used oxygen K-edge XAS and RXES to study the oxygens' bonding and antibonding $p$-bands close to the chemical potential. The result was then compared to the DFT(LDA) oxygen- $p$ projected density of states. These gave evidence for a high hole concentration in the oxygens' states in contrast to the generally assumed purely ionic chargedisproportionation picture where holes are believed to reside mainly on the bismuth cations.

In chapter 4, we proceeded with the investigation of the oxygens' roles in the electronic structure of the $\mathrm{BaBiO}_{3}$ using detailed oxygen $1 s$ XPS. We showed surface contamination can be a serious issue in interpreting the structure of the oxygen spectrum. We presented our cleaning routine which was successful in removing the carbon-containing compounds from the surface to achieve a reliable oxygen $1 s$ spectrum. We showed by removing the surface contamination, oxygen $1 s$ spectrum also loses much of its structure and its shoulder but remains slightly asymmetric. We were later able to explain this result by performing exact diagonalization cluster calculations in chapter 5 .

Finally, in chapter 5, we did exact diagonalization model calculation on an octahedron of six oxygens at the corners and a bismuth at the centre to 


\subsection{Future Works}

find the hole distribution between the oxygens and the bismuth, and also to simulate the oxygen $1 s$ XPS spectrum for different electronic energies. We demonstrated that for a wide range of charge transfer and hybridization energies, holes mainly reside on the ligand oxygens in agreement with the results from chapter 3 . The XPS calculation and its comparison with the experimental spectrum from chapter 4 also suggested that the $\mathrm{O} 2 p$-Bi $6 s$ hybridization energy has to be the dominant energy scale in this problem which determines the low energy scale electronic structure.

\subsection{Future Works}

\subsubsection{Further Characterization of the Single Crystals}

In chapter 2, we characterized the crystal structure of the grown $\mathrm{BaBiO}_{3}$ crystal by XRD and found its chemical composition by XPS and also performed temperature dependent electronic transport measurements. However, the crystals are still being characterized in more detail by measuring their magnetic susceptibility, the Young modulus of elasticity and also studying the surface by the Atomic Force Microscopy technique and careful optical photography.

\subsubsection{Electron Doped Material}

To this date, there has been no significant success in making an electrondoped $\mathrm{BaBiO}_{3}$ compound. Finding such a compound in a crystalline form is unlikely due to the ionic radii of the candidate dopants. Lanthanum is usually proposed to substitute the barium to add electrons to the system. However, considering the ionic radii of the involved ions, most probably the Lanthanum $3+$ will take the Bi $3+$ sites rather than the Ba 2+ sites [38]:

- Ionic radius of $\mathrm{Ba}^{2+}$ with the coordination number of $12: 1.61 \stackrel{\circ}{A}$

- Ionic radius of $\mathrm{Bi}^{3+}$ with the coordination number of $6: 1.03 \AA$

- Ionic radius of $\mathrm{La}^{3+}$ with the coordination number of $12: 1.36 \stackrel{\AA}{A}$

- Ionic radius of $\mathrm{La}^{3+}$ with the coordination number of $6: 1.032 \AA$

It can be seen that $\mathrm{La}^{3+}$ and $\mathrm{Bi}^{3+}$ with six coordination numbers have very close ionic radii, while the $\mathrm{La}^{3+}$ and $\mathrm{Ba}^{2+}$ with 12 coordination numbers have quite different ionic radii. Remember in the $\mathrm{ABO}_{3}$ perovskite structure, 
$\mathrm{A}$ and $\mathrm{B}$ atoms have coordination numbers of 12 and six, respectively. This problem exists for the other proposed dopants such as yttrium, too.

However, electron doping the $\mathrm{BaBiO}_{3}$ film might be possible by using an electron donor like caesium atoms placed on top of the surface. Of course, this would only create electron doping close to the surface but would be a way to get electrons into the system, regardless. It has also been suggested by our group that one could use an alternation of a polar material with $\mathrm{SrBiO}_{3}$ in heterostructure in which $\mathrm{SrBiO}_{3}$ would be electron or hole doped in order to compensate for the polar divergence of the potential in the polar materials [108]. Growing and studying such a martial, which can very well be superconducting, will be very interesting and also give valuable insights regarding these systems.

\subsubsection{Resonant Photoemission Measurements}

A detailed resonant x-ray photoemission measurement looking at bismuth $p \rightarrow s$ transition can be an effective way to gather further information on the amount of $s$ and $p$ character in the density of states of the material. In RPES, the photoemission signal is recorded while varying the photon energy across an x-ray absorption edge or a resonance energy where the incident photon excites an electron from the resonating core state into an empty state above the chemical potential. From the band structure calculation [4], we would expect to see about $50 \%$ bismuth $6 s$ character just below the Fermi energy, but also some at a much deeper energy.

The momentum resolved band structure of single crystal $\mathrm{BaBiO}_{3}$ can also be measured by Angular Resolved PES technique. This has been done on the thin film material [109] but yet to be performed on a single crystal.

\subsubsection{Pair Distribution Function Measurements}

In this thesis, we presented further evidence for the bond-disproportionated picture in the undoped $\mathrm{BaBiO}_{3}$ where there are compressed and expanded octahedra with two and zero holes in them. A careful study of Pair Distribution Function in the potassium substituted systems can be very informative in answering the question that whether those long-short bond fluctuations still exist in the hole-doped material and whether or not the bond lengths are varying over the same ranges as it is in the undoped material. This, in turn, helps to understand their superconducting mechanism and whether it is a bipolaron problem with a strong PDF or a BCS like situation. 


\section{Bibliography}

[1] Shadi Balandeh, Robert J. Green, Kateryna Foyevtsova, Shun Chi, Oleksandr Foyevtsov, Fengmiao Li, and George A. Sawatzky. Experimental and theoretical study of the electronic structure of singlecrystal $\mathrm{BaBiO}_{3}$. Phys. Rev. B, 96:165127, Oct 2017.

[2] D.E. Cox and A.W. Sleight. Crystal structure of $\mathrm{Ba}_{2} \mathrm{Bi}^{3+} \mathrm{Bi}^{5+} \mathrm{O}_{6}$. Solid State Communications, 19(10):969 - 973, 1976.

[3] John F. Watts and John Wolstenholme. An Introduction to Surface Analysis by XPS and AES. WILEY, 2005.

[4] Foyevtsova Kateryna, Khazraie Arash, Elfimov Ilya, and Sawatzky George A. Hybridization effects and bond disproportionation in the bismuth perovskites. Phys. Rev. B, 91:121114, 2015.

[5] George A. Sawatzky and Robert Green. The explicit role of anion states in high valence metal oxides. Lecture notes of the Autumn School on Correlated Electrons, Forschungszentrum Julich, 092016.

[6] J. Zaanen, G.A. Sawatzky, and J.W. Allen. Band gaps and electronic structure of transition-metal compounds. Phys. Rev. Lett. 55, 1985.

[7] A. W. Sleight. Bismuthates: $\mathrm{BaBiO}_{3}$ and related superconducting phases. Physica $C$, 514:152-165, 2015.

[8] Brian R. Pamplin, editor. Crystal Growth,, volume 16. Pergamon press, second edition, 1980.

[9] Ikuyo Shiozaki and Hiroyoshi Ishii. Crystal Growth and Electronic Structure of Metal Substituted $\mathrm{BaBiO}_{3}$. Japanese Journal of Applied Physics, 32(S3):686, 1993.

[10] A. Herrera-Gomez, M. Bravo-Sanchez, O. Ceballos-Sanchez, and M. O. Vazquez-Lepe. Practical methods for background subtraction in 
photoemission spectra. ECASIA special issue paper, 2014 John Wiley ES Sons, Ltd., 2014.

[11] Robert J. Green. Transition Metal Impurities in Semiconductors: Induced Magnetism and Band Gap Engineering. PhD thesis, University of Saskatchewan, 2013.

[12] F. M. F. de Groot and A. Kotani. Core Level Spectroscopy of Solids. CRC Press, Taylor \& Francis Group, 2008.

[13] http://reixs.lightsource.ca/about/rsxs/.

[14] http://www.lightsource.ca/beamlines.

[15] William F. Stickle John F. Moulder and et al. Handbook of X-ray Photoelectron Spectroscopy. Physical Electronics, Inc., 1995.

[16] Kai Siegbahn. ESCA [Electron Spectroscopy for Chemical Analysis]: Atomic, Molecular and Solid State Structure Studied by Means of Electron Spectroscopy. Almquist and Wiksells, 1967.

[17] Friedrich Reinert and Stefan Hfner. Photoemission spectroscopy - from early days to recent applications. 7:97, 042005.

[18] Kai Siegbahn; et al. ESCA applied to free molecules. Amsterdam ; London : North-Holland Publishing,, 1969.

[19] Teachers' Instructional Graphics Educational Resource. http://www.dlt.ncssm.edu/tiger/chem3.htm.

[20] R. Scholder, K. Ganter, H. Glaser, and G. Merz. Alkali and alkali earth bismuthates (v). Zeit. Anorg. Allgem. Chem. 319, 1963.

[21] A.W. Sleight, J.L. Gillson, and P.E. Bierstedt. High-temperature superconductivity in the $\mathrm{BaPb}_{1-x} \mathrm{Bi}_{x} \mathrm{O}_{3}$ systems. Solid State Communications, 17(1):27 - 28, 1975.

[22] R. J. Cava, B. Batlogg, J. J. Krajewski, R. Farrow, L. W. Rupp Jr, A. E. White, K. Short, W. F. Peck, and T. Kometani. Superconductivity near $30 \mathrm{~K}$ without copper: the $\mathrm{Ba}_{0.6} \mathrm{~K}_{0.4} \mathrm{BiO}_{3}$ perovskite. Nature, 332:-, 1988.

[23] J.G. Bednorz and K.A. Muller. Possible high Tc superconductivity in the Ba-La-Cu-O system. Z. Phys. B, 64, 1986. 
[24] J. F. Federici, B. I. Greene, E. H. Hartford, and E. S. Hellman. Optical characterization of excited states in $\mathrm{BaBiO}_{3}$. Phys. Rev. B, 42:923926, Jul 1990.

[25] G. Thornton and A. J. Jacobson. A neutron diffraction determination of the structures of $\mathrm{Ba}_{2} \mathrm{Sb}^{V} \mathrm{Bi}^{I} I I \mathrm{O}_{6}$ and $\mathrm{Ba}_{2} \mathrm{Bi}^{V} \mathrm{Bi}^{I} I I \mathrm{O}_{6}$. Acta Crystallographica Section B, 34(2):351-354, Feb 1978.

[26] L. F. Mattheiss and D. R. Hamann. Electronic structure of $\mathrm{BaPb}_{1-x} \mathrm{Bi}_{x} \mathrm{O}_{3}$. Phys. Rev. B, 28:4227-4241, Oct 1983.

[27] A.Yu. Ignatov. Relationship between the electronic and local structure in $\mathrm{BaPb}_{x} \mathrm{Bi}_{1-x} \mathrm{O}_{3}$ and $\mathrm{Ba}_{1-x} \mathrm{~K}_{x} \mathrm{BiO}_{3}$ perovskites. Nuclear Instruments and Methods in Physics Research Section A: Accelerators, Spectrometers, Detectors and Associated Equipment, 448(1):332 - 339, 2000.

[28] Z.-X. Shen, P. A. P. Lindberg, B. O. Wells, D. S. Dessau, A. Borg, I. Lindau, W. E. Spicer, W. P. Ellis, G. H. Kwei, K. C. Ott, J.-S. Kang, and J. W. Allen. Photoemission study of monoclinic $\mathrm{BaBiO}_{3}$. Phys. Rev. B, 40:6912-6918, Oct 1989.

[29] G. K. Wertheim, J. P. Remeika, and D. N. E. Buchanan. Electronic structure of $\mathrm{BaPb}_{1-x} \mathrm{Bi}_{x} \mathrm{O}_{3}$. Phys. Rev. B, 26:2120-2123, Aug 1982.

[30] M. S. Hegde, P. Barboux, C. C. Chang, J. M. Tarascon, T. Venkatesan, X. D. Wu, and A. Inam. Electronic structure of high- $T_{c} \mathrm{Ba}_{0.6} \mathrm{~K}_{0.4} \mathrm{BiO}_{3}$ by x-ray photoelectron spectroscopy. Phys. Rev. B, 39:4752-4755, Mar 1989.

[31] Winiarski A., Wbbeler G., and Scharfschwerdt C. et al. Photoemission studies of $\mathrm{BaPb}_{1 x} \mathrm{Bi}_{x} \mathrm{O}_{3}$ and $\mathrm{Ba}_{1 y} \mathrm{~K}_{y} \mathrm{BiO}_{3}$ crystals. Fresenius $J$ Anal Chem, 341, 1991.

[32] R. E. Peierls. Quantum Theory of Solids. Clarendon, Oxford, 1955.

[33] F. R. S. H. Frhlich. On the theory of superconductivity: the onedimensional case. The Royal Society, 1954.

[34] M. Qvarford, V. G. Nazin, A. A. Zakharov, M. N. Mikheeva, J. N. Andersen, M. K. J. Johansson, G. Chiaia, T. Rogelet, S. Söderholm, O. Tjernberg, H. Nylén, I. Lindau, R. Nyholm, U. O. Karlsson, S. N. Barilo, and S. V. Shiryaev. Photoemission and x-ray absorption study of superconducting and semiconducting $\mathrm{Ba}_{1-x} \mathrm{~K}_{x} \mathrm{BiO}_{3}$ single crystals. Phys. Rev. B, 54:6700-6707, Sep 1996. 
[35] T. Mizokawa, D. I. Khomskii, and G. A. Sawatzky. Spin and charge ordering in self-doped mott insulators. Phys. Rev. B, 61:11263-11266, May 2000.

[36] Johnston S., Mukherjee A., Elfimov I., Berciu M., and Sawatzky G.A. Charge disproportionation without charge transfer in the rare-earthelement nickelates as a possible mechanism for the metal-insulator transition. Phys. Rev. Lett. 11, 2014.

[37] D. E. Cox and A. W. Sleight. Mixed-valent $\mathrm{Ba}_{2} \mathrm{Bi}^{3+} \mathrm{Bi}^{5+} \mathrm{O}_{6}$ : structure and properties vs temperature. Acta Cryst. B35, 1-10, 1979.

[38] R. D. Shannon. Central Research and Experimental Station Development Department. Revised effective ionic radii and systematic studies of interatomic distances in halides and chalcogenides". Acta Crystallographica. A32, Pages 751-767., 1976.

[39] Jiangang He, Cesare Franchini, and James M. Rondinelli. Ferroelectric oxides with strong visible-light absorption from charge ordering. Chemistry of Materials, 29(6):2445-2451, 2017.

[40] B. Batlogg, R. J. Cava, L. W. Rupp, A. M. Mujsce, J. J. Krajewski, J. P. Remeika, W. F. Peck, A. S. Cooper, and G. P. Espinosa. Density of States and Isotope Effect in $\mathrm{BiO}$ Superconductors: Evidence for Nonphonon Mechanism. Phys. Rev. Lett., 61:1670-1673, Oct 1988.

[41] D.T. Marx, P.G. Radaelli, J.D. Jorgensen, R.L. Hitterman, D.G. Hinks, S. Pei, and B. Dabrowski. Metastable behavior of the superconducting phase in the babi1xpbxo3 system. Phys. Rev. B, 46, 1992.

[42] D. Nicoletti, E. Casandruc, D. Fu, P. Giraldo-Gallo, I. R. Fisher, and A. Cavalleri. Anomalous relaxation kinetics and charge-density-wave correlations in underdoped $\mathrm{BaPb}_{1-x} \mathrm{Bi}_{x} \mathrm{O}_{3}$. Proceedings of the $\mathrm{Na}$ tional Academy of Sciences, 114(34):9020-9025, 2017.

[43] R. Comin, A. Frano, M. M. Yee, Y. Yoshida, H. Eisaki, E. Schierle, E. Weschke, R. Sutarto, F. He, A. Soumyanarayanan, Yang He, M. Le Tacon, I. S. Elfimov, Jennifer E. Hoffman, G. A. Sawatzky, B. Keimer, and A. Damascelli. Charge Order Driven by Fermi-Arc Instability in $\mathrm{Bi}_{2} \mathrm{Sr}_{2-x} L a_{x} \mathrm{CuO}_{6+}$. Science, 343(6169):390-392, 2014. 
[44] Eduardo H. da Silva Neto, Pegor Aynajian, Alex Frano, Riccardo Comin, Enrico Schierle, Eugen Weschke, András Gyenis, Jinsheng Wen, John Schneeloch, Zhijun Xu, Shimpei Ono, Genda Gu, Mathieu Le Tacon, and Ali Yazdani. Ubiquitous interplay between charge ordering and high-temperature superconductivity in cuprates. Science, 343(6169):393-396, 2014.

[45] M. Braden, W. Reichardt, E. Elkaim, J. P. Lauriat, S. Shiryaev, and S. N. Barilo. Structural distortion in superconducting $\mathrm{Ba}_{1-x} \mathrm{~K}_{x} \mathrm{BiO}_{3}$. Phys. Rev. B, 62:6708-6715, Sep 2000.

[46] C. Bougerol-Chaillout, P. Bordet, S. Kazakov, J. Pshirkov, S.N. Putilin, E.V. Antipov, and M. Nunez-Regueiro. Structural studies of new superconducting bismuthates $(\mathrm{Sr}, \mathrm{K}) \mathrm{BiO}_{3}$. Physica C: Superconductivity, 341-348:1813 - 1816, 2000.

[47] Izumi Tomeno and Ken Ando. Superconducting properties of the $\mathrm{Ba}_{1-\mathrm{x}} \mathrm{Rb}_{\mathrm{x}} \mathrm{BiO}_{3}$ system. Phys. Rev. B, 40:2690-2693, Aug 1989.

[48] S. Kondoh, M. Sera, Y. Ando, and M. Sato. Normal state properties and oxygen isotope effect of $(\mathrm{Ba}, \mathrm{K}) \mathrm{BiO}_{3}$. Physica C: Superconductivity, 157(3):469 - 477, 1989.

[49] J. Ranninger B.K. Chakraverty. Bipolarons and superconductivity,. Philosoph. Magaz. B, 52, 1985.

[50] T. M. Rice and L. Sneddon. Real-Space and $\overrightarrow{\mathrm{k}}-$-Space Electron Pairing in $\mathrm{BaPb}_{1-x} \mathrm{Bi}_{x} \mathrm{O}_{3}$. Phys. Rev. Lett., 47:689-692, Aug 1981.

[51] G. Kotliar Z.P. Yin, A. Kutepov. Correlation-enhanced electronphonon coupling: applications of GW and screened hybrid functional to bismuthates, chloronitrides, and other high- $T_{c}$ superconductors. Phys. Rev.X3, 2013.

[52] A. Taraphder, Rahul Pandit, H. R. Krishnamurthy, and T. V. Ramakrishnan. The exotic barium bismuthates. arXiv:cond-mat/9601068v1, 1996.

[53] edited by J. L. Moran-Lopez and L. F. Mattheiss I. K. Schuller. Oxygen Disorder Effects in High- $\mathrm{T}_{c}$ Superconductors. Plenum, New York, p.117, 1990.

[54] Hugh D. Young. Just The Facts 101. Cram101, 2013. 
[55] Patrick M.Woodwards. Chemistry 754 Solid State Chemistry Lecture Notes.

[56] S.F. Lee, J.Y.T. Wei, H.Y. Tang, T.R. Chien, M.K. Wu, and W.Y. Guan. Hall effect and resistivity in metallic $\mathrm{Ba}_{1-x} \mathrm{~K}_{x} \mathrm{BiO}_{3}$ single crystals: Absence of $1 / \mathrm{T}$ dependence in $\mathrm{Rh}$ and linear-to-quadratic evolution of (T). Physica C: Superconductivity, 209(1):141 - 144, 1993.

[57] T. Nishio, H. Minami, , and H. Uwe. Large single crystals of $\mathrm{Ba}_{1-x} \mathrm{~K}_{x} \mathrm{BiO}_{3}$ grown by electrochemical technique. Physica $C$ 35\%360 (2001) 376-379, 2000.

[58] J. S. Swinnea J. P. Wignacourt and et al. Oxygen atom thermal vibration anisotropy in $\mathrm{Ba}_{0.87} \mathrm{~K}_{0.13} \mathrm{BiO}_{3}$. Appl. Phys. Lett., 53, 1753, 1988.

[59] Takashi Noji Yoshinori Imai and et al. Growth of the Single Crystal $\mathrm{BaBiO}_{3}$ by the FloatingZone Method. AIP Conference Proceedings, $850,667,2006$.

[60] R. James Kirkpatrick. Crystal growth from the melt: A review. American Mineralogis, Volume 60, 1975.

[61] Dr. Patrick M. Woodward. Chemistry 754 lecture notes.

[62] CRC Handbook of Chemistry and Physics, 88th Edition. CRC Press, 2007.

[63] D. A. Shirley. High-resolution x-ray photoemission spectrum of the valence bands of gold. Phys. Rev. B, 5:4709-4714, Jun 1972.

[64] Daniel Lubbert. Strain and lattice distortion in semiconductor structures: A synchrotron radiation stud, thesis. Master's thesis, Universitat Potsdam, 1999.

[65] S. Lee, W. Jung, J. Sohn, J. Lee, and S. Cho. Dielectric loss anomaly of $\mathrm{BaBiO}_{3}$. J. Appl. Phys. , 86, 11, 1999.

[66] W. C. Rontgen. Uber eine neue art von strahlen. Sitzungsberichte der Wurzburger Physik-medic. Gesellschaft, 9, 132-141, 1895.

[67] W. C. Rontgen. Eine neue art von strahlen. ii. Sitzungsberichte derWurzburger Physik.-medic. Gesellschaft, 1, 11-19., 1896. 
[68] M. de Broglie. Sur une nouveau procede permettant dobtenir la photographie des spectres de raies des rayons rontgen $\mathrm{m}$. de broglie (1913) sur une nouveau procede permettant dobtenir la photographie des spectres de raies des rayons rontgen,. C. R. Acad. Sci., 157, 924926., 1913.

[69] Farrel W. Lytle. The EXAFS family tree: a personal history of the development of extended $X$-ray absorption ne structure. J. Synchrotron Rad., 1999.

[70] E.A. Stern D.E. Sayers and F.W. Lytle. New technique for investigating noncrystalline structures: Fourier analysis of the extended x-ray absorption fine structure. Phys. Rev. Lett., 27, 1204-1207, 1971.

[71] Carlo Lamberti. X-Ray Absorption and X-Ray Emission Spectroscopy. Swiss Light Source, Paul Scherrer Institute, Switzerland and Institute for Chemical and Bioengineering, ETH Zurich, Switzerland.

[72] Eugen Merzbacher. Quantum Mechanics. John Wiley and Sons, INC., 1961.

[73] Jim Branson. Quantum mechanics course.

[74] J. J. Sakurai. Advanced Quantum Mechanics. Addison-Wesley Publishing Company, 1967.

[75] F. M. F. de Groot. X-ray absorption and dichroism of transition metals and their compounds. J. Electron. Spectrosc. Relat. Phenom., 67(4):529-622, 1994.

[76] E. Z. Kurmaev, R. G. Wilks, A. Moewes, L. D. Finkelstein, S. N. Shamin, and J. Kuneš. Oxygen x-ray emission and absorption spectra as a probe of the electronic structure of strongly correlated oxides. Phys. Rev. B, 77:165127, Apr 2008.

[77] H.A Kramers and W. Z. Heisenberg. ber die streuung von strahlung durch atome. Zeitschrift fr Physik, 1925.

[78] Herman Winick and S. Doniach. Synchrotron Radiation Research. Springer US, 1980. 
[79] M. Merz, N. Ncker, S. Schuppler, D. Arena, J. Dvorak, Y. U. Idzerda, S. N. Ustinovich, A. G. Soldatov, S. V. Shiryaev, and S. N. Barilo. Xray absorption of $\mathrm{Ba}_{1-x} \mathrm{~K}_{x} \mathrm{BiO}_{3}$ and $\mathrm{BaPb}_{y} \mathrm{Bi}_{1-y} \mathrm{O}_{3}$ : Competition between bipolaronic and charge-density wave states. EPL (Europhysics Letters), 72(2):275, 2005.

[80] George A. Sawatzky. Electronic structure of correlated electron systems-lecture notes.

[81] M. T. Czyżyk and G. A. Sawatzky. Local-density functional and onsite correlations: The electronic structure of $\mathrm{La}_{2} \mathrm{CuO}_{4}$ and $\mathrm{LaCuO}_{3}$. Phys. Rev. B, 49:14211-14228, May 1994.

[82] A. Einstein. ber einen die erzeugung und verwandlung des lichtes betreffenden heuristischen gesichtspunkt. Ann. Phys. 17, 1905.

[83] C.S. Fadley. X-ray photoelectron spectroscopy: Progress and perspectives. Journal of Electron Spectroscopy and Related Phenomena, 178(Supplement C):2 - 32, 2010. Trends in X-ray Photoelectron Spectroscopy of solids (theory, techniques and applications).

[84] Lars Hedin. New method for calculating the one-particle green's function with application to the electron-gas problem. Phys. Rev., 139:A796-A823, Aug 1965.

[85] C. J. Ballhausen. Introduction to Ligand Field Theory. McGraw-Hill Book Co, 1962.

[86] R. D. Cowan. The Theory of Atomic Structure and Spectra. University of California Press, 1981.

[87] Attila Szabu and Neil S. Ostlund. Modern Quantum Chemistry: Introduction to Advanced Electronic Structure Theory. Dover Publications, 1996.

[88] C.E. Moore. Atomic energy levels. NBS Circular, (467), 1949.

[89] J. Becquerel. Z phyzik 58:205, 1929.

[90] H. Bethe. Ann phyzik, [5] 3:135, 1929.

[91] J. H. Van Vleck. Theory of the variations in paramagnetic anisotropy among different salts of the iron group. Phys. Rev., 41:208-215, 1932. 
[92] Shadi Balandeh. X-ray absorption and resonant inelastic x-ray scattering calculations with ligand field single cluster method on praseodymium nickle oxide. Master's thesis, The University of British Columbia, 2013.

[93] G.L. Miessler and D.A. Tarr. Inorganic Chemistry. Prentice-Hall, 1999.

[94] Dick van der Marel. The electronic structure of embedded transition metal atoms. $\mathrm{PhD}$ thesis, University of Groningen, 1985.

[95] D. van der Marel and G. A. Sawatzky. Electron-electron interaction and localization in $\mathrm{d}$ and $\mathrm{f}$ transition metals. Phys. Rev. B, 37:1067410684, Jun 1988.

[96] J. S. Griffith and L. E. Orgel. Ligand-field theory. Q. Rev. Chem. Soc., 11:381-393, 1957.

[97] C. Lanczos. An iteration method for the solution of the eigenvalue problem of linear differential and integral operators. J. Res. Nat. Bur. Stand 45(4):255-282, 1950.

[98] W. Maurits Haverkort. Spin and orbital degrees of freedom in transition metal oxides and oxide thin flms studied by soft x-ray absorption spectroscopy. PhD thesis, University of Cologne, 2005.

[99] M. W. Haverkort. Quanty - a quantum many body script language: http://www.quanty.org, 2015.

[100] M. W. Haverkort, G. Sangiovanni, P. Hansmann, A. Toschi, Y. Lu, and S. Macke. Bands, resonances, edge singularities and excitons in core level spectroscopy investigated within the dynamical mean-field theory. Euro. Phys. Lett. 108, 57004, 2014.

[101] Haverkort M. W., Zwierzycki, M., and Andersen O. K. Multiplet ligand-field theory using wannier orbitals. Phys. Rev. B, 85:165113, Apr 2012.

[102] J. C. Slater and G. F. Koster. Simplified lcao method for the periodic potential problem. Phys. Rev. 94, 1954.

[103] A. Khazraie, K. Foyevtsova, I. Elfimov, and G. A. Sawatzky. To be published. 
[104] I. S. Elfimov, S. Yunoki, and G. A. Sawatzky. Possible path to a new class of ferromagnetic and half-metallic ferromagnetic materials. Phys. Rev. Lett., 89:216403, Nov 2002.

[105] Zaanen J., Westra C., and Sawatzky G. A. Determination of the electronic structure of transition-metal compounds: $2 \mathrm{p}$ x-ray photoemission spectroscopy of the nickel dihalides. Phys. Rev. B, 33:8060-8073, Jun 1986.

[106] Green R. J., Haverkort M. W., and Sawatzky G. A. Bond disproportionation and dynamical charge fluctuations in the perovskite rareearth nickelates. Phys. Rev. B, 94:195127, Nov 2016.

[107] A. E. Bocquet, A. Fujimori, T. Mizokawa, T. Saitoh, H. Namatame, S. Suga, N. Kimizuka, Y. Takeda, and M. Takano. Electronic structure of $\mathrm{SrFe}^{4+} \mathrm{O}_{3}$ and related Fe perovskite oxides. Phys. Rev. B, 45:15611570, Jan 1992.

[108] A. Khazraie, I. Elfimov, K. Foyevtsova, and to be published G. A. Sawatzky. Electron doping in the bismuthates via adatom. To be Published.

[109] N. C. Plumb, D. J. Gawryluk, Y. Wang, Z. Ristić, J. Park, B. Q. Lv, Z. Wang, C. E. Matt, N. Xu, T. Shang, K. Conder, J. Mesot, S. Johnston, M. Shi, and M. Radović. Momentum-Resolved Electronic Structure of the High- $T_{c}$ Superconductor Parent Compound $\mathrm{BaBiO}_{3}$. Phys. Rev. Lett., 117:037002, Jul 2016. 


\section{Appendix A \\ $\mathrm{BaBiO}_{3}$ Bragg Reflections}


Appendix A. $\mathrm{BaBiO}_{3}$ Bragg Reflections

\begin{tabular}{|c|c|c|c|c|c|c|c|c|c|c|c|}
\hline h & k & 1 & $d(\AA)$ & $F($ real) & $F$ (imag) & $|F|$ & $2 \theta$ & $\sim 1$ & M & $\lambda$ & Phase \\
\hline 0 & 1 & 1 & 5.02426 & 0.164726 & 0.658186 & 0.678486 & 17.6813 & 0.0003 & 4 & 2 & 1 \\
\hline 1 & 1 & 0 & 4.36845 & 121.475 & 1.67427 & 121.487 & 20.3123 & 16.7710 & 4 & 1 & 1 \\
\hline 1 & 1 & 0 & 4.36845 & 121.56 & 1.67592 & 121.572 & 20.362 & 8.3548 & 4 & 2 & 1 \\
\hline 0 & 0 & 2 & 4.34739 & 118.742 & 1.67529 & 118.754 & 20.4118 & 7.9318 & 2 & 1 & 1 \\
\hline 0 & 0 & 2 & 4.34739 & 118.826 & 1.67695 & 118.838 & 20.4618 & 3.9515 & 2 & 2 & 1 \\
\hline 2 & 0 & 0 & 3.09999 & 417.031 & 67.1436 & 422.402 & 28.7756 & 48.8065 & 2 & 1 & 1 \\
\hline 2 & 0 & 0 & 3.09999 & 416.984 & 67.3871 & 422.394 & 28.8468 & 24.2740 & 2 & 2 & 1 \\
\hline 1 & 1 & -2 & 3.08433 & 424.123 & 67.1406 & 429.404 & 28.9248 & 99.7689 & 4 & 1 & 1 \\
\hline 1 & 1 & 2 & 3.07865 & 425.491 & 67.2147 & 430.767 & 28.9794 & 100.0000 & 4 & 1 & 1 \\
\hline 0 & 2 & 0 & 3.07805 & 422.946 & 67.2035 & 428.252 & 28.9852 & 49.3968 & 2 & 1 & 1 \\
\hline 1 & 1 & -2 & 3.08433 & 424.076 & 67.3841 & 429.396 & 28.9964 & 49.6200 & 4 & 2 & 1 \\
\hline 1 & 1 & 2 & 3.07865 & 425.443 & 67.4585 & 430.758 & 29.0511 & 49.7348 & 4 & 2 & 1 \\
\hline 0 & 2 & 0 & 3.07805 & 422.898 & 67.4473 & 428.243 & 29.0569 & 24.5674 & 2 & 2 & 1 \\
\hline 2 & 1 & -1 & 2.64001 & 28.7805 & 2.58231 & 28.8961 & 33.9289 & 0.3204 & 4 & 1 & 1 \\
\hline 2 & 1 & 1 & 2.63644 & -21.3101 & -1.2703 & 21.3479 & 33.9762 & 0.1743 & 4 & 1 & 1 \\
\hline 2 & 1 & -1 & 2.64001 & 28.7761 & 2.59194 & 28.8926 & 34.0135 & 0.1593 & 4 & 2 & 1 \\
\hline 2 & 1 & 1 & 2.63644 & -21.3083 & -1.27508 & 21.3464 & 34.061 & 0.0867 & 4 & 2 & 1 \\
\hline 1 & 2 & -1 & 2.62891 & 11.1513 & -1.46941 & 11.2477 & 34.0764 & 0.0481 & 4 & 1 & 1 \\
\hline 1 & 0 & -3 & 2.62819 & -17.9509 & 2.70166 & 18.1531 & 34.0861 & 0.0626 & 2 & 1 & 1 \\
\hline 1 & 2 & 1 & 2.62715 & -14.5643 & 0.178477 & 14.5654 & 34.1 & 0.0805 & 4 & 1 & 1 \\
\hline 1 & 0 & 3 & 2.62292 & 41.6332 & 1.24283 & 41.6517 & 34.1567 & 0.3280 & 2 & 1 & 1 \\
\hline 1 & 2 & -1 & 2.62891 & 11.1551 & -1.47474 & 11.2521 & 34.1615 & 0.0239 & 4 & 2 & 1 \\
\hline 0 & 1 & 3 & 2.62219 & -6.85943 & -1.94529 & 7.12993 & 34.1665 & 0.0192 & 4 & 1 & 1 \\
\hline 1 & 0 & -3 & 2.62819 & -17.9576 & 2.71147 & 18.1612 & 34.1712 & 0.0312 & 2 & 2 & 1 \\
\hline 1 & 2 & 1 & 2.62715 & -14.5653 & 0.179052 & 14.5664 & 34.1851 & 0.0400 & 4 & 2 & 1 \\
\hline 1 & 0 & 3 & 2.62292 & 41.6323 & 1.24762 & 41.651 & 34.242 & 0.1631 & 2 & 2 & 1 \\
\hline 0 & 1 & 3 & 2.62219 & -6.85545 & -1.95247 & 7.12806 & 34.2518 & 0.0095 & 4 & 2 & 1 \\
\hline 2 & 0 & -2 & 2.52715 & 32.9622 & 1.34635 & 32.9897 & 35.4933 & 0.1892 & 2 & 1 & 1 \\
\hline 2 & 0 & -2 & 2.52715 & 33.042 & 1.3465 & 33.0695 & 35.5822 & 0.0946 & 2 & 2 & 1 \\
\hline 2 & 0 & 2 & 2.52090 & 31.2698 & 1.19301 & 31.2925 & 35.5842 & 0.1693 & 2 & 1 & 1 \\
\hline 2 & 0 & 2 & 2.52090 & 31.3498 & 1.19259 & 31.3725 & 35.6733 & 0.0846 & 2 & 2 & 1 \\
\hline 0 & 2 & 2 & 2.51213 & 25.6682 & 1.18204 & 25.6954 & 35.7126 & 0.2265 & 4 & 1 & 1 \\
\hline 0 & 2 & 2 & 2.51213 & 25.7481 & 1.18155 & 25.7751 & 35.802 & 0.1133 & 4 & 2 & 1 \\
\hline 2 & 2 & 0 & 2.18423 & 451.886 & 65.8763 & 456.663 & 41.3006 & 51.8701 & 4 & 1 & 1 \\
\hline 2 & 2 & 0 & 2.18423 & 451.842 & 66.1157 & 456.654 & 41.4052 & 25.7879 & 4 & 2 & 1 \\
\hline 0 & 0 & 4 & 2.17369 & 442.732 & 65.7415 & 447.586 & 41.51 & 24.6344 & 2 & 1 & 1 \\
\hline 0 & 0 & 4 & 2.17369 & 442.688 & 65.9804 & 447.578 & 41.6151 & 12.2473 & 2 & 2 & 1 \\
\hline 3 & 0 & -1 & 2.01183 & 15.2774 & -3.12166 & 15.593 & 45.0249 & 0.0249 & 2 & 1 & 1 \\
\hline 3 & 0 & 1 & 2.00946 & -7.2098 & 1.94175 & 7.4667 & 45.0808 & 0.0057 & 2 & 1 & 1 \\
\hline 3 & 0 & -1 & 2.01183 & 15.2851 & -3.133 & 15.6029 & 45.1399 & 0.0124 & 2 & 2 & 1 \\
\hline 3 & 0 & 1 & 2.00946 & -7.21451 & 1.94882 & 7.47309 & 45.1961 & 0.0028 & 2 & 2 & 1 \\
\hline 2 & 1 & -3 & 2.00437 & -26.4095 & -3.7593 & 26.6758 & 45.2018 & 0.1445 & 4 & 1 & 1 \\
\hline 2 & 1 & 3 & 1.99969 & 15.4488 & -0.0138725 & 15.4488 & 45.3133 & 0.0482 & 4 & 1 & 1 \\
\hline
\end{tabular}

Figure A.1: The constructive Bragg reflections for monoclinic $\mathrm{BaBiO}_{3}$ with lattice parameters given in table 2.1 for $17.68^{\circ} \leq 2 \theta \leq 63.29^{\circ}$ calculated via Vesta program. To be continued on the next two pages. 
Appendix A. $\mathrm{BaBiO}_{3}$ Bragg Reflections

\begin{tabular}{|c|c|c|c|c|c|c|c|c|c|c|c|}
\hline $\mathrm{h}$ & $k$ & I & $d(\AA)$ & F(real) & F(imag) & $|\mathrm{F}|$ & $2 \theta$ & & M & $\lambda$ & Phase \\
\hline 2 & 1 & -3 & 2.00437 & -26.4095 & -3.7593 & 26.6758 & 45.2018 & 0.1445 & 4 & 1 & 1 \\
\hline 2 & 1 & 3 & 1.99969 & 15.4488 & -0.0138725 & 15.4488 & 45.3133 & 0.0482 & 4 & 1 & 1 \\
\hline 2 & 1 & -3 & 2.00437 & -26.4026 & -3.77325 & 26.6708 & 45.3173 & 0.0718 & 4 & 2 & 1 \\
\hline 0 & 3 & 1 & 1.99717 & 5.28586 & 0.652812 & 5.32602 & 45.3738 & 0.0057 & 4 & 1 & 1 \\
\hline 1 & 2 & 3 & 1.99640 & -4.39906 & 0.955487 & 4.50163 & 45.3922 & 0.0041 & 4 & 1 & 1 \\
\hline 2 & 1 & 3 & 1.99969 & 15.4497 & -0.0138217 & 15.4497 & 45.4292 & 0.0240 & 4 & 2 & 1 \\
\hline 0 & 3 & 1 & 1.99717 & 5.28469 & 0.655239 & 5.32515 & 45.4898 & 0.0028 & 4 & 2 & 1 \\
\hline 1 & 2 & 3 & 1.99640 & -4.40143 & 0.95896 & 4.50468 & 45.5083 & 0.0020 & 4 & 2 & 1 \\
\hline 3 & 1 & 0 & 1.95920 & 88.9897 & 1.62707 & 89.0046 & 46.3035 & 1.5235 & 4 & 1 & 1 \\
\hline 3 & 1 & 0 & 1.95920 & 89.0696 & 1.62872 & 89.0845 & 46.4222 & 0.7587 & 4 & 2 & 1 \\
\hline 2 & 2 & -2 & 1.95318 & 97.4635 & 1.71733 & 97.4787 & 46.4547 & 1.8140 & 4 & 1 & 1 \\
\hline 2 & 2 & 2 & 1.95029 & 104.14 & 1.62323 & 104.153 & 46.5275 & 2.0636 & 4 & 1 & 1 \\
\hline 2 & 2 & 2 & 1.95029 & 104.221 & 1.62498 & 104.234 & 46.6469 & 1.0274 & 4 & 2 & 1 \\
\hline 1 & 1 & 4 & 1.94465 & 94.525 & 1.59617 & 94.5385 & 46.6704 & 1.6884 & 4 & 1 & 1 \\
\hline 1 & 3 & 0 & 1.94810 & 97.4681 & 1.58754 & 97.481 & 46.7024 & 0.8962 & 4 & 2 & 1 \\
\hline 1 & 1 & -4 & 1.94752 & 100.601 & 1.77791 & 100.617 & 46.7173 & 0.9541 & 4 & 2 & 1 \\
\hline 1 & 1 & 4 & 1.94465 & 94.6052 & 1.59776 & 94.6187 & 46.7902 & 0.8408 & 4 & 2 & 1 \\
\hline 3 & 1 & -2 & 1.78786 & 352.482 & 63.6152 & 358.176 & 51.0428 & 19.7728 & 4 & 1 & 1 \\
\hline 3 & 1 & 2 & 1.78454 & 357.962 & 63.8447 & 363.611 & 51.1446 & 20.2851 & 4 & 1 & 1 \\
\hline 3 & 1 & -2 & 1.78786 & 352.437 & 63.846 & 358.174 & 51.1753 & 9.8280 & 4 & 2 & 1 \\
\hline 2 & 0 & -4 & 1.78195 & 357.323 & 63.5748 & 362.935 & 51.2242 & 10.0692 & 2 & 1 & 1 \\
\hline 3 & 1 & 2 & 1.78454 & 357.917 & 64.0763 & 363.607 & 51.2775 & 10.0826 & 4 & 2 & 1 \\
\hline 1 & 3 & -2 & 1.77832 & 344.387 & 63.6746 & 350.224 & 51.3365 & 18.6591 & 4 & 1 & 1 \\
\hline 3 & 0 & -3 & 1.68476 & 30.4014 & 4.55488 & 30.7407 & 54.4149 & 0.0630 & 2 & 1 & 1 \\
\hline 3 & 2 & -1 & 1.68403 & -13.4911 & -3.23284 & 13.873 & 54.4407 & 0.0256 & 4 & 1 & 1 \\
\hline 3 & 2 & 1 & 1.68264 & -5.49384 & 1.88971 & 5.80975 & 54.4894 & 0.0045 & 4 & 1 & 1 \\
\hline 3 & 0 & -3 & 1.68476 & 30.393 & 4.57179 & 30.7349 & 54.5576 & 0.0313 & 2 & 2 & 1 \\
\hline 3 & 0 & 3 & 1.68060 & -0.848225 & -0.738303 & 1.12453 & 54.5609 & 0.0001 & 2 & 1 & 1 \\
\hline 3 & 2 & -1 & 1.68403 & -13.4846 & -3.24478 & 13.8695 & 54.5835 & 0.0127 & 4 & 2 & 1 \\
\hline 2 & 3 & -1 & 1.67937 & 17.4183 & 2.42082 & 17.5857 & 54.6042 & 0.0409 & 4 & 1 & 1 \\
\hline
\end{tabular}


Appendix A. $\mathrm{BaBiO}_{3}$ Bragg Reflections

\begin{tabular}{|c|c|c|c|c|c|c|c|c|c|c|c|}
\hline h & k & I & $d(\AA)$ & $F($ real) & $F$ (imag) & $|F|$ & $2 \theta$ & 1 & M & $\lambda$ & Phase \\
\hline 3 & 2 & 1 & 1.68264 & -5.49843 & 1.89659 & 5.81634 & 54.6324 & 0.0022 & 4 & 2 & 1 \\
\hline 2 & 3 & 1 & 1.67845 & -19.1814 & -1.23555 & 19.2212 & 54.6366 & 0.0488 & 4 & 1 & 1 \\
\hline 3 & 0 & 3 & 1.68060 & -0.846616 & -0.741014 & 1.1251 & 54.7041 & 0.0000 & 2 & 2 & 1 \\
\hline 1 & 0 & -5 & 1.67548 & -44.3564 & -4.17604 & 44.5526 & 54.7414 & 0.1304 & 2 & 1 & 1 \\
\hline 2 & 3 & -1 & 1.67937 & 17.4139 & 2.42982 & 17.5826 & 54.7475 & 0.0203 & 4 & 2 & 1 \\
\hline 0 & 3 & 3 & 1.67475 & -2.7751 & -1.82055 & 3.31897 & 54.7672 & 0.0014 & 4 & 1 & 1 \\
\hline 2 & 3 & 1 & 1.67845 & -19.1799 & -1.24023 & 19.2199 & 54.78 & 0.0242 & 4 & 2 & 1 \\
\hline 0 & 1 & 5 & 1.67347 & 8.18435 & 3.05808 & 8.73702 & 54.8128 & 0.0100 & 4 & 1 & 1 \\
\hline 1 & 0 & 5 & 1.67320 & 5.3148 & -2.11324 & 5.71952 & 54.8222 & 0.0021 & 2 & 1 & 1 \\
\hline 1 & 0 & -5 & 1.67548 & -44.3498 & -4.19168 & 44.5475 & 54.8851 & 0.0648 & 2 & 2 & 1 \\
\hline 0 & 3 & 3 & 1.67475 & -2.77117 & -1.82724 & 3.31937 & 54.911 & 0.0007 & 4 & 2 & 1 \\
\hline 0 & 1 & 5 & 1.67347 & 8.17798 & 3.06934 & 8.73501 & 54.9567 & 0.0050 & 4 & 2 & 1 \\
\hline 1 & 0 & 5 & 1.67320 & 5.31989 & -2.12094 & 5.7271 & 54.9662 & 0.0011 & 2 & 2 & 1 \\
\hline 4 & 0 & 0 & 1.54999 & 360.659 & 62.3297 & 366.006 & 59.5991 & 7.2639 & 2 & 1 & 1 \\
\hline 4 & 0 & 0 & 1.54999 & 360.618 & 62.5561 & 366.003 & 59.7581 & 3.6103 & 2 & 2 & 1 \\
\hline 2 & 2 & -4 & 1.54217 & 359.312 & 62.1755 & 364.652 & 59.9325 & 14.2414 & 4 & 1 & 1 \\
\hline 2 & 2 & 4 & 1.53932 & 360.745 & 62.4333 & 366.108 & 60.0545 & 14.2902 & 4 & 1 & 1 \\
\hline 0 & 4 & 0 & 1.53902 & 373.645 & 62.5477 & 378.844 & 60.0674 & 7.6472 & 2 & 1 & 1 \\
\hline 2 & 2 & -4 & 1.54217 & 359.27 & 62.4013 & 364.649 & 60.0926 & 7.0782 & 4 & 2 & 1 \\
\hline 2 & 2 & 4 & 1.53932 & 360.703 & 62.6601 & 366.105 & 60.215 & 7.1025 & 4 & 2 & 1 \\
\hline 0 & 4 & 0 & 1.53902 & 373.604 & 62.7751 & 378.841 & 60.2279 & 3.8008 & 2 & 2 & 1 \\
\hline 4 & 1 & -1 & 1.48175 & 29.0551 & 4.11076 & 29.3445 & 62.6453 & 0.0836 & 4 & 1 & 1 \\
\hline 4 & 1 & 1 & 1.48048 & -25.6844 & -2.92058 & 25.8499 & 62.7048 & 0.0647 & 4 & 1 & 1 \\
\hline 4 & 1 & -1 & 1.48175 & 29.0479 & 4.12606 & 29.3394 & 62.8143 & 0.0415 & 4 & 2 & 1 \\
\hline 3 & 2 & -3 & 1.47787 & -11.7315 & 4.09528 & 12.4258 & 62.8284 & 0.0149 & 4 & 1 & 1 \\
\hline 4 & 1 & 1 & 1.48048 & -25.6796 & -2.9315 & 25.8464 & 62.874 & 0.0322 & 4 & 2 & 1 \\
\hline 3 & 2 & 3 & 1.47506 & 20.1395 & -0.534301 & 20.1466 & 62.9619 & 0.0390 & 4 & 1 & 1 \\
\hline 2 & 3 & -3 & 1.47441 & -23.3905 & -3.58743 & 23.664 & 62.9928 & 0.0537 & 4 & 1 & 1 \\
\hline 3 & 2 & -3 & 1.47787 & -11.7417 & 4.11017 & 12.4403 & 62.998 & 0.0074 & 4 & 2 & 1 \\
\hline 2 & 1 & -5 & 1.47415 & 25.3316 & 4.75977 & 25.7749 & 63.0049 & 0.0637 & 4 & 1 & 1 \\
\hline 2 & 3 & 3 & 1.47254 & 7.95256 & -0.039507 & 7.95266 & 63.0817 & 0.0060 & 4 & 1 & 1 \\
\hline 1 & 4 & -1 & 1.47228 & -9.24787 & -1.48356 & 9.36612 & 63.0941 & 0.0084 & 4 & 1 & 1 \\
\hline 1 & 4 & 1 & 1.47197 & -1.02823 & 0.2278 & 1.05316 & 63.1089 & 0.0001 & 4 & 1 & 1 \\
\hline 1 & 2 & -5 & 1.47159 & 7.71462 & -3.65642 & 8.53726 & 63.1271 & 0.0070 & 4 & 1 & 1 \\
\hline 3 & 2 & 3 & 1.47506 & 20.1422 & -0.53608 & 20.1493 & 63.132 & 0.0194 & 4 & 2 & 1 \\
\hline 2 & 1 & 5 & 1.47105 & -5.51568 & 1.22618 & 5.65033 & 63.153 & 0.0030 & 4 & 1 & 1 \\
\hline 2 & 3 & -3 & 1.47441 & -23.384 & -3.60077 & 23.6596 & 63.1629 & 0.0267 & 4 & 2 & 1 \\
\hline 2 & 1 & -5 & 1.47415 & 25.3226 & 4.77741 & 25.7693 & 63.1751 & 0.0316 & 4 & 2 & 1 \\
\hline 1 & 2 & 5 & 1.47005 & -39.1169 & -2.43672 & 39.1927 & 63.2011 & 0.1462 & 4 & 1 & 1 \\
\hline 2 & 3 & 3 & 1.47254 & 7.95322 & -0.0395817 & 7.95332 & 63.2521 & 0.0030 & 4 & 2 & 1 \\
\hline 1 & 4 & -1 & 1.47228 & -9.24517 & -1.48907 & 9.36432 & 63.2646 & 0.0042 & 4 & 2 & 1 \\
\hline 1 & 4 & 1 & 1.47197 & -1.02882 & 0.228624 & 1.05392 & 63.2794 & 0.0001 & 4 & 2 & 1 \\
\hline 1 & 2 & -5 & 1.47159 & 7.72348 & -3.66974 & 8.55097 & 63.2977 & 0.0035 & 4 & 2 & 1 \\
\hline
\end{tabular}

